Effect of a 6-week Strengthening Program on Shoulder and Scapular Stabilizer Strength and Scapular Kinematics in Division I Collegiate Swimmers

\author{
Elizabeth Eve Hibberd
}

A thesis submitted to the faculty of the University of North Carolina at Chapel Hill in partial fulfillment of the requirements for the degree of Master of Arts in the Department of Exercise \& Sport Science in the College of Arts \& Sciences.

Chapel Hill

2010

Approved by:

Advisor: Joseph Myers, PhD, ATC

Reader: William Prentice, PhD, ATC

Reader: Sakiko Oyama, MS, ATC

Reader: Jeffrey Spang, MD 
(C) 2010

Elizabeth Eve Hibberd

ALL RIGHTS RESERVED 


\begin{abstract}
Elizabeth Eve Hibberd: Effect of a 6-week Strengthening Program on Scapular Stabilizer Strength and Scapular Kinematics in Division I Collegiate Swimmers (Under the direction of Dr. Joseph Myers, Dr. William Prentice, Dr. Jeffrey Spang, and Sakiko Oyama)

Shoulder injuries are common in swimmers because of the demands of the sport. Muscle imbalances frequently exist due to the biomechanics of the sport, which predispose swimmers to injury. The objective of this study was to assess the effectiveness of a 6week intervention program to improve shoulder and scapular stabilizer strength and scapular kinematics in collegiate swimmers. Shoulder and scapular stabilizer strength was measured with a handheld dynamometer and scapular kinematics were assessed using an electromagnetic tracking device. This study found that there were trends towards significance in shoulder flexion and abduction with greater improvement occurring in the intervention group. Scapular kinematic data revealed increased scapular internal rotation, protraction, and elevation in all subjects at post testing, but there was not a significant effect of group on the individual kinematic variables. There was no significant effect of group on subjective rating of the shoulder.
\end{abstract}




\section{Table of Contents}

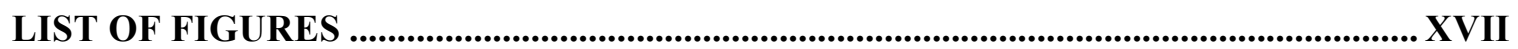

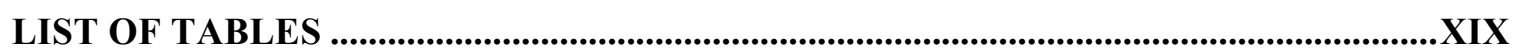

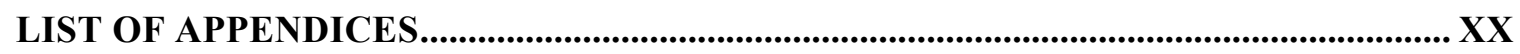

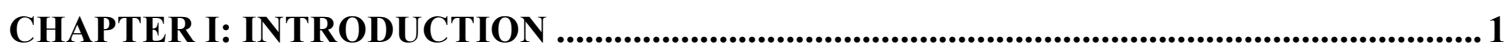

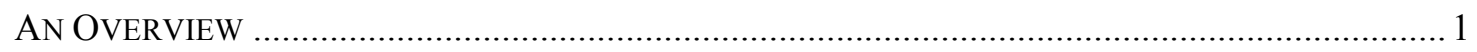

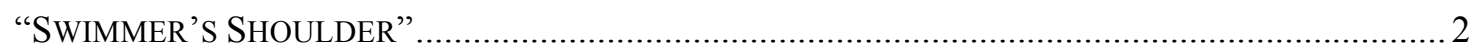

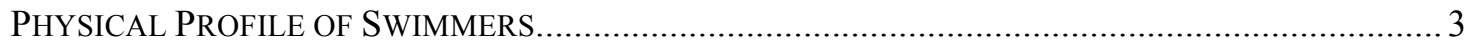

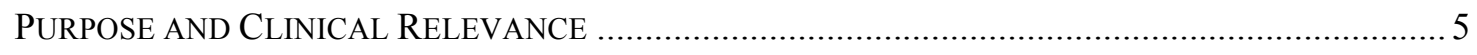

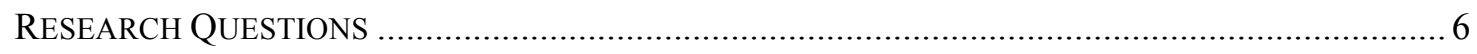

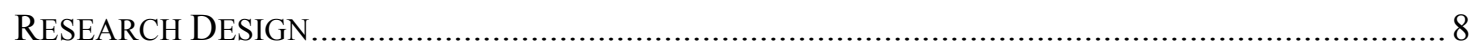

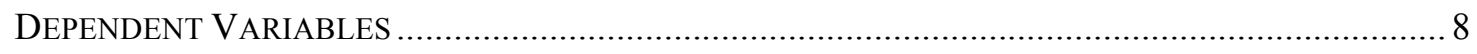

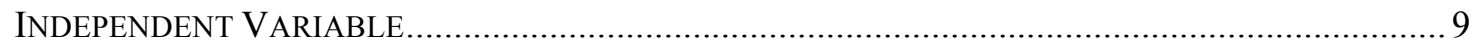

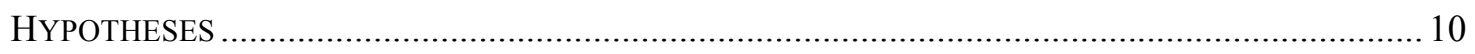

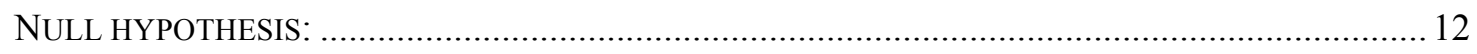

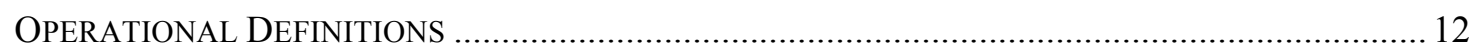

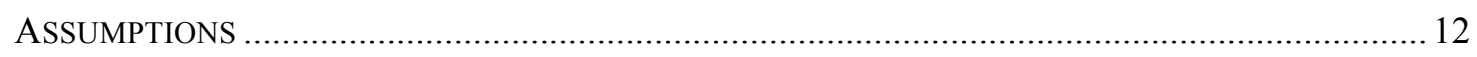

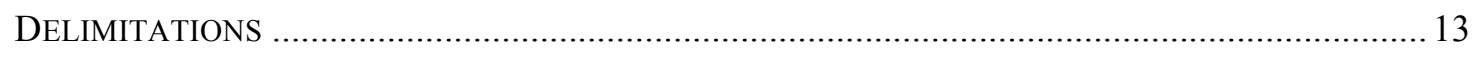

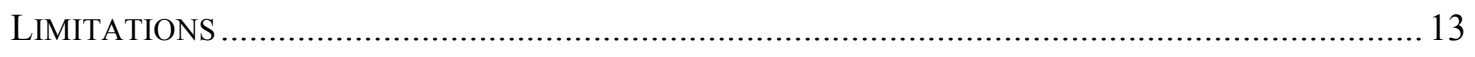




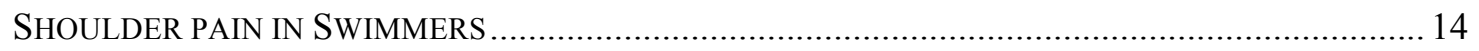

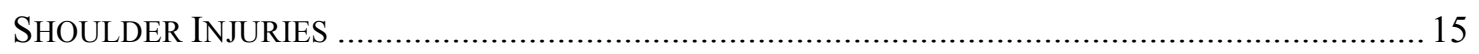

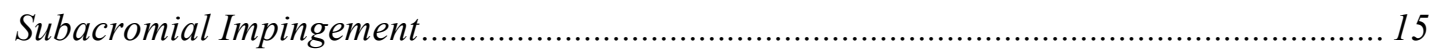

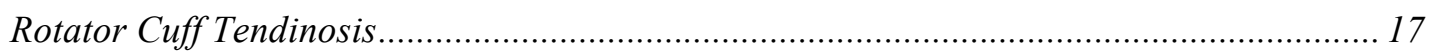

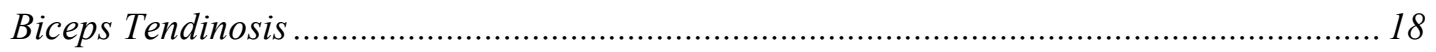

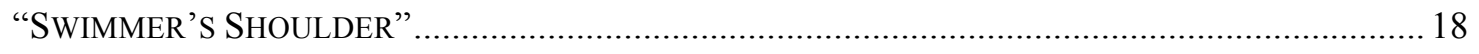

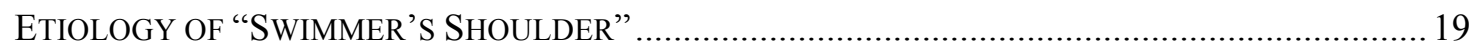

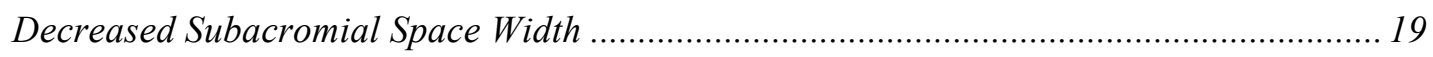

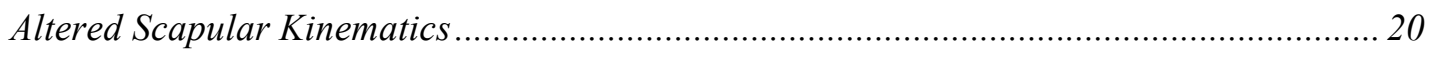

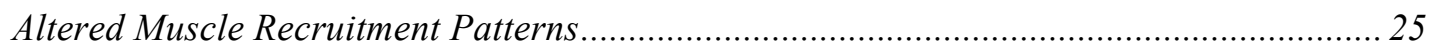

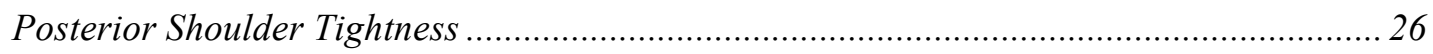

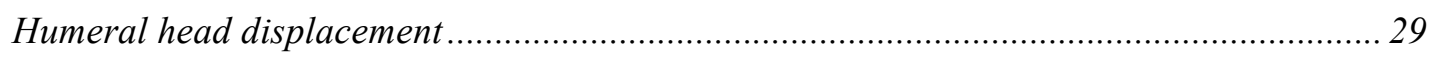

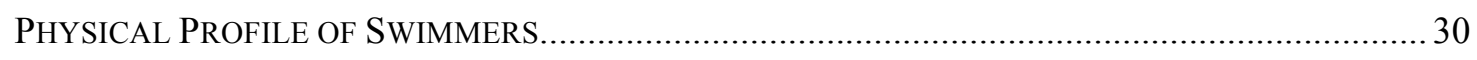

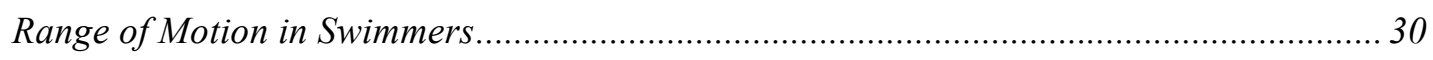

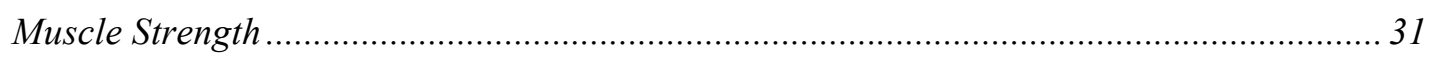

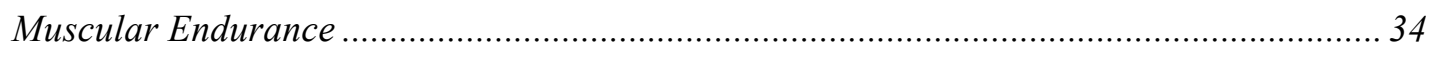

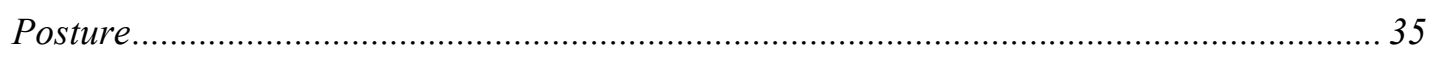

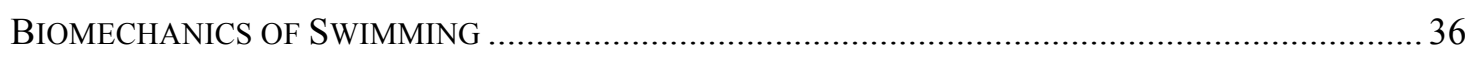

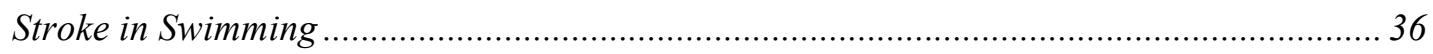

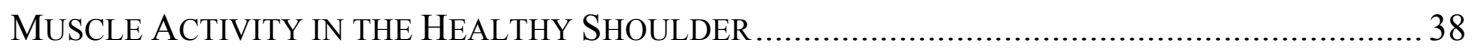

MUSCLE ACTIVITY IN THE PAINFUL SHOULDER ............................................................ 40

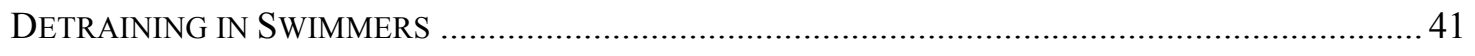

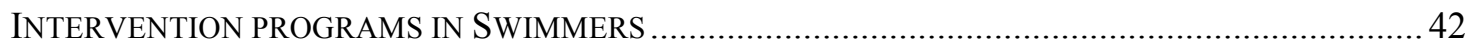

EMG ANALYSIS OF SHOULDER STRENGTHENING EXERCISES ........................................ 45 


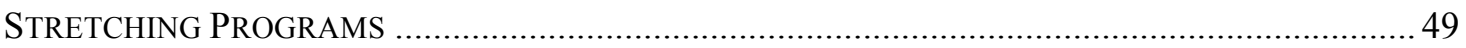

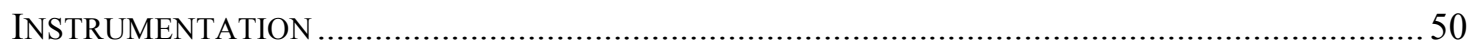

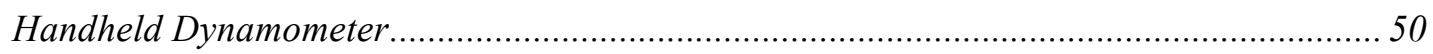

Motion Monitor electromagnetic tracking device ........................................................... 50

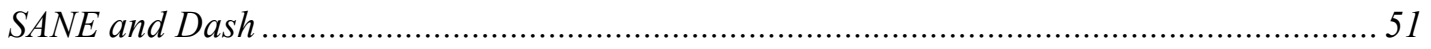

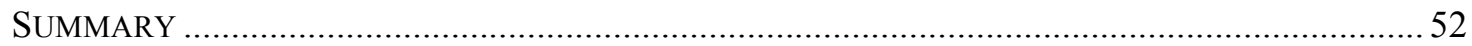

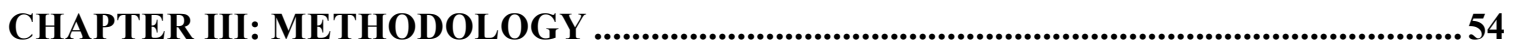

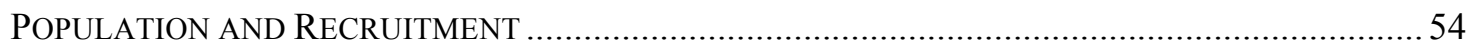

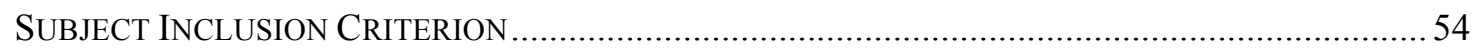

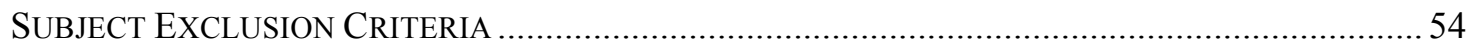

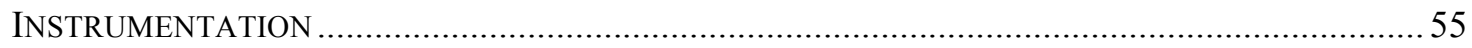

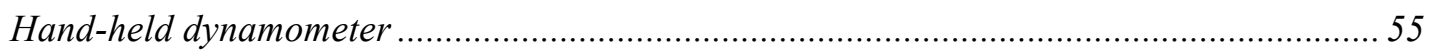

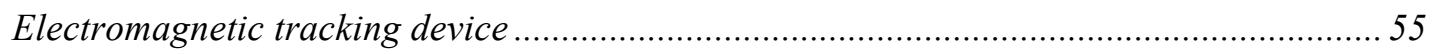

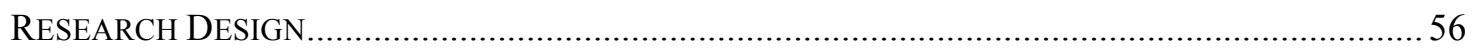

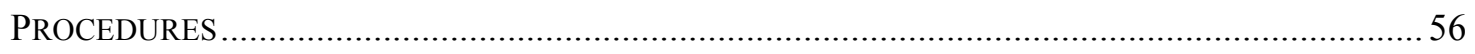

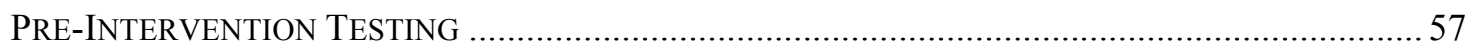

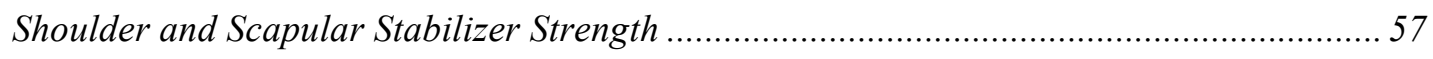

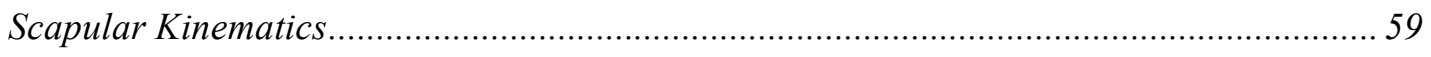

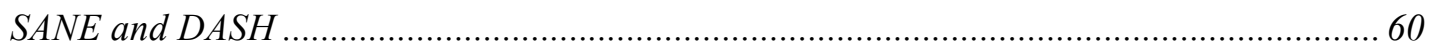

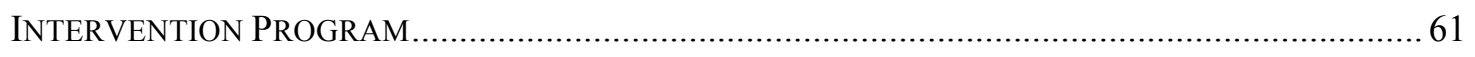

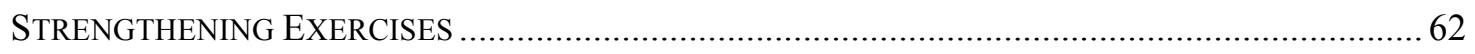

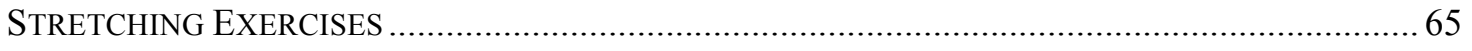

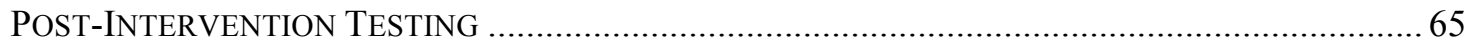

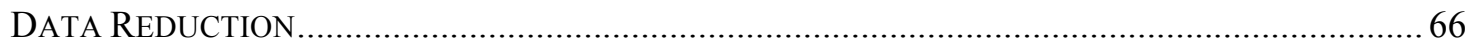

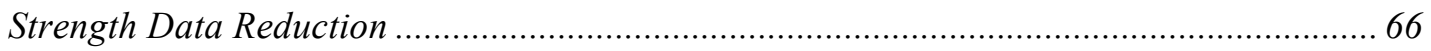




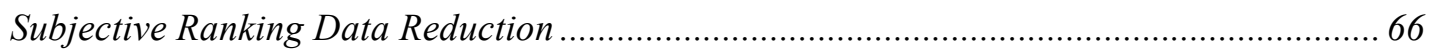

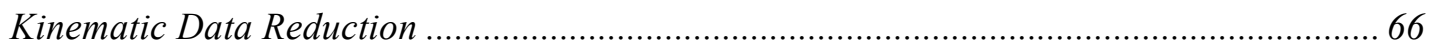

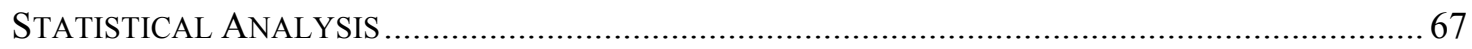

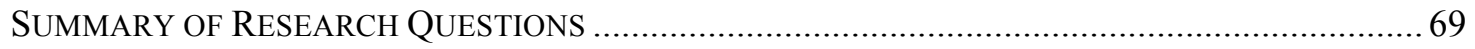

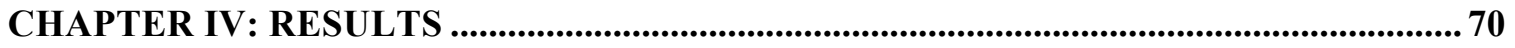

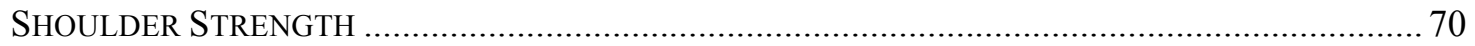

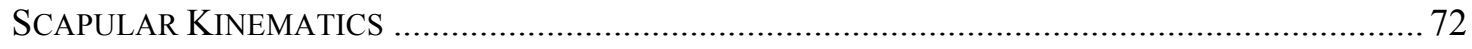

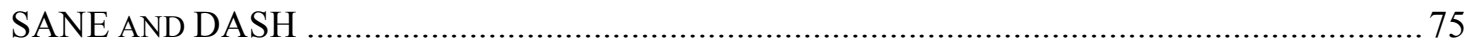

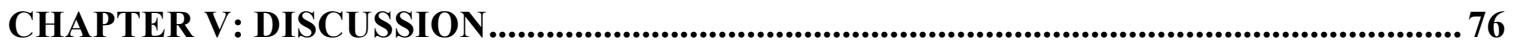

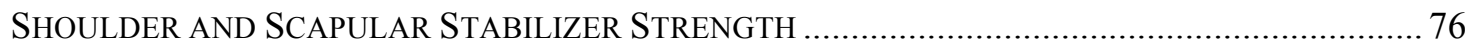

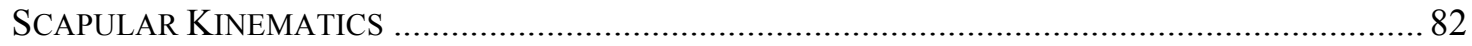

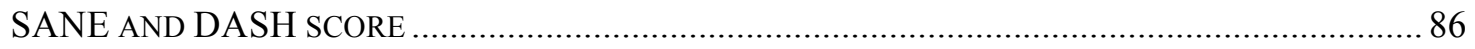

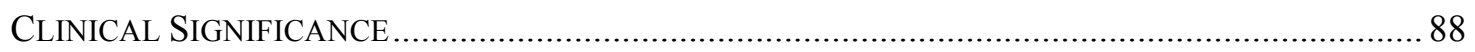

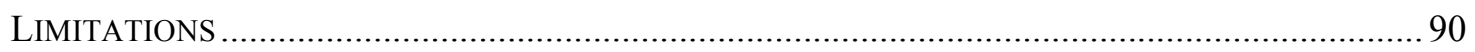

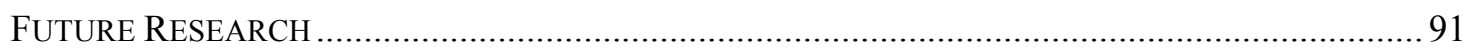

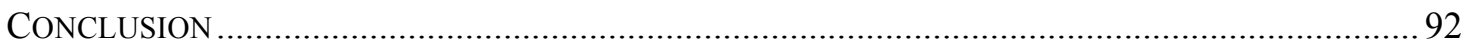

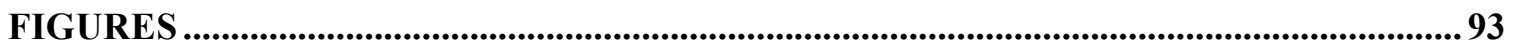

TABLES

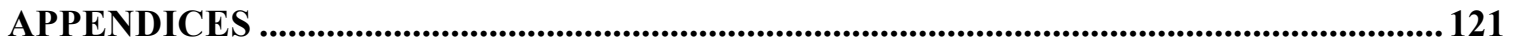

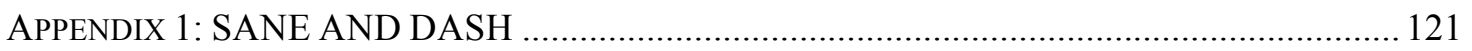

APPENDIX 2: MANUSCRIPT FORMATTED FOR CLINICAL JOURNAL OF SPORTS MEDICINE ...... 122

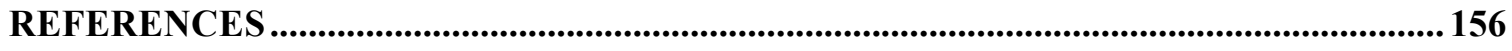




\section{List of Figures}

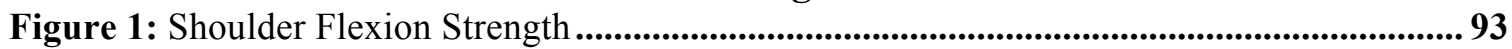

Figure 2: Shoulder Extension Strength .................................................................................................. 93

Figure 3: Shoulder External Rotation Strength ...........................................................................94

Figure 4: Shoulder Internal Rotation Strength .................................................................................. 94

Figure 5: Shoulder Abduction Strength ..................................................................................................... 94

Figure 6: Shoulder Adduction Strength ..................................................................................................... 95

Figure 7: Scapular Retraction Strength ............................................................................................ 95

Figure 8: Scapular Retraction with Upward Rotation Strength ........................................................ 95

Figure 9: Scapular Retraction with Downward Rotation Strength ...............................................96

Figure 10: Assessment of Scapular Kinematics.............................................................................96

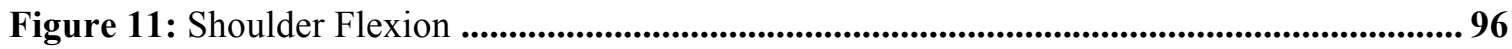

Figure 12: Shoulder Extension..................................................................................................................... 97

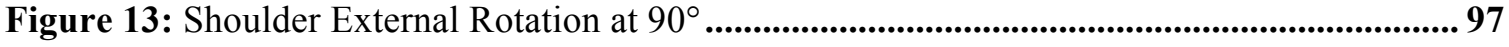

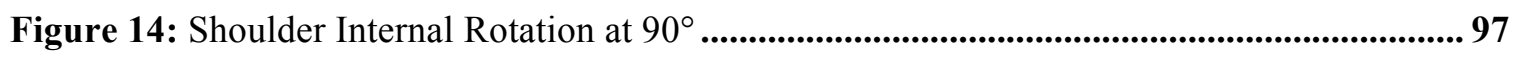

Figure 15: Low Rows...................................................................................................................................... 98

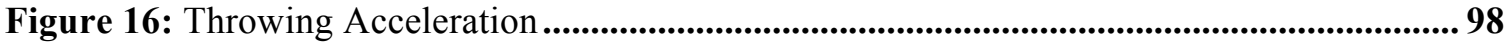

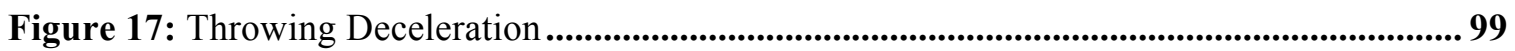

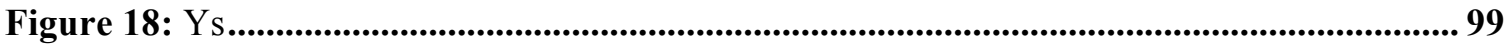

Figure 19: Ts ............................................................................................................................................ 100

Figure 20: Ws ......................................................................................................................................... 100

Figure 21: Scapular Punches............................................................................................................. 100

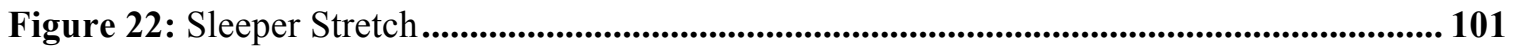

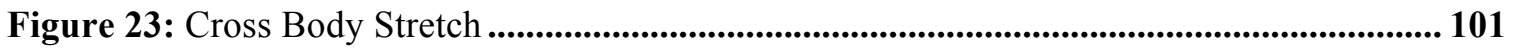

Figure 24: Bony Landmarks of the trunk, scapula, and humerus ................................................. 102 
Figure 25: Scapular position and orientation to be measured ............................................................ 102

Figure 26: Shoulder Flexion strength changes between sessions by group .................................. 103

Figure 27: Shoulder Abduction strength changes between sessions by group ............................ 103

Figure 28: Shoulder Extension strength changes between sessions by group ............................... 104

Figure 29: Shoulder Internal Rotation strength changes between sessions by group .................. 104

Figure 30: Shoulder External Rotation strength changes between sessions by group................. 105

Figure 31: Shoulder Adduction strength changes between sessions by group ............................. 105

Figure 32: Scapular Retraction strength changes between sessions by group ............................. 106

Figure 33: Scapular Retraction with Downward Rotation strength changes

between sessions by group ........................................................................................................................... 106

Figure 34: Scapular Retraction with Upward Rotation strength changes

between sessions by group .......................................................................................................................... 107

Figure 35: Internal-External Rotation changes of session, angle, and group................................. 108

Figure 36: Upward-Downward Rotation changes of session, angle, and group.......................... 109

Figure 37: Anterior-Posterior Tipping changes of session, angle, and group............................... 110

Figure 38: Protraction-Retraction changes of session, angle, and group.................................... 111

Figure 39: Elevation-Depression changes of session, angle, and group ........................................ 112

Figure 40: SANE reporting changes between sessions by group ................................................ 113

Figure 41: DASH reporting changes between sessions by group ................................................. 113 


\section{List of Tables}

Table 1: Reliability of strength assessment ............................................................................114

Table 2: Reliability of scapular kinematic assessment .......................................................................... 114

Table 3: Bony landmarks of the scapula, thorax, and humerus ................................................... 115

Table 4: Definition of local coordinate systems ....................................................................................... 116

Table 5: Summary of exercises and stretches to be performed .................................................... 117

Table 6: Subject Demographics .............................................................................................................. 118

Table 7: Means and Standard Deviations of the Shoulder and Scapular Stabilizer Strength .... 118

Table 8: Means and Standard Deviations of the Scapular Kinematics ........................................... 119

Table 9: Means and Standard Deviations of the Subjective Rankings............................................ 120 


\section{List of Appendices}

Appendix 1: SANE and DASH .............................................................................................................. 121

Appendix 2: Manuscript formatted for the Clinical Journal of Sports Medicine ......................... 122 


\section{CHAPTER I}

\section{INTRODUCTION}

\section{An Overview}

Shoulder pain is a debilitating problem in overhead sports, especially in swimming (Costill, Kovaleski et al. 1985; Johnson 1988; Beach, Whitney et al. 1992; Allegrucci, Whitney et al. 1994; Bak and Fauno 1997; Bak and Magnusson 1997; McMaster, Roberts et al. 1998; McMaster 1999; Troup 1999; Ramsi, Swanik et al. 2004; Layton, Thigpen et al. 2005). Competitive swimmers train approximately 10,000-14,000 meters per day, 6-7 times per week, which correlates to 16,000 shoulder revolutions per week (Stocker, Pink et al. 1995; Pink and Tibone 2000). During the training sessions, significant demand is placed on the shoulder, as $90 \%$ of the propulsive force during swimming comes from the upper extremity, specifically the shoulder (Johnson 1988). Because of the significant number of shoulder revolutions performed during participation, shoulder pain has become commonplace in competitive swimming, comprising at least 55\% of all injuries (McFarland and Wasik 1996). Interfering shoulder pain has been reported in $45-87 \%$ of all swimmers during their careers (Johnson 1988; Beach, Whitney et al. 1992; Stocker, Pink et al. 1995; Urwin, Symmons et al. 1998; Pink and Tibone 2000; Bongers 2001) 


\section{"Swimmer's Shoulder"}

The high frequency and intensity of training often leads to "swimmer's shoulder" which is the general term for a shoulder overuse injury in competitive swimmers (Richardson 1986). “Swimmer's shoulder” has often been cited as an impingement syndrome that leads to shoulder pain and inflammation of the rotator cuff and biceps tendon (Bak and Magnusson 1997). Due to the repetitive nature of overhead swimmers, the repeated contact of the humerus with the rotator cuff and biceps tendons leads to increased risks of impingement. In swimmers, the biomechanics of the freestyle stroke exacerbate impingement symptoms. While completing the freestyle stroke, $25 \%$ of the stroke occurs in a position that causes subacromial impingement (Yanai and Hay 2000). The majority of this impingement occurs during the catch and recovery phases of the stroke. During the catch phase, the swimmer reaches forward, creating a large moment arm and hydrodynamic force that causes the shoulder to elevate beyond the maximum active angle (Yanai, Hay et al. 2000). This forced elevation causes compression in the subacromial space and creates shoulder impingement for approximately $10 \%$ of the stroke time (Yanai and Hay 2000). During the recovery phase, the elbow is held high with abduction and external rotation of the shoulder and the arm is completely relaxed to allow for muscle recovery. The recovery phase is the most common phase for impingement to occur, especially in swimmers who complete the recovery phase with a large internal rotation angle (Yanai, Hay et al. 2000). The repetitive stress placed on the muscles throughout the stroke may lead to shoulder pain and impingement symptoms.

The exact cause of "swimmer's shoulder" is unknown, but possible etiologies include decreased subacromial space width, altered scapular kinematics and associated 
muscle recruitment patterns, posterior shoulder tightness, and altered glenohumeral arthrokinematics (Solem-Bertoft, Thuomas et al. 1993; Deutsch, Altchek et al. 1996; Ludewig, Cook et al. 1996; Wadsworth and Bullock-Saxton 1997; Graichen, Bonel et al. 1999; Lukasiewicz, McClure et al. 1999; Ludewig and Cook 2000; Tyler, Nicholas et al. 2000; Tsai, McClure et al. 2003; Karduna, Kerner et al. 2005; Myers, Laudner et al. 2006; Santos, Belangero et al. 2007).

\section{Physical Profile of Swimmers}

In addition to the repetitive nature of the sport, swimmers have a distinct physical profile that predisposes them to shoulder injury and pain. Swimmers have been found to have an increase of $10^{\circ}$ in external rotation and $40^{\circ}$ in abduction and a decrease of $40^{\circ}$ of internal rotation when compared to non-swimmers (Beach, Whitney et al. 1992). Altered range of motion in swimmers may predispose swimmers to injuries by altering scapular kinematics and causing abnormal stress on the surrounding musculature (Beach, Whitney et al. 1992; Bak and Fauno 1997; Bak and Magnusson 1997). Since decreased internal rotation range of motion has been linked to a pattern of scapular kinematics that results in narrowing of the subacromial space, decreased internal rotation range of motion is implicated in the development of subacromial impingement in overhead athletes (Borich, Bright et al. 2006; Myers, Laudner et al. 2006). Swimmers have also been found to have increased shoulder internal rotation and adduction strength, due to adaptation to the demands of the sport (Beach, Whitney et al. 1992; McMaster 1994; Bak and Fauno 1997; Bak and Magnusson 1997). As a result, imbalances are created in the internal rotation/external rotation ratio and the abduction/adduction ratio which has been found to 
lead to pain (Costill, Kovaleski et al. 1985). Throughout the approximately 10 months competition season, internal and external rotation strength in swimmers increases, although internal rotation strength gains are significantly more than the external rotation strength gains, further increasing the IR:ER ratio (Ramsi, Swanik et al. 2004; Su, Johnson et al. 2004). This further predisposes swimmers to shoulder injuries later in the season (Ramsi, Swanik et al. 2004). Finally, swimmers are notorious for having poor posture (Johnson 1988; Bak and Fauno 1997). Swimmers are characterized as having forward head, rounded shoulders, and increased thoracic kyphosis, which can affect scapular kinematics, muscle strength, and range of motion (Kebaetse, McClure et al. 1999; Wang, McClure et al. 1999; Finley and Lee 2003).

The repetitive nature of the sport, biomechanics of the freestyle stroke, and physical profile of swimmers predisposes these athletes to overuse shoulder injuries, which may require them to take time off to allow for healing. While rest may be beneficial to treat the injury, significant detraining can occur as little as one week of decreased activity (Costill, Fink et al. 1985; Neufer 1989). Because of the detraining that can occur with rest, it is paramount to develop a shoulder injury prevention program for swimmers to address the strength deficits and altered pattern of scapular kinematics that have been found to lead to injury.

Few injury prevention program studies have been conducted specifically for swimmers. Swanik et al (Swanik, Swanik et al. 2002) implemented a 6-week functional training program for swimmers and found significantly less reported incidences of pain in swimmers who completed the training program compared to a control group. Although this study did not look at specific variables that may have contributed to their pain, it 
provides a good framework for a prevention program to be developed. Other studies in swimmers aimed to determine the effect of an intervention program on a specific variable (McMaster 1994; Wang, McClure et al. 1999; Swanik, Lephart et al. 2002). Kluempler et al (Kluemper, Uhl et al. 2006) evaluated the effectiveness of a 6-week stretching and strengthening program on forward shoulder posture. After the 6-week intervention, swimmers in the treatment group had significantly decreased forward shoulder posture than swimmers in the control group. This study found that a training program can be beneficial in improving posture and that further research needs to be conducted to determine the effectiveness of an intervention program on other variables related to pain, such as range of motion, strength, and scapular kinematics. A more general study by McClure et al (McClure, Bialker et al. 2004) evaluated the effect of a 6-week exercise program on individuals with shoulder impingement syndrome and found improvement in passive internal rotation and external rotation range of motion, abduction and internal rotation strength, and pain levels. While this study provides valuable information, participants did not have the physical characteristics or the daily demands of a collegiate swimmer, and these factors may alter the results of the intervention.

\section{Purpose and Clinical Relevance}

While studies have found that swimmers possess characteristics that make them vulnerable to impingement, no study has evaluated a prevention program designed specifically for swimmers that addresses the known weaknesses of these athletes. To date, a research-based prevention program to addresses the strength and altered scapular kinematics of collegiate swimmers has not been developed. The purpose of this study was 
to determine the effects of a 6-week intervention program on shoulder strength and scapular kinematics in division I collegiate swimmers. Validating a shoulderstrengthening and stretching program for collegiate swimmers to decrease the risk of shoulder impingement will provide clinicians, coaches, and athletes with a specific program to incorporate into their training and potentially reduce the incidence of shoulder pain during a competitive season.

\section{Research Questions}

What is the effect of a 6-week shoulder strengthening program on shoulder and scapular stabilizer strength and scapular kinematics in division I collegiate swimmers?

- $R Q 1$ : What is the effectiveness of the six-week strengthening program in improving shoulder strength?

○ $R Q$ 1.1: What is the effectiveness of the six-week intervention program in improving shoulder flexion strength?

O $R Q$ 1.2: What is the effectiveness of the six-week intervention program in improving shoulder extension strength?

$\circ R Q$ 1.3: What is the effectiveness of the six-week intervention program in improving shoulder abduction strength?

○ $R Q$ 1.4: What is the effectiveness of the six-week intervention program in improving shoulder adduction strength?

○ $R Q$ 1.5: What is the effectiveness of the six-week intervention program in improving shoulder internal rotation strength?

○ $R Q$ 1.6: What is the effectiveness of the six-week intervention program in 
improving shoulder external rotation strength?

- $R Q 2$ : What is the effectiveness of the six-week intervention program in improving scapular stabilizer strength?

- RQ 2.1: What is the effectiveness of the six-week intervention program in improving scapular retraction with upward rotation (Y) strength?

- RQ 2.2: What is the effectiveness of the six-week intervention program in improving scapular retraction (T) strength?

- RQ: 2.3: What is the effectiveness of the six-week intervention program in improving scapular retraction with downward rotation (W) strength?

- $R Q 3$ : What is the effectiveness of the six-week intervention program in improving scapular kinematics during an elevation task in the scapular plane?

- $R Q$ 3.1: What is the effectiveness of the six-week intervention program in increasing scapular upward rotation during an elevation task in the scapular plane?

- $R Q$ 3.2: What is the effectiveness of the six-week intervention program in increasing scapular posterior tipping during an elevation task in the scapular plane?

- $R Q$ 3.3: What is the effectiveness of the six-week intervention program in increasing scapular external rotation during an elevation task in the scapular plane?

- $R Q$ 3.4: What is the effectiveness of the six-week intervention program in increasing scapular retraction during an elevation task in the scapular plane? 
○ $R Q$ 3.5: What is the effectiveness of the six-week intervention program in increasing scapular elevation during an elevation task in the scapular plane?

- $R Q 4$ : What is the effectiveness of the six-week intervention program in improving subjects' shoulder functioning and overall satisfaction?

\section{Research Design}

Randomized control trial with an intervention group and control group.

\section{Dependent Variables}

Strength (Peak force measured with a handheld dynamometer during a break test)

- Shoulder Strength
$\circ$ Flexion
○ Extension
- Internal Rotation
○ External Rotation
- Abduction
○ Adduction

- Scapular Stabilizer Strength

- Scapular Retraction

- Scapular Retraction with downward rotation

- Scapular Retraction with upward rotation

- Scapular Kinematics (Measured at $0^{\circ}, 30^{\circ}, 60^{\circ}, 90^{\circ}$, and $120^{\circ}$ of humeral elevation in the scapular plane during the ascending phase)

- Scapular upward/downward rotation 
- Scapular anterior/posterior tipping

- Scapular internal/external rotation

- Scapular protraction/retraction

- Scapular elevation/depression

- Function and satisfaction

- Sports module of the Disabilities of the Arm, Shoulder, and Hand (DASH)

- Single Assessment Numeric Evaluation (SANE) of the shoulder

\section{Independent Variable}

Intervention Program

- Strengthening Exercises completed using resistive tubing

- Scapular Retraction with Downward Rotation (Ys)

- Scapular Retraction (Ts)

- Scapular Retraction with Upward Rotation (Ws)

- Shoulder Flexion

○ Shoulder Extension

○ Low Rows

- Shoulder Internal Rotation at $90^{\circ}$ abduction

- Shoulder External Rotation at $90^{\circ}$ abduction

○ D2 pattern Acceleration

- D2 pattern Deceleration

- Scapular Punches

- Stretching Exercises

- Sleeper Stretch 


\section{○ Corner Stretch}

\section{Hypotheses}

After completing the training protocol for 6 weeks, swimmers in the intervention group will exhibit greater strength of shoulder musculature, scapular stabilizers, and more efficient scapular kinematics than individuals in the control group.

- H1: After the six-week intervention program, there will be an increase in strength of shoulder musculature (Flexion, extension, ER, IR, ABD, ADD) in division I collegiate swimmers who completed the training program compared to the controls.

○ H 1.1: Swimmers who completed the intervention program will exhibit an increase in shoulder flexion strength.

○ $H$ 1.2: Swimmers who completed the intervention program will exhibit an increase in shoulder extension strength.

○ $H$ 1.3: Swimmers who completed the intervention program will exhibit an increase in shoulder abduction strength.

○ $H$ 1.4: Swimmers who completed the intervention program will exhibit an increase in shoulder adduction strength.

○ H 1.5: Swimmers who completed the intervention program will exhibit an increase in shoulder internal rotation strength.

○ H 1.6: Swimmers who completed the intervention program will exhibit an increase in shoulder external rotation strength.

- H2: After the six-week intervention program, there will be an increase in muscular strength of the scapular stabilizers in division I collegiate swimmers 
who completed the training program compared to the controls.

- $H$ 2.1: Swimmers who completed the intervention program will exhibit an increase in scapular retraction (T) strength.

- $H$ 2.2: Swimmers who completed the intervention program will exhibit an increase in scapular retraction with upward rotation (Y) strength.

○ $\quad H$ 2.3: Swimmers who completed the intervention program will exhibit an increase in scapular retraction with downward rotation (W) strength.

- H3: After the six-week intervention program, the division I collegiate swimmers will exhibit an improvement in scapular kinematics.

○ H3.1: Swimmers who completed the intervention program will exhibit an increase in upward rotation (UR) of the scapula.

○ H3.2: Swimmers who completed the intervention program will exhibit an increase in posterior tipping (PT) of the scapula.

○ H3.3: Swimmers who completed the intervention program will exhibit an increase in external rotation (ER) of the scapula.

○ H3.4: Swimmers who completed the intervention program will exhibit an increase in scapular retraction (SR).

- H3.5: Swimmers who completed the intervention program will exhibit an increase in scapular elevation (SE).

- H4: After the six-week intervention program, the swimmers in the intervention program will exhibit an improvement in SANE and DASH scores. 


\section{Null hypothesis:}

- The six week intervention program designed specifically to address the needs of division I collegiate swimmers will not result in greater improvement in shoulder muscles strength, scapular stabilizing muscles strength, or scapular kinematics compared to the controlled group.

\section{Operational Definitions}

- Competitive collegiate swimmers: Swimmers on a NCAA division I swimming team

- Scapular Kinematics: Upward/Downward Rotation, Anterior/Posterior Tipping, Internal/External Rotation of the scapula, scapular protraction/retraction, and scapular elevation/depression @ $0^{\circ}, 30^{\circ}, 0^{\circ}, 90^{\circ}$, and $120^{\circ}$ of humeral elevation during a scaption task.

- Shoulder Strength: Average of three trials of the peak force produced during the break test for shoulder flexion, extension, abduction, internal rotation, and external rotation

- Scapular Stabilizer Strength: Average of three trials of the peak force produced during the break test for scapular retraction, scapular retraction with downward rotation, and scapular retraction with upward rotation.

\section{Assumptions}

- A dynamometer is a reliable and precise measure of shoulder strength.

- Electromagnetic Tracking Device is a reliable and precise measure to assess scapular kinematics

- The subjects used in this study are representative of other collegiate swimmers in 
different programs.

- All subjects are performing the same weight lifting program.

- Subjects will follow directions when completing intervention program.

\section{Delimitations}

- Only swimmers on the UNC varsity swim team will be tested to ensure compliance to the exercise program.

- The testers are blinded from the group assignment to eliminate examiner bias.

\section{Limitations}

- The effort that each subject puts into the training cannot be assessed.

- Activities outside of team training cannot be controlled.

- Subjects will not be blinded.

- The effects of the strengthening program over the entire season will not be measured. The long-term benefits of the program will be hypothesized from the results from a 6-week training program. 


\section{CHAPTER II}

\section{REVIEW OF THE LITERATURE}

\section{Shoulder pain in Swimmers}

Swimming is a popular activity that encompasses individuals of all ages that swim for fitness, recreation, and competition. It is estimated that over 100 million Americans swim on a regular basis with USA swimming having over 250,000 registered swimmers, USA Masters Swimming having over 38,000 and high school and summer league teams including over 5 million individuals (Johnson 1988; Johnson, Gauvin et al. 2003). Shoulder pain is a debilitating problem in all overhead sports, especially in swimming (Johnson 1988; Stocker, Pink et al. 1995; Pink and Tibone 2000; Johnson, Gauvin et al. 2003). Ninety percent of the propulsive force during swimming comes from the upper extremity, specifically the shoulder (Johnson 1988). The demand placed on the shoulder due to the frequency of revolutions while swimming is significantly greater than in any other overhead sports. Competitive swimmers train approximately 10,000-14,000 meters per day, 6-7 times per week, which correlates to 16,000 shoulder revolutions per week (Stocker, Pink et al. 1995; Pink and Tibone 2000). The number of shoulder revolutions compares with 1000 revolutions per week in a professional tennis player or baseball pitcher, 300 shoulder revolutions per week in a college javelin thrower, and 200 revolutions per week in a professional golfer (Johnson 1988). In addition, survey data has revealed shoulder pain reported in approximately $45-87 \%$ of swimmers, compared to $16-21 \%$ in the general population, $57 \%$ in professional pitchers, 
$44 \%$ in collegiate volleyball players, and 29\% in collegiate javelin throwers (Johnson 1988;

Beach, Whitney et al. 1992; Stocker, Pink et al. 1995; Urwin, Symmons et al. 1998; Pink and Tibone 2000; Bongers 2001). At least 55\% of all injuries in swimming are due to shoulder pain, which has become commonplace in competitive swimming and more frequent than in other overhead sports, due to the significant number of shoulder revolutions performed per week (McFarland and Wasik 1996).

\section{Shoulder Injuries}

\section{Subacromial Impingement}

Subacromial impingement is also commonly called external impingement and is a mechanical compression of the rotator cuff tendons, biceps tendon, or subacromial bursa by the acromion, which causes pain, loss of range of motion and decreased strength and functioning (Neer 2005). The coracoacromial arch is made of the anterior acromion, coracoacromial ligament and the acromioclavicular joint. In shoulder impingement syndrome, the subacromial space, the area beneath the coracoacromial arch, becomes

compressed (Neer 1983; Graichen, Bonel et al. 1999). The contents of the subacromial space include: the supraspinatus, long head of the biceps and the subacromial bursa. The other rotator cuff muscles, the infraspinatus, teres minor and subscapularis, are also susceptible to being impinged between the humeral head and the undersurface of the acromion as they become confluent with the glenohumeral capsule (Michener, McClure et al. 2003; Moore and Dalley 2006). Compression of any of these structures may lead to pain and dysfunction, especially in an overhead athlete (Neer 1983; Fu, Harner et al. 1991; Graichen, Bonel et al. 1999; Michener, McClure et al. 2003; Wilk, Obma et al. 2009).

Subacromial impingement can be classified as primary impingement or secondary 
(Neer 1983; Fu, Harner et al. 1991; Neer 2005). Primary external impingement is the irritation of the rotator cuff due to mechanical narrowing of the subacromial space due to subacromial spurring or an altered shape of the acromion (Meyer 1931; Neer 2005). These bony deformities affect the amount of space available in the subacromial space and increase the incidence of impingement (Bigliani, Morrison et al. 1986; MacGillivray, Fealy et al. 1998). There are three types of acromion shape. A type 1 acromion is flat and has a low incidence of impingement, type 2 is curved and has a greater incidence of impingement and type 3 is hooked and has the highest incidence of impingement (Bigliani, Morrison et al. 1986; MacGillivray, Fealy et al. 1998; Worland, Lee et al. 2003). Tendon degeneration occurs as the rotator cuff becomes frayed or irritated (Soslowsky, Thomopoulos et al. 2002; Ko, Huang et al. 2006). Tendinosis and associated tears in individuals with primary subacromial impingement most commonly occur in older athletes at the anterior half of the supraspinatus tendon (Tuite, Turnbull et al. 1998). Radiological examination is necessary to confirm the diagnosis of primary external impingement (Belzer and Durkin 1996). Treatment of primary external impingement is typically surgical before the weaknesses and decreased range of motion of the rotator cuff can be addressed (Belzer and Durkin 1996).

Secondary subacromial impingement is when the subacromial structures become compressed as a result of functional narrowing of the subacromial space (Cohen and Williams 1998). Weak or altered activation patterns of the rotator cuff and scapular stabilizer muscles causes an inability to keep the humeral head centered in the glenoid during movement (Hawkins, Misamore et al. 1985; Hardy, Vogler et al. 1986; Deutsch, Altchek et al. 1996; Graichen, Bonel et al. 1999). During movement, the humeral head narrows the subacromial space leading to increased compression of the structures within the subacromial 
space (Fu, Harner et al. 1991; Deutsch, Altchek et al. 1996; Graichen, Bonel et al. 1999). Secondary subacromial impingement is more likely to occur in younger athletes, who will report anterior shoulder pain as the primary symptom (Tuite, Turnbull et al. 1998). While surgery is usually required for an individual with primary subacromial impingement, the treatment goal for an individual with secondary external impingement is to address the underlying instability, which can often be done with an effective strengthening and stretching program (Arroyo, Hershon et al. 1997).

The repetitive stress on the biceps tendon and the rotator cuff from all types of impingement can lead to rotator cuff and biceps tendinosis (Soslowsky, Thomopoulos et al. 2002; Andrews, Wilk et al. 2008; Krupp, Kevern et al. 2009; Wilk, Obma et al. 2009).

\section{Rotator Cuff Tendinosis}

The rotator cuff is made up of the supraspinatus, infraspinatus, subscapularis and teres minor. The rotator cuff is primarily responsible for stabilizing the shoulder by holding the head of the humerus in the glenoid fossa (Moore and Dalley 2006). Rotator cuff tendinosis is a chronic intratendonous disease that includes chronic inflammation, degeneration, or tearing in the rotator cuff (Wilk, Obma et al. 2009). Degeneration of the rotator cuff tendons occurs most often in overhead sports due to the repetitive impingement and resultant microtrauma from the movements of the shoulder during athletic activity (Chang 2004). Rotator cuff tendinosis has been found to be more common in overhead athletes with improper functioning of the scapula (Voight and Thomson 2000). The structural damage to the rotator cuff typically occurs in the supraspinatus and sometimes the infraspinatus resulting in weakness of these muscles (Donatelli 2004; Wilk, Obma et al. 2009). Individuals with rotator cuff tendinosis will experience a deep, achy pain that 
becomes worse as the arm is elevated (Graichen, Bonel et al. 1999; Opsha, Malik et al. 2008). MRI imaging will show intrasubstance wear on the tendon, indicating chronic irritation (Opsha, Malik et al. 2008; Wilk, Obma et al. 2009). Rotator cuff tendinosis can be treated by rest followed by strengthening of the rotator cuff muscles to restore the muscles' function in stabilizing humeral head (Donatelli 2004). While minor rotator cuff tendinosis can be easily treated, chronic tendonitis can lead to a tear in the rotator cuff muscles that may need to be treated surgically (Chang 2004).

\section{Biceps Tendinosis}

The biceps brachii is a large muscle located on the anterior surface of the humerus. The biceps helps to stabilize the head of the humerus in the glenoid fossa through compression and depression, as well as aid in acceleration and deceleration of the arm during overhead activities (Kumar, Satku et al. 1989; Rodosky, Harner et al. 1994; Pagnani, Deng et al. 1996; Andrews, Wilk et al. 2008; Krupp, Kevern et al. 2009; Wilk, Obma et al. 2009). The long head of the biceps is particularly vulnerable to injury from impingement due to its location in the subacromial space (Moore and Dalley 2006). Biceps tendinosis is an overuse injury that occurs in overhead athletes due to the repetitive nature of the sport and can be classified as inflammatory/degenerative condition (Krupp, Kevern et al. 2009). Individuals with biceps tendinosis will have localized tenderness and crepitus of the biceps tendon and will have pain with overhead activities. Conservative rehabilitation of rest, NSAIDs and strengthening is typically successful (McMaster 1999). Surgical intervention is usually only required for chronic cases or in older individuals (McMaster 1999).

\section{"Swimmer's Shoulder"}

"Swimmer's shoulder" is the general term for a shoulder overuse injury, which 
encompasses subacromial impingement, rotator tendinosis, and biceps tendinosis (Richardson 1986; Bak and Magnusson 1997). The development of "swimmer's shoulder" is complex and can be attributed to a variety of factors, including: decreased subacromial space width, altered scapular kinematics, altered muscle recruitment pattern, posterior shoulder tightness, and humeral head displacement (Solem-Bertoft, Thuomas et al. 1993; Deutsch, Altchek et al. 1996; Ludewig, Cook et al. 1996; Wadsworth and Bullock-Saxton 1997; Graichen, Bonel et al. 1999; Lukasiewicz, McClure et al. 1999; Ludewig and Cook 2000; Tyler, Nicholas et al. 2000; Tsai, McClure et al. 2003; Karduna, Kerner et al. 2005; Myers, Laudner et al. 2006; Santos, Belangero et al. 2007). In addition to these causes of shoulder injury, swimmers have certain physical characteristics that further predispose them to impingement syndrome (Costill, Kovaleski et al. 1985; Hawley and Williams 1991; Beach, Whitney et al. 1992; Zemek and Magee 1996; Bak and Fauno 1997; Bak and Magnusson 1997; McMaster, Roberts et al. 1998; McMaster 1999; Troup 1999; Johnson, Gauvin et al. 2003; Ramsi, Swanik et al. 2004; Layton, Thigpen et al. 2005). Swimmers must have increased shoulder range of motion, increased internal rotation and adduction strength, participate in prolonged, fatiguing training and generate repetitive, forceful muscle contractions in order to be successful (Stocker, Pink et al. 1995; Weldon and Richardson 2001). While these distinct factors may improve the level of success of a swimmer, they can also lead to inflammation and pain (Beach, Whitney et al. 1992).

\section{Etiology of "Swimmer's Shoulder"}

\section{Decreased Subacromial Space Width}

The subacromial space is an area beneath the acromion, with the head of the humerus serving as the floor and the coracoacromial ligament as the roof (Fu, Harner et al. 1991). The 
subacromial bursa, tendons of the rotator cuff and biceps tendon are contained in the subacromial space. As shoulder movement occurs, the size of the subacromial space changes. Solem-Bertoft et al (Solem-Bertoft, Thuomas et al. 1993) found that the subacromial space narrowed as the shoulder moved from a retracted position to a protracted position. Narrowing of the subacromial space results in increased contact between the humerus and the underlying structures (Fu, Harner et al. 1991; Solem-Bertoft, Thuomas et al. 1993). In a study investigating the width of the subacromial space through Magnetic Resonance imaging (MRI), it was found that patients with early stages of impingement syndrome showed a significant decrease in the acromiohumeral and claviculohumeral distance during muscle activity (Graichen, Bonel et al. 1999). This study functionally evaluates the subacromial space, finding a functional deficit of the supraspinatus in patients with impingement syndrome that occurs because of decreased subacromial space when the shoulder musculature comes in contact with the humerus. As the width of the subacromial space is decreased, compression of the contents occur, which has a significant impact on the development of shoulder impingement syndrome and is most likely related to incidences of shoulder pain and decrease in range of motion and muscle strength (Fu, Harner et al. 1991).

\section{Altered Scapular Kinematics}

The scapula is the cornerstone of upper extremity movement and its primary role is to ensure proper position and motion for optimal shoulder function (Kibler and McMullen 2003). Movement of the scapula relative to the thorax is described as scapular kinematics.

The 5 movements included in scapular kinematics are: scapular upward/downward rotation, scapular internal/external rotation, scapular anterior/posterior tipping, clavicular protraction/retraction, and clavicular elevation/depression (Karduna, McClure et al. 2000; 
Karduna, McClure et al. 2001; Borich, Bright et al. 2006; Ludewig and Reynolds 2009). In a healthy individual, upward rotation, posterior tipping and decreased internal rotation, retraction, and elevation is the common movement pattern as the humeral angle increases (Ludewig, Cook et al. 1996; Karduna, McClure et al. 2001; Ludewig and Reynolds 2009). Upward rotation of the scapula serves to elevate the lateral acromion in order to prevent impingement and is the primary scapular motion (Ludewig and Reynolds 2009). Posterior tipping of the scapula is a secondary scapular motion and moves the anterior acromion posteriorly in order to prevent impingement of the rotator cuff tendons (Ludewig and Reynolds 2009). External rotation is an accessory motion of the scapula, which moves the acromion posteriorly to decrease contact with the rotator cuff tendons (Ludewig and Reynolds 2009). Clavicular kinematics defines the position of the scapula relative to the thorax and is used to determine the amount of scapular elevation/depression and protraction/retraction. The understanding of the role of normal clavicular kinematics and their effect on the development of shoulder pain are limited. It has been found that individuals have slight clavicular elevation and retraction at rest. During flexion, increased clavicular elevation occurred, while there was no change in clavicular retraction. During abduction in the coronal and scapular planes, there was an increase in both clavicular elevation and retraction. These studies showed the clavicular elevation and retraction are an integral part of shoulder movement during passive humeral elevation (Ludewig, Behrens et al. 2004).

Three-dimensional scapular kinematics is commonly measured using an electromagnetic tracking device (Karduna, McClure et al. 2001). Karduna et al (Karduna, McClure et al. 2001) conducted a validation study where an electromagnetic tracking sensor 
was placed directly over the acromion and compared with the invasive measure of scapular kinematics. This study found that this non-invasive way of measuring scapular kinematics is valid when compared to an invasive method, when the arm is below $120^{\circ}$ of elevation. This measurement can measure scapular orientation in regards to upward/downward rotation, internal/external rotation, and anterior/posterior tipping. Clavicular movement can be measured indirectly, which represents the scapular position regarding protraction/retraction and elevation/depression. Clavicular movement has not been studied as well as scapular kinematics, but may be important for overall shoulder movement. Indirect measurement of clavicular kinematics using electromagnetic tracking has also been found to be reliable and valid (Karduna, McClure et al. 2001; Ludewig, Behrens et al. 2004).

Normal movements of the scapula have been found to be altered in individuals who present with shoulder pain. Several studies have shown alterations in scapular kinematics with the presence of impingement syndrome (Lukasiewicz, McClure et al. 1999; Ludewig and Cook 2000; Endo, Ikata et al. 2001; Ludewig and Reynolds 2009). In a study by Lukasiewicz et al (Lukasiewicz, McClure et al. 1999), significantly less posterior tilt was found in symptomatic patients when compared to the uninvolved side and asymptomatic subjects. This study found no significant difference in the amount of scapular upward rotation or internal rotation, although only static positions were measured. Therefore assessment of scapular kinematics during functional measurements may have different results. In a study by Ludewig and Cook (Ludewig and Cook 2000), subjects with impingement syndrome were found to have decreased upward rotation, decreased posterior tilt and an increase in internal rotation between symptomatic and asymptomatic individuals. The decreased scapular upward rotation and posterior tilt and increased internal rotation 
found in this study, are consistent with what other studies have observed (Hebert, Moffet et al. 2002; Lin, Hanten et al. 2005; McClure, Michener et al. 2006). These findings have also been confirmed by radiological and MRI studies (Graichen, Bonel et al. 1999; Endo, Ikata et al. 2001).

Overhead athletes and specifically swimmers may have increased susceptibility to altered scapular kinematics due to fatigue (Tsai, McClure et al. 2003; Su, Johnson et al. 2004). After implementing a fatigue protocol, increased scapular anterior tilt, internal rotation and downward rotation were observed (Tsai, McClure et al. 2003). Su et al (Su, Johnson et al. 2004) found that swimmers with impingement experienced less upward rotation after practice. This pattern of altered scapular kinematics may cause individuals to be more susceptible to injury later in practice because of the increased contact of the acromion and decreased subacromial space (Ludewig and Cook 2000; Michener, McClure et al. 2003; Ludewig and Reynolds 2009). In a study to determine the effect of scapular orientation on subacromial contact forces, Karduna et al (Karduna, Kerner et al. 2005) found a significant decrease in subacromial clearance resulting from increased scapular upward rotation. The authors suggest that the decreased scapular upward rotation seen in subjects with impingement my be a compensatory adaptation in order to open up the subacromial space and create less compression of the contents of the subacromial space.

Altered scapular kinematic patterns are due to biomechanical and neuromuscular abnormalities that affect the movements of the scapula (Ludewig and Cook 2000; Kibler and McMullen 2003). Diminished serratus anterior activation has been associated with decreased scapular upward rotation (Ludewig and Cook 2000; Lin, Hanten et al. 2005). Decreased serratus anterior activity has also been found to be related to the development of shoulder 
pain (Peat and Grahame 1977; Scovazzo, Browne et al. 1991; Cools, Declercq et al. 2007).

Thus, decreased serratus anterior activity is linked to both decreased upward rotation and shoulder pain. This information provides clinicians with valuable information about developing rehabilitation program. Improving serratus anterior strength may improve scapular kinematics and decrease the likelihood of shoulder pain (Allegrucci, Whitney et al. 1994). Increased clavicular elevation has been found to occur due to excessive upper trapezius activity (Ludewig and Reynolds 2009). Decreased scapular upward rotation and posterior tilt has been found with an overactive upper trapezius, which increases the likelihood for irritation of the rotator cuff and biceps tendons and development of impingement (Ludewig and Cook 2000). Pectoralis minor tightness has been associated with increased scapular internal rotation and anterior tilt (Burkhart, Morgan et al. 2003). This may be due to the fact that individuals with a tight pectoralis major or minor tend to have forward shoulder posture, which places the scapula in an altered starting position. As humeral elevation occurs, the scapula behaves in a unique manner to be able to allow the individual to obtain maximal range of motion. In addition, the tight pectoralis minor provides resistance to the normal upward rotation, posterior tilting, and external rotation that is inherent during humeral elevation (Burkhart, Morgan et al. 2003). Finally, a tight posterior shoulder has also been found to alter scapular kinematics (Borich, Bright et al. 2006). Individuals with a tight posterior shoulder have been found to have increased anterior tilting during elevation, as the tight posterior shoulder structures pulls the scapula anteriorly (Kibler 1998; Borich, Bright et al. 2006). As discussed above, studies have identified specific variables that may result in altered kinematics, which can provide valuable information to create an effective intervention program that addresses muscle imbalances and tightness to 
indirectly improves scapular kinematics, leading to a decrease in pain.

\section{Altered Muscle Recruitment Patterns}

In a study by Wadsworth and Bullock-Saxton (Wadsworth and Bullock-Saxton 1997), the recruitment patterns of the scapular rotator muscles were evaluated in freestyle swimmers with subacromial impingement and control subjects. Participants performed a bilateral elevation of the arms in the plane of the scapula while velocity, posture and degree of postural sway was controlled. The study found that a significantly increased time for activation occurred in swimmers with subacromial impingement, with the greatest delay occurring in the serratus anterior. The delayed recruitment of the serratus anterior would compromise stabilization of the scapula during abduction as well as decreasing the subacromial space (Wadsworth and Bullock-Saxton 1997). Cools et al (Cools, Declercq et al. 2007) investigated the effects of impingement on muscle recruitment of the trapezius. This study found that there was a delay in muscle activation of the middle and lower trapezius muscles in individuals with impingement.

Many studies have aimed to find the effect of shoulder instability on muscle activation patterns. Illyés and Kiss (Illyes and Kiss 2006) found that subjects with multidirectional instability had decreased time of activation of all three portions of the deltoid muscle and pectoralis major during an elevation task compared to controls. In order to compensate for the shorter activation of these muscles, the supraspinatus, infraspinatus, biceps brachii, and triceps brachii have longer activation times. The authors of this study suggest that the shorter activation times of the deltoid and pectoralis major and longer activation times of the other muscles in individuals with multidirectional instability occurs in order to centralize the head of the humerus in the glenoid fossa. While the head of the 
humerus may be centered, these altered patterns of activation significantly affect the scapular kinematics in individuals with multidirectional instability and yield a greater humeral head displacement in these individuals compared with controls during an elevation task (Illyes and Kiss 2006). In a similar experiment by Santos (Santos, Belangero et al. 2007), no significant difference in the time of recruitment or recruitment order was found between individuals with and with out shoulder instability. The authors of this study report that the findings indicate that joint instability alone may not cause alterations in muscle activity, scapular kinematics, time, and recruitment order.

In a unique study by Falla et al (Falla, Farina et al. 2007) the effect of pain on the activation of the trapezius muscle was investigated. Researchers injected subjects with a sterile hypertonic saline to create experimental pain. This solution mimicked pain that may occur during typical shoulder injuries. This study found a decrease in activity of the painful portion of the trapezius, as well as an increase in activity of the pain-free areas. This study shows that individuals who are injured may have decreased muscle activity simply due to pain (Farina, Arendt-Nielsen et al. 2004). Delayed muscle activation of the trapezius and serratus anterior may be related to neuromuscular adaptations that occur as a result of pain.

\section{Posterior Shoulder Tightness}

Posterior shoulder tightness is another factor that contribute to shoulder impingement and is caused by a tight posterior capsule, posterior cuff, and deltoids that limits the amount of glenohumeral internal rotation and horizontal adduction (Harryman, Sidles et al. 1990; Warner and Beim 1997; Tyler, Nicholas et al. 2000; Grossman, Tibone et al. 2005; Borich, Bright et al. 2006; Myers, Laudner et al. 2006). Posterior shoulder tightness may cause alterations in normal glenohumeral arthokinematics that can lead to injury (Harryman, Sidles 
et al. 1990; Warner, Allen et al. 1997; Grossman, Tibone et al. 2005). Posterior shoulder tightness has been linked to subacromial impingement (Warner, Micheli et al. 1990), posterior impingement (Tyler, Nicholas et al. 2000; Myers, Laudner et al. 2006), SLAP lesion (Burkhart, Morgan et al. 2003) and even ulnar collateral ligament injury in baseball players (Dines, Frank et al. 2009). Harryman et al (Harryman, Sidles et al. 1990) found that individuals with posterior shoulder tightness possessed increased and earlier anterior translation of the humeral head during passive flexion and internal rotation. Individuals with impingement in both the dominant and non-dominant arm had increased posterior shoulder tightness and decreased internal range of motion compared with control (Tyler, Nicholas et al. 2000).

One study that evaluated the posterior shoulder tightness specifically in throwing athletes, found that individuals with internal impingement had significantly greater glenohumeral internal rotation deficit and posterior shoulder tightness compared with control subjects when measured with cross-body horizontal adduction ROM (Myers, Laudner et al. 2006). Another study done to determine the effects of posterior shoulder tightness on the throwing shoulder used cadavers to show that individuals with tight posterior shoulder capsules and decreased internal rotation range of motion had abnormal movement of the humerus during external rotation (Grossman, Tibone et al. 2005). The humerus in the cadavers with posterior shoulder tightness moved in a posterosuperior direction instead of the typical posteroinferior direction, increasing the chances for impingement of the rotator cuff tendons and labral tears (Grossman, Tibone et al. 2005). In addition to range of motion deficits and abnormal glenohumeral arthrokinematics, a tight posterior shoulder structures can also adversely affect scapular kinematics. A study by Borich et al (Borich, Bright et al. 
2006) found that overhead athletes with decreased internal rotation range of motion had significantly greater anterior tilt with shoulder internal and external rotation when the arm was at $90^{\circ}$ of shoulder flexion and at $90^{\circ}$ of shoulder abduction. Increased anterior tilt has been found to be a risk factor for the development of shoulder pain due to additional stress placed on the rotator cuff tendons and the labrum (Lukasiewicz, McClure et al. 1999; Ludewig and Cook 2000; Tsai, McClure et al. 2003; Ludewig and Reynolds 2009). In overhead athletes with a tight posterior shoulder, the nature of the throwing motion further exacerbates these symptoms and plays a significant role in development of shoulder pain.

While the posterior shoulder tightness is commonly assessed by measuring glenohumeral internal rotation range of motion, recent studies have demonstrated that the measurement of internal rotation range of motion is largely affected by the osseous adaptation, or humeral torsion (Crockett, Gross et al. 2002; Reagan, Meister et al. 2002; Sabick, Torry et al. 2004; Sabick, Kim et al. 2005; Myers, Oyama et al. (In Press)). Humeral torsion or retroversion describes the amount of twisting that occurs along the longitudinal axis of the humerus that result in increased external rotation and decreased internal rotation range of motion (Hernigou, Duparc et al. 2002). Studies have found that throwing athletes, such as baseball and softball, have increased humeral torsion on the dominant side, which has been identified as an adaptation to the stress of excessive external rotation during the throwing motion (Pieper 1998; Crockett, Gross et al. 2002; Osbahr, Cannon et al. 2002; Sabick, Kim et al. 2005). The humeral torsion allows for increased external rotation during the throwing motion before the anterior capsule and ligaments provide restraint from further motion (Sabick, Kim et al. 2005). Traditional measurements of rotation range of motion do not account for bony adaptations and therefore may not reflect the soft tissue tightness. 
Crockett et al (Crockett, Gross et al. 2002) found that experienced professional baseball pitchers had significantly greater humeral torsion in the dominant shoulder than the nondominant shoulder and when compared to a non-throwing control group. In addition, this study found that in the dominant arm, pitchers had significantly less internal rotation and significantly greater external rotation that in the non-dominant arm (Crockett, Gross et al. 2002). A study by Reagan et al (Reagan, Meister et al. 2002) found the same results in collegiate baseball pitchers as the Crockett et al study found in professional baseball players. Together these studies suggests that the humeral torsion plays a significant role in the decrease in internal rotation range of motion and bony changes may limit range of motion more than soft tissue tightness. No research has been conducted on humeral torsion in swimmers, but is important to consider as a possible explanation for substantial internal rotation deficits.

\section{Humeral head displacement}

In a healthy shoulder, the musculature holds the humeral head in the center of glenoid (Bassett, Browne et al. 1990). Superior displacement may occur when muscular imbalances occur, especially in the deltoid muscle (Lewis, Green et al. 2005). During initiation of shoulder abduction, the deltoid muscle's force vector points superiorly and thus does not act a primary mover; however, this force cause superior translation of the humeral head (Lewis, Green et al. 2005). The role of the infraspinatus and supraspinatus during abduction is to produce joint compression to stabilize the shoulder in the glenoid fossa and counteract the superior translation force on the humeral head from the deltoid muscle (Bechtol 1980;

Deutsch, Altchek et al. 1996; Inman, Saunders et al. 1996). A study by Deutsch et al (Deutsch, Altchek et al. 1996) found that patients diagnosed with impingement or general 
shoulder pain had a statistically significant increase in superior displacement of the humeral head compared to control subjects. This study demonstrates direct link between the superior displacement of the humeral head and the impingement syndrome (Deutsch, Altchek et al. 1996).

\section{Physical Profile of Swimmers}

\section{Range of Motion in Swimmers}

A direct positive relationship has been found between the amount of shoulder laxity and the level of success in swimmers (Zemek and Magee 1996). The increase in range of motion may be advantageous to the swimmer, but reduces stability in the glenohumeral joint due to capsuloligamentous laxity, which can create excessive translation and affect the length-tension relationships of the muscles that provide joint stability and thus maximum force production of the rotator cuff muscles (McMaster, Roberts et al. 1998). McMaster et al (McMaster, Roberts et al. 1998) found a statistically significant relationship between increased shoulder laxity and shoulder pain in elite swimmers. A certain amount of shoulder laxity is necessary for maximum mechanical efficiency during swimming thus, successful swimmers may be a preselected group that is vulnerable to developing further laxity from activity and resultant disability (McMaster 1999). These changes in the range of motion of swimmers is most likely explained as a physiological adaptation to the repetitive stress imposed by the demands of training and predisposes swimmers to chronic shoulder pain and injury (McMaster 1999).

Swimmers have been found to have altered forward elevation and internal and external rotation ranges of motion compared to non-swimmers (McMaster, Roberts et al. 1998; Troup 1999; Layton, Thigpen et al. 2005). Increased forward elevation range of motion 
is a characteristic of all elite swimmers and provides the athlete with a mechanical advantage while swimming (Beach, Whitney et al. 1992; McMaster, Roberts et al. 1998; Troup 1999). When more forward elevation is achieved, a body angle of $180^{\circ}$ can be obtained, making the body parallel to the surface of the water, decreasing drag and allowing the body to move through the water with decreased resistance (McMaster, Roberts et al. 1998; Troup 1999). Swimmers have also been found to have a $10^{\circ}$ increase in external rotation and $40^{\circ}$ increase in abduction compared with non-swimmers, as well as having a $40^{\circ}$ decrease in internal rotation compared to non-swimmers (Beach, Whitney et al. 1992). In overhead athletes, decreased internal rotation range of motion indicates a tight posterior shoulder or osseous adaptations that are associated with injuries (Borich, Bright et al. 2006; Myers, Laudner et al. 2006). In a study by Lin et al (Lin, Lim et al. 2006) posterior shoulder tightness was found to be associated with increased superior translation and decreased posterior translations of the humeral head, which would cause the humeral head to be closer to the rotator cuff tendons and potentially lead to impingement during activity. Although altered range of motion may put individuals at risk for injury, not all individuals who are hypomobile or hypermobile are painful. In a survey study on the role of shoulder flexibility to shoulder pain, a low and nonsignificant correlation was found between shoulder flexibility and pain (Beach, Whitney et al. 1992). While range of motion plays a role in shoulder pain, it is important to understand other factors that may be related to altered range of motion and how these affect shoulder functioning.

\section{Muscle Strength}

The upper extremity, primarily the shoulder, is responsible for forward propulsion during swimming. Significant shoulder strength is necessary to propel the body through the 
water. A direct relationship has been found between shoulder strength and swimming speed (Hawley and Williams 1991). Shoulder adduction and elbow extension is the primary movement required to produce the force necessary to move the body during swimming. These movements are produced mostly by the pectoralis major, latissimus dorsi, and triceps (Bak and Magnusson 1997). Because of the contribution from the pectoralis major and latissimus dorsi muscles, swimmers tend to have increased internal rotation and adduction strength (Beach, Whitney et al. 1992; Bak and Fauno 1997; McMaster 1999). In control subjects the conventional concentric external rotation (ER) to concentric internal rotation (IR) strength ratio is .75 , while swimmers have a significantly lower ratio at .64 due to the increased internal rotation strength (Beach, Whitney et al. 1992; Bak and Magnusson 1997). The conventional ratios have not been linked to increased risks of injury, and their role in development of shoulder pain is still unknown. The conventional ER:IR strength ratios compare concentric external rotation strength to concentric internal rotation strength and do not account for the relationship between the agonist and antagonist muscles during movement.

A functional ER: IR strength ratio has been used to compare eccentric external rotational strength and concentric internal rotational strength in order to compare the strength differences during ballistic overhead activity and has been found to be predictive of injury in swimmers (Bak and Magnusson 1997). The functional ER:IR strength ratio may be a more appropriate measure of muscular imbalance, as it measures the external rotators eccentric ability to decelerate the humerus to center the humeral head while the internal rotators are forcefully contracting concentrically, a movement that is common in swimming and all overhead sports. A significant increase in the functional ER:IR ratio on the symptomatic side 
compared to the asymptomatic side, as well as between painful and non-painful groups has been observed in Danish National Swimmers (Bak and Magnusson 1997). This finding indicates that concentric internal rotation strength may be decreased in swimmers who are experiencing shoulder pain. Therefore, rehabilitation and prevention programs need to incorporate exercises that focus on internal rotation strengthening as well as external rotation strengthening, in order to address the needs of the athlete.

Competitive swimmers have organized practice for 10-12 months out of the year with 7-12 practices a week, which can greatly affect the athletes' muscular strength throughout the course of a season (Beach, Whitney et al. 1992). Ramsi et al (Ramsi, Swanik et al. 2004) studied the shoulder rotator strength of high school swimmers over the course of a competitive season. Twenty-seven high school varsity swimmers internal and external strength was measured preseason, midseason, and postseason to detect changes. Internal and external rotation strength significantly increased throughout the season, although internal rotation strength gains were significantly more than the external rotation strength gains, decreasing the functional ER:IR ratio, which has been found to lead to pain in swimmers, and indicates that swimmers may be further predisposed to injury later in the season as the strength gains occur disproportionately (Bak and Magnusson 1997; Ramsi, Swanik et al. 2004).

In addition to having an altered ER:IR ratio, swimmers have been found to have adduction:abduction ratios of 2.05, while the controls ratio was observed as 1.53 (McMaster 1994; Pink and Tibone 2000). In addition, 50 repetitions of isokinetic testing at $240^{\circ} / \mathrm{sec}$ were completed to calculate endurance ratios. The average peak torque of the last 3 trials were divided by the average peak torque of the first 3 trials to determine the strength 
decrement as the muscle fatigued. The adductors endurance ratios were greater than $100 \%$, indicating that the adductors get stronger during activity, while the abductors endurance ratios were approximately $60 \%$ indicating that they were getting weaker as they fatigued. During prolonged activity, the adduction:abduction strength ratio increases and may further predispose an individual to injury (Beach, Whitney et al. 1992).

Swimmers are known to have ER:IR muscle imbalances, as well as adduction:abduction imbalances (Beach, Whitney et al. 1992; McMaster 1994; Bak and Magnusson 1997; Ramsi, Swanik et al. 2004). Furthermore, a correlation between shoulder pain and imbalance of the shoulder musculature has been found in swimmers (Costill, Kovaleski et al. 1985). Muscular imbalances alter the stability of the shoulder, by changing the vectors of pull. The altered vector of pulls result in shoulder instability because of decreased compressive forces on the humerus, which helps to center the humeral head (von Eisenhart-Rothe, Matsen et al. 2005). Shoulder instability can lead to pain, impingement and decreased functioning in overhead athletes (Bassett, Browne et al. 1990; von EisenhartRothe, Matsen et al. 2005; Santos, Belangero et al. 2007).

\section{Muscular Endurance}

Swimmers must possess muscular endurance during each practice session. Significant number of shoulder revolutions are completed each practice and require muscular strength and endurance to maintain shoulder functioning (Pink, Perry et al. 1991; Stocker, Pink et al. 1995). In both healthy swimmers and swimmers with impingement, a $14 \%$ decrease in shrug strength and $13 \%$ decrease in punch strength were detected from pre practice to post practice (Su, Johnson et al. 2004). Swimmers with impingement also showed a decrease in the amount of scapular upward rotation, with greater deficits with increased humeral elevation, 
as they fatigue ( $\mathrm{Su}$, Johnson et al. 2004). These results show that alteration in scapular kinematics and muscle functioning occurred with practice, and swimmers may have increased symptoms later in practice sessions.

\section{Posture}

Anecdotally, swimmers are notorious for having poor posture (Johnson 1988; Bak and Magnusson 1997). Swimmers are characterized as having forward head, rounded shoulders and increased thoracic kyphosis, which can affect scapular kinematics, muscle strength and range of motion (Kebaetse, McClure et al. 1999; Wang, McClure et al. 1999; Finley and Lee 2003). In a study by Kebaetse et al (Kebaetse, McClure et al. 1999) individuals in a slouched posture had significantly less upward rotation and posterior tipping, as well as increased internal rotation of the scapula between $90^{\circ}$ and maximum shoulder abduction occurred when compared to an upright posture. This study also found a $16.2 \%$ decrease in the glenohumeral muscle strength and ability to generate muscle force in the slouched posture.

Although many studies have indicated poor posture as a cause of injury, neither symptomatic nor asymptomatic subjects with impingement syndrome had set postural deviations (Lewis, Green et al. 2005). Unique alterations were present in each of the individuals. The authors of this study suggest that postural deviations might be from stresses placed on the shoulder during development or osseous asymmetries, instead of postural deviations. The research on the role of posture in "swimmer's shoulder" has been inconclusive and further research needs to be conducted to better understand this factor

(Greenfield, Catlin et al. 1995; Wang, McClure et al. 1999; Finley and Lee 2003; Lewis, Green et al. 2005; Kluemper, Uhl et al. 2006). 


\section{Biomechanics of Swimming}

\section{Stroke in Swimming}

During training, $80 \%$ of practice is done using the freestyle stroke regardless of the primary stroke of the swimmer (Beach, Whitney et al. 1992). Because of the tendency to train with freestyle, this will be the emphasis of the current literature review. The freestyle stroke can be broken into five phases: hand entry, catch, insweep, finish and recovery (Richardson 1986; Yanai, Hay et al. 2000). The entry is the phase where the fingertips enter the water. Proper alignment during the entry is to have the hand between the midline of the body and the shoulder, in front of the head with the thumb pointed downward with the elbow downward. This position allows for decreased drag and proper alignment to begin the catch phase.

After hand entry, the swimmer will reach forward with maximal elevation to catch the water. The proper technique is to flex the wrist $40^{\circ}$ and rotate the palm outward as they push down and out through the water (Richardson 1986). The hand entry phase finishes with the hand moving just beyond the width of the shoulder. This will be the widest hand position during the arm stroke. During this catch phase, the arm position creates a large moment arm and the hydrodynamic force causes the shoulder to elevate beyond the maximum active angle (Yanai, Hay et al. 2000). This forced elevation causes compression in the subacromial space and creates shoulder impingement for approximately $10 \%$ of the stroke time (Yanai and Hay 2000).

The pull phase is the next phase of swimming and can be broken into the insweep and finish phase. The insweep is the narrowest point of the pulling pattern, ending near the 
swimmer's chest. During this phase, the palm of the hand will rotate inward and the hand sweeps inward toward the swimmer, causing internal rotation and adduction of the shoulder. The finish phase is a backward and outward movement of the hand as it clears the body for hand exit. During the entire pull phase, the elbow should always be kept high, by flexing the arm, slightly abducting and internally rotating the shoulder (Yanai, Hay et al. 2000). The high elbow position places the muscles of the shoulder at mechanical advantage, allowing for the maximal force production (Troup 1999). Although this advantage is helpful in swimmers, it narrows the subacromial space leading to an impinged position throughout the pull phase (Yanai, Hay et al. 2000).

Finally, the recovery phase is the time from hand exit to the reentry of the hand into the water. While the elbow is held high in the air with abduction and external rotation of the shoulder, the arm should be relaxed to allow the musculature recovery time. This phase is the most common phase for impingement to occur, affecting those who complete the recovery phase with a large internal rotation angle the most (Yanai and Hay 2000).

During the freestyle stroke, impingement of the rotator cuff tendons occurs, regardless of whether the athlete is experiencing symptoms. Approximately $25 \%$ of the stroke time is spent in an impinged position, as the shoulder is in the painful arc between $10^{\circ}$ and $40^{\circ}$ of elevation (Yanai and Hay 2000). This impinged position most often happens during the pull phase and the recovery phase. During the pull phase, the high elbow position gives the swimmer a mechanical advantage, but also increases the internal rotation angle and thus the amount of time spent in an impingement position (McMaster 1999; Yanai, Hay et al. 2000). Furthermore, this explains why many individuals with shoulder pain will begin to swim with a dropped elbow. Dropping the elbow decreases the internal rotation angle and 
time spent in impingement and may become more comfortable for athletes who are experiencing symptoms (McMaster 1999). In the recovery phase, shoulder abduction and external rotation must occur to get the arm back in position for hand entry. Early initiation of external rotation during the recovery phase decreases the time the swimmer spends in the impingement zone and is important in preventing injury (Yanai and Hay 2000). Furthermore, it has been observed that individuals with decreased internal rotation range of motion will spend more time in an impingement position, thus stretching of the internal rotators will be beneficial for the prevention of developing shoulder pain (Yanai and Hay 2000).

\section{Muscle Activity in the Healthy Shoulder}

Although muscle activity during activity is paramount to understanding injury and rehabilitation programs, few studies have observed the muscle activity in swimming, due to equipment considerations and difficulties in simulating training. In a unique study conducted by Pink et al (Pink, Perry et al. 1991), fine wire EMG and cinematography was used to identify the muscle activity in 12 muscles that occurs during the normal freestyle stroke. This information is very important in understanding the functioning and treatment of the shoulder for swimming athletes.

During the hand entry phase, the upper trapezius, rhomboids, supraspinatus, anterior and middle deltoid and serratus anterior all show peak activity (Pink, Perry et al. 1991). The upper trapezius functions to elevate the scapula, the rhomboids retract the scapula while the serratus anterior is responsible for upward rotation of the scapula. The arm is abducted and flexed by the supraspinatus and anterior and middle deltoid.

During the pulling phase, the pectoralis major, teres minor, latissimus dorsi, subscapularis and serratus anterior reach peak activity (Pink, Perry et al. 1991). The 
pectoralis major is responsible for adducting, extending and internally rotating the arm as the teres minor works as an antagonist to externally rotate the arm. The latissimus dorsi is the second major muscle to fire, causing extension and internal rotation with the assistance of the subscapularis. The deltoids fired successively during the late pull phase, beginning with the posterior deltoid. The deltoids, latissimus dorsi and pectoralis major function to create the force to propel the body through the water (Bak and Magnusson 1997). Throughout the pull phase, the serratus anterior is active and is responsible for maintaining the joint congruency of the scapula with the humerus by upwardly rotating the humerus (Pink, Perry et al. 1991). Because the serratus anterior is active through out the entire stroke, it is prone to fatigue during swimming.

During the transition from pulling to hand exit, the posterior deltoid is the most active, leading to hand exit by the upper trapezius, rhomboids, supraspinatus, anterior and middle deltoid and serratus anterior (Pink, Perry et al. 1991). The middle deltoid and supraspinatus abduct the arm as the anterior deltoid initiate shoulder flexion. The upper trapezius elevates the scapula, the rhomboids upwardly rotate the scapula and the serratus upwardly rotates the scapula. The subscapularis internally rotates the shoulder and the infraspinatus is responsible for depressing the humerus to prevent superior translation and for providing an antagonistic external rotation force (Moore and Dalley 2006).

The study of the normal shoulder also indicated that the subscapularis was active through the entire stroke at $26-71 \%$ of maximal voluntary isometric contraction (MVIC), with peak activity at late pull phase and early recovery and the serratus anterior had constant activity $20-45 \%$ of MVIC with increase at middle pull through and hand exit (Pink, Perry et al. 1991). These muscles are very important to the functioning of the shoulder during 
swimming and are very susceptible to fatigue. A study by Monod (Monod 1985) found that a muscle can only function at $15-20 \%$ of maximum voluntary contraction during sustained activity, or fatigue will occur. The subscapularis and serratus anterior are maintained in a contraction above the fatigue limit and are susceptible to fatigue, even in the healthy shoulder. One study in swimmers showed decreased scapular upward rotation in swimmers with impingement only after fatigue, which can be attributed to the decreased functioning of the serratus as it fatigues ( $\mathrm{Su}$, Johnson et al. 2004). The fatigued muscles can create altered scapular kinematics that lead to injury and are responsible for much of the shoulder pain in swimmers.

\section{Muscle Activity in the Painful Shoulder}

In a study done by Scovazzo et al (Scovazzo, Browne et al. 1991), the muscle activity of the same 12 muscles from the study by Pink et al (Pink, Perry et al. 1991) on normal shoulder, were recorded in individuals who had shoulder pain. The results are extremely useful in determining the needs of an injured swimmer. During hand entry there was less activity in the rhomboids, upper trapezius and anterior and middle deltoid. This altered muscle activity caused the hand to enter the water more lateral from the midline and with the humerus closer to the water than asymptomatic swimmers. The decreased anterior and middle deltoid activity causes dropped elbow, which coaches can recognize as a sign of injury, fatigue, or laziness during practice (McMaster 1999). During the pulling phase, there was less serratus anterior activity, accompanied by more activity in the rhomboids. The rhomboids substitutes for the fatigued serratus anterior, causing retraction of the scapula instead of upwardly rotating the scapula as well as maintaining joint congruency (Scovazzo, Browne et al. 1991). This alteration in the muscle activity will cause a change in the ability to 
propel the body and generate maximal force production. During hand exit, the anterior and middle deltoids had significantly less activity, while the infraspinatus has an increased amount of activity. The infraspinatus activity is also increased during the recovery phase, while the subscapularis activity is decreased. The infraspinatus is more active in order to promote external rotation and prevent or lessen impingement due to internal rotation. Through the entire stroke, the serratus anterior shows decreased activation patterns, which limits the propulsive motion that the serratus anterior can contribute. More importantly, the upward rotation usually created by the serratus anterior prevents impingement of the structures in the subacromial space as well as maintains joint congruency of the scapula and humerus (Pink, Perry et al. 1991; Wadsworth and Bullock-Saxton 1997). Without this movement, impingement is likely to occur due to compression of the structures in the subacromial space and instability due to an increased amount of space between the glenoid and humerus (Wadsworth and Bullock-Saxton 1997).

\section{Detraining in Swimmers}

A typical competitive swimming season for elite swimmers involves approximately 4-5 months of intensive training, followed by 1-2 months of taper leading up to the major competitions of the year (Stewart and Hopkins 2000). Because of the frequency of training, duration of the season and intensity of the workout, tremendous stress is placed on the shoulder, often leading to pain from shoulder impingement. While rest is often beneficial to the injured athlete, detraining can occur very quickly in the swimmer. After one week of inactivity, a $50 \%$ decline in the respiratory capacity of the deltoid muscle has been found, in addition to a significant rise in lactate concentration in the blood (Costill, Fink et al. 1985). Another study found that no swimming resulted in an $8 \%$ decrease in swim power, with 
progressive decreases each additional week of inactivity, as well as a significant increase in blood lactate levels (Neufer 1989). When training was decreased to only 1 day of training per week, a significant increase in stroke rate and significant decrease in stroke distance was found over a 4-week period (Neufer 1989). The tremendous amount of training that swimmers perform to get physiological adaptations of their muscles can be lost in as little as 1 week of inactivity, which most likely is recommended to the athlete because of a shoulder injury. Because of the tremendous repercussions of inactivity on the swimmer, preventing shoulder injury is paramount to their success. By understanding the demands of swimming as well as the physical profile of swimmers, a successful prevention program can be designed. Although prevention programs are prominent in other overhead sports such as baseball and softball, little research has been conducted on the efficacy of a program for preventing shoulder injuries in competitive swimmers.

\section{Intervention programs in Swimmers}

A study by Swanik et al (Swanik, Swanik et al. 2002) implemented a 6-week functional training program in 26 intercollegiate swimmers to determine its effects on shoulder strength and incidence of shoulder pain. The program included 7 exercises that were performed 3 days a week for 6 consecutive weeks. The exercise program included: internal and external rotation at $90^{\circ}$ with tubing, horizontal abduction/flexion and horizontal adduction/extension with tubing, external rotation with $90^{\circ}$ and $120^{\circ}$ abduction prone with free weights and push up plus. After 6 weeks, participants reported significantly less number of incidences of shoulder pain compared to a control group, despite finding no significant changes in strength. The treatment group averaged 1.8 incidences of shoulder pain while the control group averaged 4.6. The authors attributed the decreased incidence of shoulder pain 
to neuromuscular adaptation. The authors hypothesize that although no strength increases were found, the muscles had improved efficiency and during functional movement were able to better stabilize the humeral head and scapula, preventing increased contact with the rotator cuff tendons due to a decreased subacromial space width. This study shows that introducing a prevention program can produce significant impact on the frequency of shoulder pain. A limitation of this study was that the study did not contain exercises to specifically strengthen the scapular stabilizers, stretch the pectoralis major and minor, latissimus dorsi and posterior capsule, or increase muscular endurance of the internal rotators. In addition, this study did not measure the effect of the exercise program on scapular kinematics. Altered scapular kinematics has been found to be associated with shoulder pain and the decreased reported incidences of pain may be due to improved movement patterns (Lukasiewicz, McClure et al. 1999; Ludewig and Cook 2000; Ludewig and Reynolds 2009).

Other intervention programs have been implemented to address many of the strength and range of motion imbalances that swimmers possess. In a study by Kluemper et al (Kluemper, Uhl et al. 2006) a 6-week stretching and strengthening program was implemented on competitive swimmers for correction of forward shoulder posture. A double square was used to identify swimmers with forward shoulder posture and subjects were randomly assigned to treatment and control groups. The treatment group performed scapular retraction, external rotation and shoulder flexion using resistive tubing, as well as stretching the pectoralis minor while laying over a foam roller and the pectoralis major with the individuals hand linked behind their head with the a partner applying overpressure. After the 6 weeks, a significant improvement in posture was found in the treatment group, with these individuals having decreased forward posture. The positive results from this study show that compliance 
with a stretching and strengthening program can improve posture. Although this study demonstrated the positive effect of the intervention program on forward shoulder posture, it did not investigate strength, range of motion, or scapular kinematic changes that may have occurred due to the intervention program. While posture is theorized to be a cause of shoulder injury, little empirical evidence exists to support this idea. In addition, no consistent postural deviations have been found in subjects with impingement syndrome or for any other shoulder pain (Lewis, Green et al. 2005). The information regarding strength and scapular kinematic changes would allow the clinician to evaluate the effectiveness of implementing the program in swimmers to address their known strength and range of motion deficits, in addition to improving their posture.

A study by Wang et al (Wang, McClure et al. 1999) implemented a strengthening and stretching program on asymptomatic participants with forward shoulder posture and evaluated its effect on three-dimensional scapular kinematics and strength. Participants performed scapular retraction, shoulder shrugging, shoulder abduction, shoulder external rotation and corner stretching using resistive tubing three times per week. After the intervention, a significant increase in isometric strength for external rotation, internal rotation and horizontal abduction occurred. However, no significant difference was found in threedimensional scapular position at rest after the 6 weeks, although change in scapulohumeral rhythm was found, when the arm was actively elevated to 90 . The scapula was found to be less upwardly rotated, superiorly translated and more internally rotated, indicating the increased contribution from the glenohumeral joint to the elevation movement. After the exercise program, the scapular stabilizing muscles were better able to stabilize the scapula on the thorax and help to prevent impingement. This study was performed using healthy 
individuals, but they were not collegiate athletes. The demands of a collegiate athlete are very rigorous and the results of a study that uses healthy individuals may not be directly applicable to the collegiate swimmer who swims 11 practices per week, lifts 4 times per week and completes dryland 3 times per week.

McClure et al (McClure, Bialker et al. 2004) developed an intervention program designed for individuals with shoulder impingement syndrome. These individuals were given a 6-week exercise program to address strength and range of motion deficits. The exercise program included: strengthening with resistive tubing by performing shoulder external rotation at the side, shoulder internal rotation at the side, shoulder extension, shoulder abduction, shoulder flexion, scapular retraction and shoulder ER with arm at $45^{\circ}$ abduction and a stretching program including a internal rotation towel stretch, cross body stretch, upper thoracic extension stretch, doorway pectoral stretch, shoulder flexion stretch and shoulder external rotation stretch. After 6 weeks, passive internal rotation and external rotation range of motion and abduction and internal force improved. Subjects also reported improvements in pain and satisfaction related to ability to function, based on self-report measures from the University of Pennsylvania shoulder scale. Because improvement in symptoms were found with out improvement in scapular kinematics, the authors suggest that improving scapular kinematics may not be the primary mechanism of improving symptoms and that not all individuals with impingement also have altered scapular kinematics.

\section{EMG analysis of shoulder strengthening exercises}

Electromyographic (EMG) studies focusing on the shoulder musculature while performing rehabilitation exercises can provide valuable information about the effectiveness of the exercises in strengthening the desired muscles. 
Ballantyne et al (Ballantyne, O'Hare et al. 1993) performed a study to determine if shoulder musculature activation patterns differed between healthy individuals and those with shoulder pathology while performing common shoulder rehabilitation studies. Fine wire EMG electrodes were used to evaluate the muscle activity in the supraspinatus, infraspinatus, teres minor and lower trapezius while performing prone lateral rotation, sidelying lateral rotation, and empty can exercises. This study found that both healthy and painful individuals exhibited a similar activation pattern across all four exercises. Similarly, studies by Kibler et al (Kibler, Sciascia et al. 2008) and Ludewig et al (Ludewig, Hoff et al. 2004) demonstrated no difference in muscle activation pattern during exercises between individuals with and without shoulder pain. Significant differences in muscle activation levels based on the exercise that was performed existed regardless of the injury status, but all 4 muscles were activated during every exercise. Prone and sidelying rotation were found to activate the teres minor and infraspinatus equally, but prone sidelying rotation activated the lower trapezius more effectively. The supraspinatus was most effectively activated by the empty can exercise.

Cordasco et al (Cordasco, Wolfe et al. 1996) evaluated the EMG activity during a shoulder rehabilitation program using a medicine ball. Subjects performed a two handed overhead throw while surface EMG evaluated the activation of 10 shoulder muscles during the cocking, acceleration and deceleration phases. This study observed that the most activation of all of the muscles occurred during the acceleration phase of the throw, with the upper trapezius, subscapularis, pectoralis major, anterior and middle deltoid, and supraspinatus maintaining a high level of activity. During the deceleration phase, the upper trapezius was the only muscle to maintain a high level of activity, but eight other muscles 
had moderate levels of activation. This study evaluates the use of functional rehabilitation exercises and shows that they are effective in activating multiple muscles simultaneously.

Moseley et al (Moseley, Jobe et al. 1992) aimed to find the exercises that most effectively activated the scapular muscles. Healthy subjects had indwelling EMG electrodes in their upper, middle and lower trapezius, levator scapulae, rhomboids, pectoralis minor, and serratus anterior. Sixteen shoulder exercises were performed and exercises that had greater than $50 \%$ of pretest maximal manual test values were classified as optimal. The twelve shoulder exercises that were determined to be optimal were: rowing, military press, horizontal abducting in neutral and humeral external rotation, scaption, abduction, prone extension, flexion, shrug, scaption, push-up with a plus, press-up and push-up with hands apart. The top 4 exercises overall were scaption, rowing, push-up with a plus, and press-up.

Cools et al (Cools, Dewitte et al. 2007) performed a study to determine a rehabilitation program that utilized optimal scapular muscle balance. Optimal scapular muscle balance occurred when the ratios between the upper trapezius:lower trapezius, upper trapezius:middle trapezius and upper trapezius:serratus anterior were small. This means that when there is high activation of the lower trapezius, there is low activation of the upper trapezius. The small ratio is important in determining exercises that strengthen muscles that are typically underactive, while creating minimal activation in muscles that tend to be overactive. Sidelying external rotation, sidelying forward flexion, prone horizontal abduction and prone extension were found to be the exercises with the lowest upper trapezius:lower trapezius and upper trapezius:middle trapezius ratio. No shoulder exercises included in this study were found to optimize the upper trapezius:serratus anterior ratio.

Oyama and Myers (Oyama and Myers In Review) evaluated scapular kinematics and 
muscle activations during 20 common rehabilitation exercises for scapular and shoulder stabilizers. The goal of this study was to identify exercises that result in the greatest scapular upward rotation, external rotation, posterior tipping and retraction. The following exercises were determined to have increased scapular stabilizer activation and desired scapular kinematics: Wand flexion, Ys, Ws, ER @ 90 $90^{\circ}$, No Money, Throwing motion, serratus punches, and diagonal flexion/extension.

Hintermeister et al (Hintermeister, Lange et al. 1998) measured EMG activity during shoulder rehabilitation exercises with elastic tubing. Healthy subjects performed external and internal rotation, forward punch, shoulder shrug, and seated rowing with a narrow, middle and wide grip while fine-wire intramuscular electrodes and surface electrodes evaluated the activation of shoulder musculature. During the wide-grip seated rowing exercise, which is similar to the $\mathrm{W}$ exercise, activated the supraspinatus, infraspinatus, and trapezius were activated throughout the range of motion. The serratus anterior was most active during the forward punch exercise, and became more active as the force increased at the end range of motion. As a whole, these exercises were determined to effectively activate the scapular stabilizing and rotator cuff musculature.

A study by Myers et al (Myers, Pasquale et al. 2005) evaluated the 12 most common rubber tubing exercises commonly performed during an on-field injury prevention programs of pitchers. The exercises were evaluated for activation of muscles important in the throwing motion, including the rotator cuff, scapular stabilizers and humeral movers. The 12 most common rubber tubing exercises included shoulder extension, shoulder flexion, internal humeral rotation at $0^{\circ}$ of abduction, external humeral rotation at $0^{\circ}$ of abduction, internal humeral rotation at $90^{\circ}$ of abduction, external humeral rotation at $90^{\circ}$ of abduction, scapular 
punches, throwing acceleration and throwing deceleration. The exercises that are recommended from this study were based on the number of muscles that were at least moderately activated, which was considered at least $20 \%$ of the measured maximum voluntary isometric contraction. The 7 recommended exercises were shoulder extension, shoulder flexion, throwing acceleration, throwing deceleration, external rotation at $90^{\circ}$ of abduction, scapular punches and the low row. These exercises strengthen both shoulder muscles as well as scapular stabilizers and can easily be performed on the field. Pezullo et al (Pezzullo, Karas et al. 1995) also investigated resistive tubing exercises for overhead athletes and found that internal rotation and external rotation at $90^{\circ}$ and diagonal pattern acceleration and deceleration are the most effective exercises to perform.

\section{Stretching Programs}

Posterior shoulder tightness has been identified as one of the causes of impingement and several studies have been conducted to determine the most effective stretching program for improvements in the associated internal rotation loss. In a study using male baseball players and non-throwing control subjects, baseball players had a significant increase in posterior shoulder motion and internal rotation after completing $2 \times 30$ seconds of the sleeper stretch (Laudner, Sipes et al. 2008). Lintner et al (Lintner, Mayol et al. 2007) conducted a study to determine the long term effects of a posterior capsule stretching program. Internal rotation in baseball pitchers who reported performing a stretching program for 3 or more years was significantly greater compared to pitchers who reported less than 3 years involvement in a stretching program. McClure et al (McClure, Balaicuis et al. 2007) conducted a study to determine the most effective stretch for the posterior shoulder structures. Subjects were split into a sleeper stretch group, cross body stretch group and a 
control group. Subjects were asked to perform the stretching exercise once daily for 5 repetitions, holding each stretch for 30 seconds. This study found that the cross body stretch was the most effective stretch for individuals with limited shoulder internal rotation range of motion. Most recently, a study using strain values in cadaver models found that the most effective technique for stretching the middle and lower posterior capsule was with $30^{\circ}$ of elevation in the scapular plane with internal rotation and $30^{\circ}$ extension with internal rotation of the shoulder (Izumi, Aoki et al. 2008)

\section{Instrumentation}

\section{Handheld Dynamometer}

A hand-held dynamometer (HHD) will be used to collect isometric strength data (Model: Lafeyette Manual Muscle Test System Model number 01163). HHDs are an efficient and convenient tool for measuring isometric strength. HHDs have been shown to be reliable and valid measures for assessing muscle strength, as long as the assessor's strength is stronger than the muscle group being tested (Stratford and Balsor 1994). Shoulder and scapular stabilizing musculature strength measurements using a hand-held dynamometer have been proven to be reliable and valid (Stratford and Balsor 1994; Leggin, Neuman et al. 1996; Hayes, Walton et al. 2002; McClure, Balaicuis et al. 2007). A pilot study was conducted to determine the intersession reliability of a HHD to measure shoulder and scapular stabilizer strength. These results are included in Table $\mathbf{1}$.

\section{Motion Monitor electromagnetic tracking device}

The Motion Monitor electromagnetic tracking device (Innovative Sports Training Inc, Chicago, Ill) will be used to collect scapular kinematics. The Motion Monitor 
electromagnetic tracking device integrated with the Motion Monitor software will use the data acquired through electromagnetic receivers for the calculation of receiver position and orientation relative to the electromagnetic transmitter. The electromagnetic tracking device consists of a transmitter that creates an electromagnetic field and receivers that detect the electromagnetic field. A mid-range direct current transmitter and four receivers will be used for data collection in this study. Karduna et al (Karduna, McClure et al. 2001) validated this method of measuring scapular kinematics against a measurement using a direct insertion of a bone pin in to the scapula. Participants in the study had receivers set on designated locations on the scapula as the non-invasive technique and pins were inserted for the bone assessment, which was done to validate this method. The study found that with careful placement of the receivers, an accurate and valid representation of scapular kinematics can be found (Karduna, McClure et al. 2001). Kinematics will be sampled at $100 \mathrm{~Hz}$. This measure has been found to be reliable and valid in measuring scapular kinematics (Karduna, McClure et al. 2001; Myers, Laudner et al. 2005; Thigpen, Gross et al. 2005) Reliability and precision of the measurement of scapular kinematics using an electromagnetic tracking device are listed in Table 2 (Myers, Jolly et al. 2006).

\section{SANE and Dash}

The Single Assessment Numeric Evaluation (SANE) and sports module from the Disabilities of the Arm, Shoulder, and Hand (DASH) are questionnaires used to obtain information about an individual's satisfaction with his/her shoulder and a subjective measure of shoulder functioning (Appendix 1). The SANE is measurement tool that asks subjects to rate his/her shoulder on a scale of 1-100. The SANE was found to be highly correlated with the modified Lysholm score, which has been found to be valid (Lysholm and Gillquist 1982; 
Tegner and Lysholm 1985; Williams, Taylor et al. 2000). The sports module of the DASH is a subjective measure of shoulder functioning during athletic performance that individual's rank on a scale of 1-5. The DASH has been found to be a reliable and valid assessment tool (Williams, Gangel et al. 1999; Williams, Taylor et al. 2000; Huisstede, Feleus et al. 2009).

\section{Summary}

Competitive swimmers place a tremendous amount of stress on their shoulders as this is the primary mechanism for propelling their bodies through the water (Hawley and Williams 1991). Competitive swimmers train approximately 10,000-14,000 meters per day, 6-7 times per week, which correlates to 16,000 shoulder revolutions per week (Stocker, Pink et al. 1995; Pink and Tibone 2000). Because of the significant number of shoulder revolutions performed during participation, shoulder pain has become commonplace in competitive swimming, comprising at least $55 \%$ of all injuries and affecting between $45-87 \%$ of all swimmers during their careers (Johnson 1988; Beach, Whitney et al. 1992; Stocker, Pink et al. 1995; McFarland and Wasik 1996; Urwin, Symmons et al. 1998; Pink and Tibone 2000; Bongers 2001). Research has found that swimmers have altered patterns of shoulder muscle strength, range of motion and scapular kinematics, which may further predispose them to shoulder injury. When shoulder pain does occur, rest is typically given to swimmer to all for recovery. While rest may help the shoulder pain, detraining can quickly occur in swimmers. After one week of inactivity, a $50 \%$ decline in the respiratory capacity of the deltoid muscle, $8 \%$ decrease in swim power, decreased stroke distance, and significant rise in lactate concentration in the blood was found (Costill, Fink et al. 1985; Neufer 1989). Although shoulder injury prevention programs have become the norm in overhead sports like 
baseball and softball, an effective prevention program has yet to be established for swimmers. Therefore, the purpose of this study is to determine an effectiveness of the prevention program for shoulder and scapular stabilizer strengthening and improving scapular kinematics in division 1 collegiate swimmers which could potentially decrease shoulder pain and detraining that may occur from rest due to injury. 


\section{Chapter III METHODOLOGY}

\section{Population and Recruitment}

Subjects were recruited from a university population of NCAA Division I competitive swimmers at The University of North Carolina at Chapel Hill. Subjects were both males and females between the ages of 18-25 years old. The primary researcher met with all potential subjects and explained the research project. Individuals interested in participating provided informed consent and were enrolled.

\section{Subject Inclusion Criterion}

Subjects were included in the study if they met the following criteria:

- NCAA division I swimmers

- Participated in swimming for at least 30 minutes per day for 4 days per week.

- Participated in all team weight lifting sessions.

\section{Subject Exclusion Criteria}

Subjects were excluded from the study if:

- They were being treated for shoulder pain

- They developed shoulder pain during the course of the intervention program

- They did not complete 15 of the 18 training sessions 


\section{Instrumentation}

\section{Hand-held dynamometer}

A hand-held dynamometer (HHD) was used to collect isometric strength data (Model: Lafeyette Manual Muscle Test System Model number 01163). HHDs are an efficient and convenient tool for measuring isometric strength. HHDs have been shown to be reliable and valid measures for assessing muscle strength, as long as the assessor's strength is stronger than the muscle group being tested (Stratford and Balsor 1994). Shoulder and scapular stabilizing musculature strength measurements using a hand-held dynamometer have been proven to be reliable and valid (Stratford and Balsor 1994; Leggin, Neuman et al. 1996; Hayes, Walton et al. 2002; McClure, Balaicuis et al. 2007). In-house reliability data are listed in Table 1.

\section{Electromagnetic tracking device}

The Motion Monitor electromagnetic tracking device (Innovative Sports Training Inc, Chicago, Ill) was used to collect scapular kinematics. The Motion Monitor electromagnetic tracking device integrated with the Motion Monitor software was used for the data acquired through electromagnetic receivers for the calculation of receiver position and orientation relative to the electromagnetic transmitter. A mid-range direct current transmitter and four receivers were used for data collection. Scapular kinematics were sampled at $100 \mathrm{~Hz}$. This measure has been found to be reliable and valid in measuring scapular kinematics (Karduna, McClure et al. 2001; Myers, Laudner et al. 2005; Thigpen, Gross et al. 2005). Reliability and precision of the measurement of scapular kinematics using an electromagnetic tracking device are listed in Table 2 (Myers, Jolly et al. 2006). 


\section{Research Design}

A randomized control trial with an intervention and control group was used in this study. The subjects reported for a pretest screening. During the pretest screening researchers took measurements of strength and scapular kinematics. Following the pretest screenings, the participants were assigned subject numbers and stratified by sex, to ensure an equal number of males and females in each group. The stratified subject numbers were randomized in Excel. The first half of the randomized subject numbers were assigned to be in the intervention group and the rest were placed in the control group. This method ensured randomization and that an equal number of males and females were in each group. The treatment group underwent an extensive introduction to the intervention program.

Participants in the intervention and control group attended the same team dry land workouts, weights and practices. In addition, participants in the intervention group performed the shoulder strengthening/stretching program after practice, while control participants did not perform any additional exercises or stretching. Following the 6-week intervention period, all subjects were retested. Researchers performing the measurements of strength were blinded to group assignment to prevent assessor bias. These researchers were volunteers that were not affiliated with the swimming team and therefore were not present at any of the training sessions.

\section{Procedures}

When the subject reported for the initial screening, he/she was introduced to the experiment and then read and signed a consent form approved by the University of North Carolina at Chapel Hill Institutional Review Board. The subject then underwent the preintervention testing, which included assessments of shoulder and scapular stabilizer strength 
and scapular kinematics on the dominant side and subjective measures of shoulder functioning. Details of each procedure are discussed below.

\section{Pre-Intervention Testing}

\section{Shoulder and Scapular Stabilizer Strength}

All manual muscle testing was performed using a procedure described by Kendall (Kendall, McCreary et al. 2005). For each strength test, the subject was placed in the appropriate position and instructed to hold the position. The researcher pushed hard enough with a hand held dynamometer to break the position and then recorded the peak force. Each position was tested 3 times and the average of the three trials was used for statistical analyses.

Shoulder flexion strength: The subject sat on the table with the dominant side shoulder flexed to $90^{\circ}$ with the elbow extended and the palm in a pronated position. The examiner applied pressure to the arm slightly above the elbow. This manual muscle test assessed the strength of the shoulder flexors, which include the anterior deltoid, coracobrachialis, biceps brachii and the upper portion of the pectoralis major. (Figure 1)

Shoulder extension strength: The subject lay prone on the table. The dominant arm was extended $20^{\circ}$, while a stabilizing hand applied counter-pressure laterally on the pelvis. The examiner applied pressure against the arm slightly above the elbow, in the direction of the table. This manual muscle test best isolated the latissimus dorsi. (Figure 2)

Shoulder external/internal rotation strength: The subject lay prone on the table with $90^{\circ}$ of shoulder abduction and elbow flexion. The examiner stabilized the elbow to isolate the rotational motion at the glenohumeral joint. To assess external rotation strength, the examiner applied a force on the back of the wrist to rotate the forearm toward the foot while the subject 
resisted the force. Internal rotation strength was assessed in a similar manner. The investigator applied a force on the front of the wrist and rotated the forearm toward the head while the subject resisted the force. (Figure 3 and 4)

Shoulder abduction strength: The subject was seated on a chair with the arm elevated to his/her shoulder height with $90^{\circ}$ of shoulder abduction (frontal plane). The examiner applied a force slightly above the elbow to push the arm down toward the floor while the subject resisted the force. (Figure 5)

Shoulder adduction strength: The subject was seated on a chair with $35^{\circ}$ of shoulder abduction and the elbow extended with the palm pronated. The examiner pressed on the medial aspect of the arm slightly above the elbow as the examiner tried to abduct the arm. This manual muscle test assessed the strength of the shoulder adductors, which includes the pectoralis major, teres major and latissimus dorsi. (Figure 6)

Scapular Retraction (T): The subject lay prone with the arm in $90^{\circ}$ of abduction and in lateral rotation. The examiner placed the stabilizing hand on the opposite scapula to prevent trunk rotation and pressed on the arm slightly above the elbow in a downward direction toward the table. This manual muscle test assessed the strength of the middle trapezius.

\section{(Figure 7)}

Scapular Retraction with upward rotation strength $(Y)$ : The subject lay prone with the arm approximately $45^{\circ}$ higher than shoulder height, in line with the lower fibers of the trapezius, with lateral rotation and elevation of the arm. The examiner placed the stabilizing hand below the opposite scapula in order to prevent trunk rotation and pressed against the forearm in a downward direction toward the table. This manual muscle test assessed the strength of the lower trapezius. (Figure 8) 
Scapular retraction with downward rotation strength $(W)$ : The subject lay prone with the dominant arm in humeral adduction, extension and slight lateral rotation with the elbow flexed. The examiner applied pressure with one hand against the subjects arm slightly above the elbow in the direction of abducting the scapula. This manual muscle test is aimed to best isolate the rhomboids. (Figure 9)

\section{Scapular Kinematics}

The subjects were fitted with electromagnetic tracking receivers that were used with the Motion Monitor system to track scapular kinematics. The male subjects removed their shirts and female subjects wore a tank top to make marker placement more accurate and secure. A total of 4 receivers were used for the scapular kinematics assessment (Figure 10). Three of the receivers were placed on the spinous process of the seventh cervical spine (C7), the flat portion of the acromion process of the dominant arm and mid-shaft of the posterior humerus of the dominant arm (Myers, Laudner et al. 2005). The receivers were secured to the skin using double-sided tape, pre-wrap, athletic tape and a velcro belt to minimize movement between the skin and receiver.

The fourth receiver was attached to the stylus and was used to digitize the anatomical landmarks of the upper arm, scapula, and thorax. The anatomical landmarks digitized were: the eighth thoracic vertebrae, xiphoid process, seventh cervical vertebrae, jugular notch, sternoclavicular (SC) joint, acromioclavicular (AC) joint, medial scapular border where it intersects with the scapular spine, inferior angle of the scapula, medial epicondyle, and lateral epicondyle (Table 3). The SC joint on the thorax segment and the AC joint on the scapular segment were digitized to be used to calculate the indirect clavicular kinematics (Ludewig, Behrens et al. 2004; Myers, Laudner et al. 2005), which represented scapular position. The 
glenohumeral joint center was estimated as the point that moves the least with respect to the scapula as the humerus was passively moved through several short arcs (Meskers, Vermeulen et al. 1998). The local coordinate systems for the thorax, scapula, and humerus were constructed based on the digitization of these anatomical landmarks (Table 4). The position and orientation of the scapula relative to the thorax were calculated based on these local coordinate systems (Myers, Laudner et al. 2005).

After all of the points were digitized, the subjects were instructed on a humeral elevation task in the scapular plane. Guidance for this elevation motion was provided by means of a PVC pipe guide to keep the subjects' arms in the scapular plane and tape placed on the floor in the scapular plane to provide additional guidance. The scapular plane was defined as $30^{\circ}$ anterior to the frontal plane for this study. Shoulder rotation was in the neutral position and the subject was instructed to maintain this position by keeping their thumbs pointing towards the ceiling. Data were only analyzed from $0^{\circ}$ elevation (arm at side) to $120^{\circ}$ elevation. Data were not be analyzed above $120^{\circ}$ as there was a possibility that skin movement may cause inaccurate values of scapular kinematics (Karduna, McClure et al. 2001). The subject performed 15 elevations at a rate of 4 seconds per repetition with 2 seconds of raising the arm and 2 seconds of lowering the arm (Karduna, McClure et al. 2000; McClure, Bialker et al. 2004; Thigpen, Padua et al. 2006).

\section{SANE and DASH}

Before the testing session began, all subjects were asked to fill out a form related to shoulder satisfaction and functioning during activity. This questionnaire included a Single Assessment Numeric Evaluation (SANE) of the Shoulder and the Sports Module from the Disabilities of the Arm, Shoulder, and Hand (DASH) (Appendix 1). The SANE and DASH 
have been shown to be reliable and valid methods for assessing shoulder functioning (Williams, Gangel et al. 1999; Williams, Taylor et al. 2000; Huisstede, Feleus et al. 2009). All subjects also filled out this form at the post-intervention testing.

\section{Intervention Program}

Subjects in the treatment group performed the intervention program after practice three days per week. The principal investigator monitored training sessions to ensure that subjects were performing the exercises correctly and track compliance with a log sheet. Subjects in the control group were allowed to leave the pool deck to shower while the treatment group was completing the intervention program. The intervention program included: shoulder flexion, shoulder extension, shoulder external rotation at $90^{\circ}$ abduction, shoulder internal rotation at $90^{\circ}$ abduction, low rows, D2 pattern acceleration, D2 pattern deceleration, scapular punches, Ys, Ts and Ws (Figures 11-21). Subjects performed 2 sets of 15 repetitions of each of the exercises 3 times per week for six weeks. These exercises have been shown to be the most effective resistive tubing exercises for activating the muscles that have been found to be weak in swimmers (Table 5) (Cordasco, Wolfe et al. 1996; Hintermeister, Lange et al. 1998; Ekstrom, Donatelli et al. 2003; Myers, Pasquale et al. 2005; Cools, Declercq et al. 2007). Subjects also performed 2 stretching exercises: the sleeper stretch and the corner stretch, which have been shown to be effective in improving range of motion and allowing for optimal functioning of the shoulder musculature (Figures 22-23) (Borstad and Ludewig 2006; McClure, Balaicuis et al. 2007). Subjects completed 2 sets of 30 seconds for each of the stretches.

At the first session, all subjects were given different levels of Thera-band ${ }^{\circledR}$ rubber tubing exercise bands (Hygenic Corp, Akron, Ohio) to determine the band with appropriate 
resistance. These bands are color-coded, with each color representing a different resistance. The subjects performed 5 repetitions of each exercise with different levels of resistive tubing in order to estimate which resistance was appropriate (Kluemper, Uhl et al. 2006). Subjects were instructed to choose a level of resistance that was challenging, but that the exercises could be completed. Feedback from the subject and observation of proper form were used to determine if the appropriate resistance level had been chosen. If the level chosen was too difficult, lower level resistive tubing was given and higher level resistive tubing was given if it was too easy. Subjects were instructed to contact the researcher if the chosen resistive tubing becomes too easy during the program. After two and four weeks, all subjects were reevaluated to ensure that they were using the appropriate resistance.

\section{Strengthening Exercises}

Shoulder Flexion: The subject stood facing away from the wall with the elbow extended and the forearm in a neutral position (thumb pointing up). The subject moved his/her arm into full shoulder flexion and then returned to the starting position while keeping both elbows extended and forearm in a neutral position. The resistive tubing was secured to the stable base at a height equal to the height of each subject's fingertips from the ground (Myers, Pasquale et al. 2005). (Figure 11)

Shoulder Extension: The subject stood facing towards the wall with the elbow extended, the forearm in neutral position and the shoulder flexed to $90^{\circ}$. The subject moved the shoulder toward maximum extension and then returned to the starting position while maintaining full elbow extension and the forearm in a neutral position. The resistive tubing was secured to the stable base at the height of each subject's fingertips with the shoulder 
fully flexed in a standing position (Myers, Pasquale et al. 2005). (Figure 12)

Shoulder External Rotation at $90^{\circ}$ : The subject stood facing the wall with the shoulder abducted and elbow flexed $90^{\circ}$. The subject began in full internal rotation, moved the shoulder into full external rotation and then returned to the starting position. The resistive tubing was secured to the stable base at a height equal to the height of each subject's fingertips from the ground (Myers, Pasquale et al. 2005). (Figure 13)

Shoulder Internal Rotation at $90^{\circ}$ : The subject stood facing away from the wall with the shoulder abducted and the elbow flexed to $90^{\circ}$. The subject began in full external rotation, moved into full internal rotation and then returned to the starting position. The resistive tubing was secured to the stable base at the height of each subject's fingertips with the shoulder fully flexed in a standing position (Myers, Pasquale et al. 2005). (Figure 14)

Low Rows: The subject stood facing the stable base with the elbow extended and the scapula protracted. The subject moved the scapula into full retraction with accompanying elbow flexion and then returned to the starting position. The resistive tubing was secured to the stable base at a height equal to the height of each subject's fingertips from the ground (Myers, Pasquale et al. 2005). (Figure 15)

Throwing Acceleration: The subject stood facing away from the wall with the shoulder abducted and elbowed flexed to $90^{\circ}$. The subject began with the shoulder in full external rotation and moved the arm across the body similar to the acceleration phase of throwing and then returned to the staring position. The resistive tubing was secured to the stable base at the height of each subject's fingertips with the shoulder fully flexed in a standing position (Myers, Pasquale et al. 2005). (Figure 16)

Throwing Deceleration: The subject stood facing the wall with the shoulder in $30^{\circ}$ of 
flexion and then pulled the tubing back so the shoulder was in full extension and scapular retraction. At full shoulder extension, the subject moved their shoulder to $90^{\circ}$ each of shoulder external rotation, shoulder abduction and elbow flexion. The exercise was then finished with the subject eccentrically controlling the tubing as the arm returned to the original start position. The resistive tubing was secured to the stable base at a height equal to the height of each subject's fingertips from the ground (Myers, Pasquale et al. 2005).

\section{(Figure 17)}

$Y_{s}$ : The subject started with his/her shoulders flexed to $90^{\circ}$ and the elbows fully extended with the palms down facing the stable base. The subject flexed the shoulders to $180^{\circ}$ and then returned to the start position, focusing on a controlled return. The resistive tubing was secured to the stable base at a height equal to each subject's fingertips from the ground (Ekstrom, Donatelli et al. 2003). (Figure 18)

$T_{s}$ : The subject started with his/her shoulders flexed to $90^{\circ}$ and the elbows fully extended with the palms down facing the stable base. The subject horizontally abducted the shoulders to $180^{\circ}$ and then returned to the start position, focusing on a controlled return. The resistive tubing was secured to the stable base at a height equal to each subject's fingertips from the ground (Ekstrom, Donatelli et al. 2003). (Figure 19)

Ws: The subject started with his/her shoulders flexed to $90^{\circ}$ and the elbows fully extended with the palms down facing the stable base. The subject abducted the shoulders $25^{\circ}$, flexed the elbows $90^{\circ}$ and retracted the shoulders and then returned to the start position, focusing on a controlled return. The resistive tubing was secured to the stable base at a height equal to each subject's fingertips from the ground (Ekstrom, Donatelli et al. 2003). (Figure 
Scapular Punches: The subject faced away from the stable base with the elbow flexed $45^{\circ}$, the arm abducted $60^{\circ}$, and the shoulder internally rotated $45^{\circ}$. The subject moved into maximum scapular protraction by horizontally flexing the humerus in an arc mimicking a hug. Once the subject's hand touched together, he/she returned to the starting position. The resistive tubing was secured to the stable base at the level of the shoulders (Decker, Hintermeister et al. 1999). (Figure 21)

\section{Stretching Exercises}

Sleeper Stretch: The subject laid on the side to be stretched and elevated the humerus to $90^{\circ}$ on the support surface, passively internally rotating the humerus with the opposite arm. The stretch was held for 30 seconds and repeated 2 times (McClure, Balaicuis et al. 2007).

\section{(Figure 22)}

Corner Stretch: The subject stood with the humerus abducted to $90^{\circ}$, the elbow flexed to $90^{\circ}$, and the palm on a flat surface. The subject rotated the trunk away from the elevated arm thereby increasing the horizontal abduction angle at the shoulder. The stretch was held for 30 seconds and repeated 2 times (Borstad and Ludewig 2006). (Figure 23)

\section{Post-Intervention Testing}

After the completion of the six-week intervention program, the subjects reported back to the Smith Center Athletic Training Clinic for follow-up testing. The procedures for the post-intervention testing were identical to those of the pretest protocol. 


\section{Data Reduction}

\section{Strength Data Reduction}

Strength data were recorded into Excel (version 12.1.5 for Macintosh). The mean of the three trials for each variable was calculated and normalized to body weight.

\section{Subjective Ranking Data Reduction}

A composite score for the Sports Module of the Disabilities of the Arm, Shoulder, and Hand was calculated based on the subject's responses (Solway, Beaton et al. 2002). The responses were averaged, creating a score out of five. This number was then transformed to a score out of 100 by subtracting one and multiplying by 25 . A higher score indicates greater disability.

DASH composite score $=[($ (sum on $n$ responses $)-1] \times 25$

\section{$\mathrm{n}$}

* $\mathrm{n}$ is equal to the number of completed responses

\section{Kinematic Data Reduction}

Raw scapular kinematic data were filtered with a low pass $10 \mathrm{~Hz}$ Butterworth filter. Receiver position and orientation data of the thoracic, scapular, and humeral receivers were transformed into a local coordinate system for each of the respective segments. The coordinate systems used were in accordance with recommendations from the International Society of Biomechanics (Table 3) (Wu, van der Helm et al. 2005). As the subject stood in anatomical position, the coordinate system for each segment was: vertical (y-axis), horizontal to the right (z-axis) and anterior (x-axis) (Figure 24). Orientation of the scapula was determined as rotation about the $y$-axis of the scapula (internal/external rotation), rotation about the z-axis of the scapula (upward/downward rotation) and rotation about the $\mathrm{x}$-axis of the scapula (anterior/posterior tipping)(Figure 25). 
Euler angles ( $\mathrm{Y}-\mathrm{X}-\mathrm{Z}$ order) were used to determine the scapular orientation with respect to the thorax and Euler angles (Y-X-Y order) were used to determine the position of the humerus relative to the thorax. The rotation sequence of the Euler angle was chosen from the recommendations of the International Shoulder Group (Wu, van der Helm et al. 2005). Based on these recommendations from the ISB, scapular movements in internal rotation, downward rotation and posterior tilt directions were indicated by the positive numbers $(\mathrm{Wu}$, van der Helm et al. 2005). Scapular upward rotation values were multiplied by -1 to make upward rotation a positive movement. This was done to make clinical interpretation easier. The clavicular kinematic measurements represent the scapular position relative to the clavicle and are not actual clavicular motions. The measurements consider clavicular kinematics were scapular protraction/retraction angle and scapular elevation/depression angle. The scapular protraction/retraction angle was calculated as the angle formed between the vector extending from the $\mathrm{SC}$ to the $\mathrm{AC}$ joints projected onto the transverse plane of the thorax and the frontal plane of the thorax and the scapular elevation/depression angle were calculated as the angle formed between the vector projected onto the frontal plane of the thorax and the transverse plane of the thorax (Wu, van der Helm et al. 2005). Mean scapular/clavicular orientations at $0^{\circ}, 30^{\circ}, 60^{\circ}, 90^{\circ}$ and $120^{\circ}$ of humeral elevation during the ascending phase of the elevation task was calculated for statistical analyses by averaging the values from the 15 repetitions of the elevation task. The first 3 repetitions and the last 2 repetitions were excluded from analysis to account for learning effects and fatigue. All calculations and data reduction were performed using Matlab software (The MathWorks Inc., Natick, Massachusetts).

\section{Statistical Analysis}

Statistical analyses were run using SPSS version 16.0 for Macintosh. Nine two-way 
ANOVAs with one within factor (session) and one between factor (group) were run to determine differences in normalized strengths between groups and sessions. Two two-way ANOVAs were run with one within factor (session) and one between factor (group) to assess the SANE and DASH ranking. Post hoc analysis with Bonferroni corrections were conducted to determine where the significant differences between groups and sessions existed. Five three-way mixed model ANOVAs with two within factors (session and angle) and one between factor (group) were used to examine the main effects and interactions of angle, groups, and time on the scapular kinematic variables. Huynh-Feldt correction was used whenever the assumption of sphericity was rejected. Post hoc analysis with Bonferroni corrections was conducted to determining where the differences occurred. Alpha levels were set a priori as $p=0.01$ to prevent errors as multiple t-tests were performed.

\section{Power Analysis}

The use of previous literature (Wang, McClure et al. 1999) has indicated that a sample size of 13 for each group was required to achieve a power of .80 with an alpha level of .05 (two tailed hypothesis test). This enabled possible differences in strength values to be observed between the treatment and the control group. 


\section{Summary of Research Questions}

\begin{tabular}{|c|c|c|c|c|}
\hline Question & Description & Data Source & Comparison & Method \\
\hline 1 & $\begin{array}{l}\text { What is the effectiveness of a } \\
\text { 6-week intervention program } \\
\text { in improving shoulder } \\
\text { strength? }\end{array}$ & $\begin{array}{l}\text { Average of three trials of } \\
\text { MMT break tests measured } \\
\text { with Handheld } \\
\text { Dynamometer and then } \\
\text { normalized to body weight. }\end{array}$ & $\begin{array}{l}\text { Average peak force of } \\
\text { shoulder musculature } \\
\text { normalized to body } \\
\text { weight pre-intervention } \\
\text { to average peak force } \\
\text { post-intervention } \\
\text { between groups. }\end{array}$ & $\begin{array}{l}\text { Two-way } \\
\text { ANOVAs with } \\
\text { one with-in } \\
\text { factor (session) } \\
\text { and one between } \\
\text { factor (group) }\end{array}$ \\
\hline 2 & $\begin{array}{l}\text { What is the effectiveness of a } \\
\text { 6-week intervention program } \\
\text { in improving scapular } \\
\text { stabilizer strength? }\end{array}$ & $\begin{array}{l}\text { Average of three trials of } \\
\text { MMT break tests measured } \\
\text { with Handheld } \\
\text { Dynamometer and then } \\
\text { normalized to body weight. }\end{array}$ & $\begin{array}{l}\text { Average peak force of } \\
\text { scapular stabilizer } \\
\text { musculature normalized } \\
\text { to body weight pre- } \\
\text { intervention to average } \\
\text { peak force post- } \\
\text { intervention between } \\
\text { groups. }\end{array}$ & $\begin{array}{l}\text { Two-way } \\
\text { ANOVAs with } \\
\text { one with-in } \\
\text { factor (session) } \\
\text { and one between } \\
\text { factor (group) }\end{array}$ \\
\hline 3 & $\begin{array}{l}\text { What is the effectiveness of a } \\
6 \text {-week intervention program } \\
\text { in improving scapular } \\
\text { kinematics during an elevation } \\
\text { task in the scapular plane? }\end{array}$ & $\begin{array}{l}\text { Kinematic data using } \\
\text { Motion Monitor, using } \\
\text { altered Euler Angles as to } \\
\text { define the local coordinate } \\
\text { systems }\end{array}$ & $\begin{array}{l}\text { Pre-intervention } \\
\text { clavicular pro/ret and } \\
\text { elev/dep and scapular } \\
\text { ant/post tipping, IR/ER, } \\
\text { and up/down tipping to } \\
\text { angles of variables post- } \\
\text { intervention between } \\
\text { groups. }\end{array}$ & $\begin{array}{l}\text { Three-way } \\
\text { ANOVAs with } \\
\text { two with-in } \\
\text { factors (session } \\
\text { and angle) and } \\
\text { one between } \\
\text { factor (group) }\end{array}$ \\
\hline 4 & $\begin{array}{l}\text { What is the effectiveness of } \\
\text { the six-week intervention } \\
\text { program in improving } \\
\text { subjects' shoulder functioning } \\
\text { and overall satisfaction? }\end{array}$ & $\begin{array}{l}\text { Self-report data from } \\
\text { SANE and DASH }\end{array}$ & $\begin{array}{l}\text { Pre-intervention score to } \\
\text { post-intervention score } \\
\text { between groups }\end{array}$ & $\begin{array}{l}\text { Two-way } \\
\text { ANOVAs with } \\
\text { one with-in } \\
\text { factor (session) } \\
\text { and one between } \\
\text { factor (group) }\end{array}$ \\
\hline
\end{tabular}




\section{CHAPTER IV}

\section{RESULTS}

\section{Subjects}

Forty-four subjects were pre-tested for participation in the current study (Table 6). Thirty-seven subjects were post tested. Three subjects were excluded due to non-compliance with the intervention program, one subject was excluded due to injury, and three subjects did not report for post-testing. The overall retention rate was $84 \%$. Subjects in the intervention group completed an average of $16.7 \pm 0.90$ sessions of the intervention program. Six additional subjects' scapular kinematic data were lost due to malfunction of the electromagnetic tracking receivers.

\section{Shoulder Strength}

The shoulder strength data are presented in Table 7 and Figures 26-31. There was a significant group by session interaction in flexion strength $\left(F_{(1,35)}=5.972, p=0.020\right)$. Post hoc pair-wise comparisons using the Bonferroni correction did not find significant differences in shoulder flexion strength pre-intervention to post-intervention in the intervention $\left(\mathrm{t}_{(20)}=-1.809, \mathrm{p}=0.086\right)$ or control group $\left(\mathrm{t}_{(16)}=1.646, \mathrm{p}=0.119\right)$. Post-hoc testing also revealed no significant difference in shoulder flexion strength between groups at the pre-intervention testing $\left(\mathrm{t}_{(35)}=-0.652, \mathrm{p}=0.518\right)$ or at the post-intervention testing $\left(\mathrm{t}_{(35)}\right.$ 
$=1.590, \mathrm{p}=0.121)$. There was no main effect of session $\left(\mathrm{F}_{(1,35)}=0.030, \mathrm{p}=0.863\right)$ or group $\left(F_{(1,35)}=0.131, p=0.720\right)$ on shoulder flexion strength.

There was also a significant group by session interaction in shoulder abduction strength $\left(\mathrm{F}_{(1,35)}=6.635, \mathrm{p}=0.014\right)$. Post hoc analysis revealed no significant differences in shoulder abduction strength pre-intervention to post-intervention in the intervention $\left(\mathrm{t}_{(19)}=\right.$ $1.189, \mathrm{p}=0.249)$ or control group $\left(\mathrm{t}_{(16)}=2.719, \mathrm{p}=0.015\right)$. Post-hoc testing also revealed no significant difference significant difference in shoulder abduction strength between groups at the pre-intervention testing $\left(\mathrm{t}_{(35)}=-1.003, \mathrm{p}=0.323\right)$ or at the post-intervention testing $\left(\mathrm{t}_{(35)}\right.$ $=1.607, \mathrm{p}=0.117)$. There was no main effect of session $\left(\mathrm{F}_{(1,35)}=0.598, \mathrm{p}=0.445\right)$ or group $\left(\mathrm{F}_{(1,35)}=0.054, \mathrm{p}=0.817\right)$ on shoulder abduction strength.

Group by session interactions were insignificant for shoulder extension $\left(\mathrm{F}_{(1,35)}=\right.$ $0.225, \mathrm{p}=0.638)$, external rotation $\left(\mathrm{F}_{(1,35)}=0.233, \mathrm{p}=0.632\right)$, internal rotation $\left(\mathrm{F}_{(1,35)}=\right.$ $2.143, \mathrm{p}=0.152)$, and adduction $\left(\mathrm{F}_{(1,35)}=0.028, \mathrm{p}=0.868\right)$ strength. However, there was a significant main effect of session on extension strength $\left(\mathrm{F}_{(1,35)}=8.783, \mathrm{p}=0.005\right)$ indicating a significant increase in extension strength after the intervention period when the data were collapsed across groups. On average, subjects' increased their shoulder extension strength by $4.16 \%$ of their body weight at post-testing. The session main effect was not significant for internal rotation $\left(\mathrm{F}_{(1,35)}=3.476, \mathrm{p}=0.071\right)$, external rotation $\left(\mathrm{F}_{(1,35)}=3.637, \mathrm{p}=0.065\right.$, and adduction $\left(\mathrm{F}_{(1,35)}=0.411, \mathrm{p}=0.526\right)$. There was no significant main effect of group in extension $\left(\mathrm{F}_{(1,35)}=0.225, \mathrm{p}=0.638\right)$, internal rotation $\left(\mathrm{F}_{(1,35)}=0.796, \mathrm{p}=0.379\right)$, external rotation $\left(\mathrm{F}_{(1,35)}=0.003, \mathrm{p}=0.959\right)$, and adduction $\left(\mathrm{F}_{(1,35)}=1.639 \mathrm{p}=0.209\right)$. 
The scapular stabilizer strength data are presented in Table 7 and Figures 32-34.

There were no significant group by session interactions in scapular retraction $\left(\mathrm{F}_{(1,35)}=0.031\right.$, $\mathrm{p}=0.861)$, scapular retraction with downward rotation $\left(\mathrm{F}_{(1,35)}=0.002, \mathrm{p}=0.967\right)$, and scapular retraction with upward rotation $\left(\mathrm{F}_{(1,35)}=0.190, \mathrm{p}=0.666\right)$ strength. However, there was a significant main effect of session on scapular retraction $\left(\mathrm{F}_{(1,35)}=55.212, \mathrm{p}<0.005\right)$ indicating a significant increase in scapular retraction strength after the intervention period when the data were collapsed across groups. On average, subjects increased their scapular retraction strength by $6.25 \%$ of their body weight at post-testing. There was no significant main effect of session on scapular retraction with downward rotation $\left(\mathrm{F}_{(1,35)}=3.668, \mathrm{p}=\right.$ $0.064)$ or scapular retraction with upward rotation $\left(\mathrm{F}_{(1,35)}=3.683, \mathrm{p}=0.063\right)$ strength. There was no significant group main effect on retraction strength $\left(\mathrm{F}_{(1,35)}=0.375, \mathrm{p}=0.544\right)$, retraction with downward rotation $\left(\mathrm{F}_{(1,35)}=0.002, \mathrm{p}=0.967\right)$, or retraction with upward rotation $\left(\mathrm{F}_{(1,35)}=0.190, \mathrm{p}=0.666\right)$

\section{Scapular Kinematics}

Scapular kinematic data are presented in Table 8. There were no significant angle by group by session three-way interactions for scapular internal/external rotation $\left(\mathrm{F}_{(4,108)}=\right.$ $0.687, \mathrm{p}=0.462)$, upward/downward rotation $\left(\mathrm{F}_{(4,108)}=0.159, \mathrm{p}=0.772\right)$, anterior/posterior tilt $\left(\mathrm{F}_{(4,108)}=0.578, \mathrm{p}=.518\right)$, protraction $/$ retraction $\left(\mathrm{F}_{(4,100)}=3.885, \mathrm{p}=.051\right)$, or elevation/depression $\left(\mathrm{F}_{(4,100)}=1.752, \mathrm{p}=0.197\right)$ (Figures 35-39). Therefore, two-way interactions (angle by group, angle by session, and group by session) and main effects (session and group) were analyzed.

There was a significant angle by group interaction on internal/external rotation $\left(\mathrm{F}_{(4,108)}=5.453, \mathrm{p}=0.018\right)$. Bonferroni post-hoc analysis demonstrated that the scapula was 
more internally rotated in the treatment group participants compared to the control group participants at humeral elevation angle of $0^{\circ}\left(\mathrm{t}_{(58)}=2.918, \mathrm{p}=.005\right)$ and $30^{\circ}\left(\mathrm{t}_{(60)}=2.840, \mathrm{p}\right.$ $=.006)$ when the data were collapsed across sessions at an adjusted alpha level of $0.01(=$ 0.05 / 5 comparisons). The scapular internal/external rotation angles were not different between groups at humeral elevation angles of $60^{\circ}\left(\mathrm{t}_{(60)}=2.327, \mathrm{p}=.023\right), 90^{\circ}\left(\mathrm{t}_{(60)}=1.271\right.$, $\mathrm{p}=.209)$, and $120^{\circ}\left(\mathrm{t}_{(60)}=0.358, \mathrm{p}=.722\right)$. The session by group $\left(\mathrm{F}_{(1,27)}=0.001, \mathrm{p}=0.973\right)$ and angle by session $\left(\mathrm{F}_{(4,108)}=1.051, \mathrm{p}=0.338\right)$ interactions were non-significant. $\mathrm{A}$ significant main effect of session were present for internal/external rotation $\left(\mathrm{F}_{(1,27)}=25.085\right.$, $\mathrm{p}<.0005)$. The swimmers' scapulae were more internally rotated at the post-intervention session compared to the pre-intervention session after averaging over the groups and angles. On average, the scapula was $11.1^{\circ} \pm 2.21^{\circ}$ more internally rotated at the post-intervention session.

There were no significant angle by group $\left(\mathrm{F}_{(4,108)}=0.993, \mathrm{p}=0.347\right)$, session by group $\left(\mathrm{F}_{(1,27)}=0.080, \mathrm{p}=0.779\right)$, or angle by session $\left(\mathrm{F}_{(4,108)}=0.894, \mathrm{p}=0.383\right)$ interactions on upward/downward rotation. The main effects for session and group were also insignificant.

There were no angle by group $\left(\mathrm{F}_{(4,108)}=0.876, \mathrm{p}=0.406\right)$, session by group $\left(\mathrm{F}_{(1,27)}=\right.$ $1.026, \mathrm{p}=0.320)$, or angle by session $\left(\mathrm{F}_{(4,108)}=1.834, \mathrm{p}=0.180\right)$ interactions for scapular anterior/posterior tilt. The main effects for session and group were also insignificant.

There was a significant angle by session interaction for protraction/retraction $\left(\mathrm{F}_{(4,100)}\right.$ $=2.560, \mathrm{p}=0.043)$. The effect of humeral elevation angle on scapular protraction/retraction angles was different between sessions after averaging over the groups. Bonferroni post-hoc 
analysis demonstrated that the scapula was more protracted after the intervention compared to before the intervention at humeral elevation angles of $0^{\circ}\left(\mathrm{t}_{(26)}=3.992, \mathrm{p}<.0005\right), 30^{\circ}\left(\mathrm{t}_{(27)}\right.$ $=3.651, \mathrm{p}=.001)$, and $60^{\circ}\left(\mathrm{t}_{(27)}=2.915 \mathrm{p}=.007\right)$ at an adjusted alpha level of $0.01(=0.05 /$ 5 comparisons). The scapular protraction/retraction angles were not different between sessions at humeral elevation angles of $90^{\circ}\left(\mathrm{t}_{(27)}=2.034, \mathrm{p}=.052\right)$ and $120^{\circ}\left(\mathrm{t}_{(60)}=1.680, \mathrm{p}\right.$ $=.104)$. There was no significant angle by group $\left(\mathrm{F}_{(4,100)}=2.560, \mathrm{p}=0.109\right)$ or session by group $\left(\mathrm{F}_{(1,25)}=1.006, \mathrm{p}=0.325\right)$ interactions. A significant main effect of session was present for protraction/retraction $\left(\mathrm{F}_{(1,25)}=10.88, \mathrm{p}=0.003\right)$. The swimmers' scapulae were more protracted at the post-intervention session compared to the pre-intervention session after averaging over the groups and angles. On average the scapula was $8.83^{\circ} \pm 2.67^{\circ}$ more protracted at the post-intervention session.

There was a significant angle by group interaction for scapular elevation/depression angles $\left(\mathrm{F}_{(4,100)}=4.320, \mathrm{p}=0.038\right)$. However, a Bonferroni post-hoc analysis did not reveal between-session differences in scapular elevation/depression angles at any humeral elevation angle. Pair-wise comparisons using Bonferroni correction were not significant at $0^{\circ}\left(\mathrm{t}_{(56)}=\right.$ $1.169, \mathrm{p}=.248), 30^{\circ}\left(\mathrm{t}_{(57)}=0.861, \mathrm{p}=.393\right), 60^{\circ}\left(\mathrm{t}_{(57)}=-0.533, \mathrm{p}=. .596\right), 90^{\circ}\left(\mathrm{t}_{(57)}=-0.971\right.$ $\mathrm{p}=.335)$, or $120^{\circ}\left(\mathrm{t}_{(57)}=-1.722, \mathrm{p}=.091\right)$. The session by group $\left(\mathrm{F}_{(1,25)}=3.875, \mathrm{p}=0.060\right)$ and angle by session $\left(\mathrm{F}_{(4,100)}=0.671, \mathrm{p}=0.438\right)$ interactions were not significant. A significant main effect of session was present for scapular elevation/depression $\left(\mathrm{F}_{(1,25)}=\right.$ $4.279, \mathrm{p}=.049)$. The swimmers' scapula was more elevated at the post-intervention session compared to the pre-intervention session after averaging over the groups and angles. On average, the scapula was $2.85^{\circ} \pm 1.38^{\circ}$ more elevated at the post-intervention session. 


\section{SANE and DASH}

The SANE and DASH subjective ranking data are presented in Table 9 and Figure

40-41. There was no significant group by session interaction for SANE composite score $\left(\mathrm{F}_{(1,35)}=0.011, \mathrm{p}=.916\right)$. There was no significant group by session interaction for DASH composite score $\left(\mathrm{F}_{(1,35)}=2.047, \mathrm{p}=.160\right)$. There was no main effect of session on SANE $\left(\mathrm{F}_{(1,35)}=0.024, \mathrm{p}=.877\right)$ or DASH composite score $\left(\mathrm{F}_{(1,35)}=0.258, \mathrm{p}=.615\right)$ after the data were collapsed across groups. There was no significant main effect of group on $\operatorname{SANE}\left(\mathrm{F}_{(1,35)}\right.$ $=0.452, \mathrm{p}=0.505)$ or DASH composite score $\left(\mathrm{F}_{(1,35)}=0.668, \mathrm{p}=0.419\right)$. 


\section{CHAPTER V}

\section{DISCUSSION}

The purpose of this study was to determine the effects of a 6-week intervention program on shoulder strength and scapular kinematics in division I collegiate swimmers. Validating a shoulder-strengthening and stretching program for collegiate swimmers that modifies the suggested risk factors of shoulder pain provides clinicians, coaches, and athletes with a specific program to incorporate into their training and potentially reduce the incidence of shoulder pain during a competitive season.

\section{Shoulder and Scapular Stabilizer Strength}

While the intervention program did not result in statistically significant improvements in shoulder strength variables, there were non-significant trends indicating intervention group subjects may have stronger flexion and abduction strength compared to the subjects in the control group after the training. These trends resulted from a modest strength gain in the intervention group and a small strength loss in the control group. Subjects in the intervention group gained an average of $2.0 \%$ of their body mass in shoulder flexion strength, while subjects in the control group lost an average of $2.3 \%$. Similarly, subjects in the control group gained an average of $1.7 \%$ of their body weight in shoulder abduction strength, while subjects in the control group lost an average of $3.1 \%$. The strength gain observed in the intervention group was small and may not be clinically significant. However, the trends 
indicate that continuation of the exercise program over a longer period of time may result in clinically significant strength gain. The shoulder flexion, internal rotation at $90^{\circ}$, and external rotation at $90^{\circ}$ exercises included in the intervention program has been shown to successfully activate the deltoids (Myers, Pasquale et al. 2005). These exercises may have contributed to the increase in shoulder flexion and abduction strengths.

A previous study examining the adduction to abduction strength ratio in competitive swimmers has reported that the swimmers had greater adduction to abduction strength ratio of 2.05 compared to 1.53 in non-swimmers (McMaster 1994; Pink and Tibone 2000). The authors attributed this unique strength ratio observed in swimmers to sports specific adaptations due to the demands for powerful adduction and internal rotation movements to propel the swimmer through the water. The adduction to abduction strength ratio in swimmers who participated in this study were $1.35-1.37$ at the time of the pre test, which is much lower than the swimmers or the non-swimmers that participated in the study by McMaster et al. Swimmers who participated in this study were elite collegiate swimmers who has been performing strength training and dry land training under the direction of strength and conditioning specialists. Participation in organized weight training program may have resulted in more balanced strength ratio. Also, the original ratio was taken at the beginning of a training season, and therefore the strength ratio profile normally present in swimmers may have not been evident at the time since adduction to abduction strength ratio has been reported to increase over the course of the season (Beach, Whitney et al. 1992). Shoulder adduction and internal rotation occurring from forceful contraction of the pectoralis major and latissimus dorsi are responsible for propulsion in swimming. Greater adduction to abduction strength ratios may be caused by overdevelopment of the pectoralis major and 
latissimus dorsi due to the demands of the sport (Pink, Perry et al. 1991). Overdevelopment of these muscles has been linked to shoulder injuries because the muscles can pull the clavicle and scapula forward creating forward shoulder posture. This posture is theorized to alter the length-tension relationship of the shoulder external rotators and abductors muscle to decrease the force produced by the muscle (Pink and Tibone 2000; Kendall, McCreary et al. 2005) as well as to alter scapular and glenohumeral kinematics to decrease subacromial space, which makes individuals more vulnerable to shoulder injury (Culham and Peat 1993; Solem-Bertoft, Thuomas et al. 1993; Ludewig, Cook et al. 1996; Kebaetse, McClure et al. 1999; Michener, McClure et al. 2003). Therefore, developing a more balanced strength profile, by having an optimal adduction to abduction ratio, may decrease the susceptibility of swimmers to overuse shoulder injuries by improving posture and scapular positioning. While statistically insignificant, the study demonstrated a trend that the strength ratio in the control group increased from 1.37 to 1.60 , while the ratio in the intervention group decreased from 1.35 to 1.29 . This trend may indicate that the intervention program may have had an effect in negating the change in strength ratio occurring with swim training, and therefore could have a protective effect on the development of shoulder injuries.

The program did not result in improvements of the shoulder adduction, extension, internal rotation and external rotation strength or the scapular stabilizer strengths (scapular retraction, retraction and downward rotation, and retraction and upward rotation), thus our hypotheses that the program would improve shoulder and scapular muscles were rejected. Shoulder adduction, extension, and internal rotation are the primary movements required to propel the body through the water during swimming and the loads required during swimming most likely are stronger than what can be applied using resistive tubing. External rotation is 
the primary motion during the recovery phase of swimming, thus the muscles that produce this motion are activated on every stroke of swimming (Yanai, Hay et al. 2000). With the amount of yardage that the swimmers were completing and the concurrent demands on these muscle groups, the resistive tubing most likely did not provide enough resistance to create strength improvements.

To date, only a few studies have investigated the effectiveness of the intervention program to improve shoulder strength specifically in swimmers. Results from this study were similar to those from a study in collegiate swimmers by Swanik et al (Swanik, Swanik et al. 2002) that found no significant strength differences between control and intervention groups after a 6-week intervention program using elastic tubing. Like the current study, this intervention program was conducted during preseason training. Swanik et al (Swanik, Swanik et al. 2002) attribute the lack of significant findings to the fact that large increases in strength occur during the preseason. Thus, the increase in swimming elicited strength gains that overshadowed any benefits that may have been caused by the strengthening program that was used in Swanik's study, suggesting that the intervention program may be more effective if implemented at a different point in the season.

Effectiveness of intervention programs in improving shoulder strength has been evaluated in patients with shoulder injuries. A study by Ramsi et al (Ramsi, Swanik et al. 2004) evaluated the change in shoulder internal and external rotator strength over the course of the competitive season in high school swimmers, and found that the swimmer's internal and external rotation strength improved during the season. While their results were not consistent with the results found in this study, the participants in the study by Ramsi et al were high school swimmers who did not perform group weight training and had lower 
physical training demands as collegiate swimmers. Furthermore, while the internal and external rotation strength improvements were not observed in the current study, it is possible that overtraining may have resulted in decrease in muscle strength, negating any improvements gained from the intervention program. During this portion of the season, the focus is on building endurance and every swimmer is swimming a significant number of yards, approximately 7,000 yards per practice with 9 practices a week. In addition to practice, swimmers are completing weights and dry land training 5 days per week. This extreme amount of training causes fatigue in the swimmers, which may be observed in the posttesting. A significant decrease in shoulder external and internal rotation strength, of up to $20 \%$ of baseline strength, has been found in individuals following a fatigue protocol (Tyler, Cuoco et al. 2009). Although this study evaluated muscular strength immediately following the fatigue protocol, no studies observing the effects of overtraining throughout a competitive season have been conducted. The results of the current study may be more indicative of fatigue and overtraining, as an increase in strengths due to swim conditioning, dry land workouts, and weight sessions would be expected. However, currently, no studies have observed the effects of overtraining on shoulder and scapular stabilizer strength throughout a competitive season.

Wang et al (Wang, McClure et al. 1999) introduced a 6-week intervention program involving shoulder strengthening and stretching in subjects with impingement. After the intervention, a significant increase in isometric strength for external rotation, internal rotation and horizontal abduction occurred. McClure et al (McClure, Bialker et al. 2004) also evaluated strength changes following an intervention program in subjects with impingement, and reported increased strength in abduction and internal rotation. The current study did not 
find a significant improvement in shoulder of scapular muscle strengths. Results of the current study may differ from the intervention program by Wang and McClure because of the differences in subject population. Subjects in their studies were experiencing impingement symptoms at the time of testing and were not competitive athletes. A study by Bak and Magnusson (Bak and Magnusson 1997) found that swimmers with impingement symptoms have decreased internal rotation strength compared to the healthy subjects as a result of their pain. The impingement patients likely had weaknesses related to their injury and pain at the baseline testing, which recovered with resolution of the symptoms. The healthy collegiate swimmers participated in the current study were already strong and therefore may take greater stimuli to produce strength gains. The principle of overload states that our bodies adapt to a workload and will not make improvements unless the workload is increased (Cissik 2002).

While the program did not result in statistically significant improvements in strength, the results suggest that shoulder extension and scapular retraction strength both increased from pre-testing to post-testing regardless of whether the athletes performed the intervention program. These strength increases are most likely due to a pull-up and push-up program that the team completed at the beginning of the season. Pull-ups are aimed at increasing strength in the rhomboids, middle and lower portions of the trapezius and latissimus dorsi and pushups increase strength in the triceps, pectoralis major, and anterior deltoid and are done to increase the propulsive force during swimming, especially during the pull phase of swimming (McLeod 2010). The increase in strength in the rhomboids, upper and middle trapezius, latissimus dorsi, and triceps would explain the increase in shoulder extension and retraction strength. In addition, the pre-tests were performed before any team training began, 
and therefore combination of dry land workouts, weight training, and swim conditioning likely resulted in strength gains and introduction of elastic tubing did not result in additional strength gain.

\section{Scapular Kinematics}

This study evaluated scapular kinematics between intervention and control subjects before and after an intervention period. The hypotheses of the current study were that the intervention exercise would result in increased scapular upward rotation, posterior tipping, external rotation, retraction, and elevation in the intervention group compared to the control group. These hypotheses were not supported by the current study since the results indicate that the exercise program had no effect on scapular kinematic variables at any humeral elevation angle. The subjects in the intervention group had significantly greater scapular internal rotation compared to the control group at $0^{\circ}$ and $30^{\circ}$ of humeral elevation. Since the subjects were randomly assigned to the groups, it is unknown why the difference was found. However, since the shoulder pain in overhead athletes is commonly experienced at higher humeral elevation angle, this difference is likely not related to swimmer's vulnerability to shoulder impingement. Myers et al (Myers, Hwang et al. 2009) found that individuals with impingement had poor force coupling of the subscapularis-infraspinatus and supraspinatusinfraspinatus at humeral elevation angles above $90^{\circ}$. The authors suggest that these altered patterns of muscular co-activation lead to a decreased ability to oppose anterior humeral head migration further contributing to decreased subacromial space in impingement patients. The altered patterns of rotator cuff activation was not found at lower angles of humeral elevation, thus the scapular kinematic alterations found in the current study are not related to muscular activation patterns that promote impingement. 
Interestingly, this study found that swimmers' scapulae became more internally rotated, protracted, and elevated at the post-intervention screening compared to the preintervention screening regardless of the group assignment. At the pre-screening, the swimmers in the study were just returning from summer break and had not been training intensely. The changes in scapular kinematics may be attributed to increased tightness of the posterior shoulder and pectoralis major and minor muscles that developed in response to increasing training intensity. Borich et al (Borich, Bright et al. 2006) found that subjects with posterior shoulder tightness, which commonly develops in overhead athletes, causes demonstrated abnormal scapular positioning, specifically increased anterior tilt and decreased upward rotation. Borstad and Ludewig (Borstad and Ludewig 2005) found that a tight pectoralis minor is associated with increased scapular anterior tipping and internal rotation. Therefore, muscle imbalances and tightness in the pectoralis major and minor muscles and posterior shoulder structures that develops due to the increased swim training may be responsible for the increased protraction and internal rotation at the post testing.

The findings from this study are consistent with the study by McClure at el (McClure, Bialker et al. 2004) in that the intervention program did not have any effect on scapular kinematics. On the contrary, a study by Wang et al (Wang, McClure et al. 1999) observed a decreased upward rotation and elevation and an increased internal rotation following an exercise program in asymptomatic participants with forward shoulder posture. However, the change in scapular kinematics may have been attributed to the fact that the all participants in the study had forward head posture associated with tightness of the pectoralis major and minor muscles. Therefore, stretching of the tight musculature may have resulted in change in scapular kinematics. While forward shoulder posture and muscle tightness of the pectoralis 
major and minor muscles are common among swimmers, not all swimmers have this posture or muscular tightness, especially at the beginning of the season (Johnson 1988; Bak and Fauno 1997).

The increases in scapular internal rotation, protraction, and elevation that were found after the intervention period may put the swimmer at an increased risk for shoulder impingement because of increased contact of the acromion and rotator cuff tendons from decreased subacromial space (Ludewig and Cook 2000; Michener, McClure et al. 2003; Ludewig and Reynolds 2009). Ludewig and Cook demonstrated that subjects with symptoms of impingement syndrome have increased internal rotation. Internal rotation of the scapula during a humeral elevation task serves to move the acromion more anteriorly, increasing the contact with the rotator cuff tendons (Ludewig and Reynolds 2009). Solem-Bertoft et al (Solem-Bertoft, Thuomas et al. 1993) found that the subacromial space narrowed as the shoulder moved from a retracted position to a protracted position. Narrowing of the subacromial space results in increased contact between the humerus and the underlying structures, which increases the likelihood of developing shoulder impingement (Fu, Harner et al. 1991; Solem-Bertoft, Thuomas et al. 1993). In addition to the adaptive change in response to the stress imposed on the shoulder, muscular fatigue from overtraining may be attributed to the alteration in scapular kinematics. A previous study has demonstrated that fatigue of the external rotator muscle resulted in increased scapular internal rotation, posterior tipping, and upward rotation primarily at the lower ranges of motion (Tsai, McClure et al. 2003). Increased scapular elevation could be due to a tight upper trapezius muscle. The upper trapezius muscle serves an important role of stabilizing the scapula on the thorax during dynamic movement, but often becomes overdeveloped to its tendency to compensate for 
weakness in other stabilizing muscles, especially as individuals begin to experience impingement symptoms (Wadsworth and Bullock-Saxton 1997; Cools, Witvrouw et al. 2003; Moraes, Faria et al. 2008). Previous studies have shown that the upper trapezius muscle functions to elevate scapula (Kibler 1998; Cools, Witvrouw et al. 2003; Moraes, Faria et al. 2008). Therefore, while no study to date has demonstrated the relationship between upper trapezius tightness and altered scapular kinematics, it can be hypothesized that a tight upper trapezius muscle would lead to increased scapular elevation angles.

Overall, subjects in the study had an increase in scapular internal rotation, protraction, and elevation, regardless of group assignment likely due to the muscle tightness that developed in response to intense swimming training. The intervention program was unable to reverse this effect.

Scapular kinematic changes observed in this study are related to shoulder injuries, so it is important that the intervention program addresses these changes. Additional stretching exercise that focuses on stretching the posterior capsule, anterior musculature, and upper trapezius may be necessary to address these changes. McClure et al (McClure, Balaicuis et al. 2007) reported that the cross-body stretch is effective at stretching the posterior capsule and has slightly greater improvements in internal rotation deficits than the sleeper stretch. Since just the sleeper stretch was used as a part of the intervention program in the current study, the cross-body stretch could be added to provide additional stretching to the posterior capsule. In addition, anecdotal evidence has found increased benefits of the sleeper stretch if it is performed using a "contract-relax" technique, which could be incorporated into the intervention program. An additional stretch for the pectoralis major and minor could be added, as the corner stretch included in this study did not create enough improvements to 
influence the scapular kinematics. A potential stretching exercise to include in future studies is performed by having the individual lay over a foam roller with a partner pushing their shoulders down. This stretch isolates the pectoralis muscles in a safe position, without causing anterior displacement of the humeral head, which promotes anterior instability of the shoulder (Weldon and Richardson 2001; Johnson, Gauvin et al. 2003; Tovin 2006). Finally, stretching for the upper trapezius muscle may be added to counteract the clavicular elevation that was found.

\section{SANE and DASH score}

The intervention program did not result in a significant improvement in the subjective measures of shoulder satisfaction and functioning thus these hypotheses were rejected. The SANE score has been shown to be a reliable and valid measure of shoulder functioning in the general population, but has not been used in the athletic population as a measure of shoulder functioning (Lysholm and Gillquist 1982; Tegner and Lysholm 1985; Williams, Taylor et al. 2000). The SANE asks subjects how their shoulder felt at a specific time and the score may only reflect how the individual's shoulder felt at the time of testing, and not the individual's general perception of their shoulder function. A one time assessment of shoulder functioning may not be valid for an athletic population, as perception of shoulder pain and functioning may be influenced by weight lifting or training performed on the particular day and also influenced by the time of the competitive season. Subjects were pretested at the beginning of the year when all training was focused on skill development and the practice was held only 3 times per week. Post testing was conducted in a midst of in-season training when the swimmers performed high volume practice and high intensity weight lifting program, and therefore comparison of the reporting before and after the intervention period may be invalid. 
In addition, subjects in the intervention group often reported being sore following the training session. Subjects were post-tested 2 days after completing a training session and this may have impacted the score that they reported.

While the SANE asks how the subject is feeling at a specific time, the DASH asks about symptoms and functioning in the past week, which may be a better indicator of the individual's general perception on the condition of their shoulder. The current study did not demonstrate statistically significant change in DASH score from the intervention program. However, interestingly there was a trend towards participants in the intervention group having higher DASH score indicating more difficulty performing sport activities, compared to the participants in the control group at the post test. This trend is contrary to the finding from a study by Swanik et al (Swanik, Swanik et al. 2002) that found a decreased incidence of reported shoulder pain in collegiate swimmers after completing a 6-week intervention program to address strength and range of motion deficits. The measure of functioning in the Swanik study was based on the reporting of interfering pain to the certified athletic trainer. If pain interfered with swimming practice and presented as dull aching pain at night, while swimming, or a feeling of the shoulder being tired, this was reported as an incidence of pain. This definition of pain may be too broad to truly capture shoulder functioning in swimmers, since shoulder pain is commonplace in the sport and swimmers are taught that pain is a component of the sport. Similar to the results from the study by Swanik et al, McClure et al (McClure, Bialker et al. 2004) reported improvement in University of Pennsylvania shoulder scale score following a 6-week intervention in subjects with impingement syndrome. However, the subjects in McClure's study had painful shoulder at the beginning of the study and the subjective scale was used to rate symptoms while receiving treatment. The subjects 
who were completing the intervention program often reported being sore after completing the strengthening program. The soreness from muscle development in the intervention program may have caused the subjects to report increased symptoms. Although it is not ideal to have increased symptoms, this soreness may be necessary to achieve strength gain.

\section{Clinical Significance}

The intervention program used in this study was designed to address weakness, muscular imbalances, and tightness commonly present in swimmers' shoulders. The weakness, muscle imbalance, and tightness targeted in this study are also considered potential contributors to shoulder injuries. The intervention program resulted in nonsignificant trends towards improvement in shoulder flexion, abduction, and adduction to abduction ratio, but did not result in significant improvement in other strength variables (extension, adduction, internal / external rotation, scapular retraction, retraction with downward rotation, and retraction with upward rotation), scapular kinematics, or subjective perception of shoulder function. Based on the findings of the current study, the intervention program was not successful in improving the variables as hypothesized and would not be an effective program to implement in competitive swimmers. However, the results of the current study did provide valuable framework for how the intervention program could be modified to benefit the competitive swimmer.

Despite the shoulder intervention program, all subjects moved into increased scapular internal rotation, protraction, and elevation, likely caused by increased tightness of the pectoralis major, pectoralis minor, and upper trapezius muscles. The exercises and stretching in this program were not strong enough to counteract the effects of swim training on muscle tightness development. A greater focus on stretching of the anterior shoulder musculature 
and posterior capsule to reverse the increasing scapular internal rotation and protraction, as well as the upper trapezius to reverse the increasing scapular elevation may be needed.

It was found that shoulder extension and scapular retraction significantly increased between sessions, regardless of group assignment. This is likely due to a push-up and pull-up program that the entire team performed as a part of the dry land program or overlap with exercises that are performed in the weight room. Therefore, exercises that targeted shoulder extension and scapular retraction strength (ex. shoulder extension and scapular retraction (T) exercises) could be eliminated from the program to shorten the exercise program, which may improve compliance to the exercise program. Additionally, internal rotation exercise may be removed from the program, as internal rotation is the movement primary responsible for propulsion during swimming and performance of exercise is unlikely to produce additional strength gain. Finally, coordination with the strength and conditioning staff is needed, to ensure that there is not an overlap between exercises, which would decrease compliance to the program and cause excessive fatigue. The intervention program should be adjusted based on the weight room requirements for the specific team.

Finally, the timing and length of the program may be important when introducing an intervention program. Fatigue, muscle soreness, and overtraining are all very common in swimmers during the pre-season training. Any positive effects of the intervention program may have been overshadowed by the physiological changes that occur due to the intense swim training. The intervention program may be able to produce more robust effect if implemented during spring training, when the focus is more on technique than yardage. The intervention program in the current study was performed for only 6 weeks, while swimmers train over 40 weeks per year. Continuing the intervention program throughout the season 
may result in greater improvements in strength, as well as long- term effects of establishing normal strength ratios, scapular kinematics, and movement patterns to prevent injury.

\section{Limitations}

There were limitations in the current study. Swimmers have been taught that shoulder pain is normal in the sport, and shoulder pain is often unreported until it is debilitating. It is possible that subjects who were experiencing shoulder pain throughout the intervention period or at post-test were included in the study due to lack of reporting. Previous studies have found that individual with shoulder pain will exhibit shoulder strength weakness (Scovazzo, Browne et al. 1991; Pink and Tibone 2000; Tyler, Cuoco et al. 2009) and altered scapular kinematics (Lukasiewicz, McClure et al. 1999; Ludewig and Cook 2000; Endo, Ikata et al. 2001; Ludewig and Reynolds 2009), therefore inclusion of the patients with unreported pain may have influenced the results. Another limitation of this study was that individual effort could not be assessed. Although the exercise program was explained to participants and a bi-weekly evaluation of technique was performed, some participants may have chosen resistive rubber tubing that was too easy.

Data collection and implementation of the strengthening program occurred immediately following a break from swimming, which is the time period when swimmers are building their cardiovascular endurance by swimming a significant number of yards at a high intensity, which may have affected the results. Overtraining and the intensity of the swim conditioning may have masked the effects of the intervention program. Strength, scapular kinematics and subjective perception of shoulder function may have been affected by muscular fatigue and muscular adaptations from the high intensity swim training. However, the study was conducted during the pre-season training to ensure control of subjects, as 
tapering, breaks from swimming, different training between individuals depending on their summer goals, and individual weight programs that begin later in the season.

\section{Future Research}

As discussed, swimming places a tremendous amount of stress on the shoulders of the athletes. The physical characteristics and sport specific demands on swimmers are different than any other sport and therefore sports specific dry land exercise program that can supplement or incorporated into the weight lifting regimen designed by the strength and conditioning coaches may be needed. More research is needed to develop an evidenced based training program and guidelines for effective and safe exercises. Implementation of the evidence based exercise program tailored to the sport specific need may decrease the stress on the shoulder and may result in prevention of the development of shoulder pain.

Additionally, a long term prospective study assessing the effectiveness of the intervention program in reducing the risk of shoulder injury is needed to truly determine how effective a strengthening program is in preventing a shoulder injury.

Finally, research examining shoulder injuries and prevention programs in swimmers of all ages is necessary. Club swim teams begin with 5 year olds and many swimmers are swimming 2 practices a day by the time they are 14 years old. These young swimmers rarely participate in weight lifting or dry land exercises organized by the team. Implementing an intervention program in youth swimming may have a larger impact on these developing muscles and prevent shoulder pain and injuries. In addition, implementing a program in younger individuals that is performed throughout their swimming career may promote physical characteristics that prevent shoulder injuries from developing later in their careers. A similar study design as the current study could be implemented in a club swimming team. 


\section{Conclusions}

The results of the current study did not find significant changes in shoulder or scapular stabilizer strength, scapular kinematics, or subjective shoulder rankings between groups. In addition, it was found that all subjects moved into increased scapular internal rotation, protraction, and elevation due to the demands of increased swim conditioning. Further research is needed to develop intervention programs in swimmers and its long-term effect on injury prevention. 
Figures

Figure 1: Shoulder Flexion

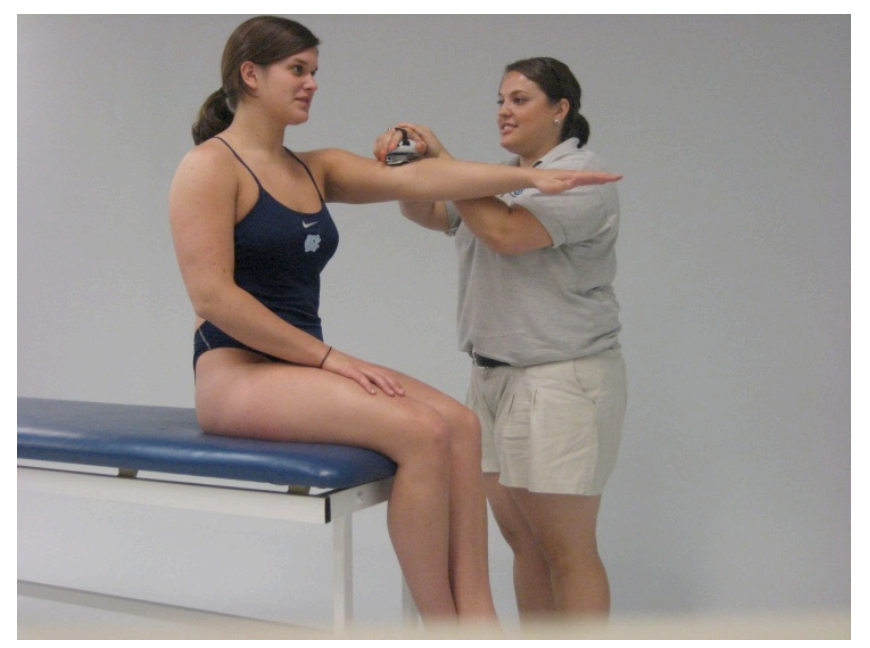

Figure 2: Shoulder Extension

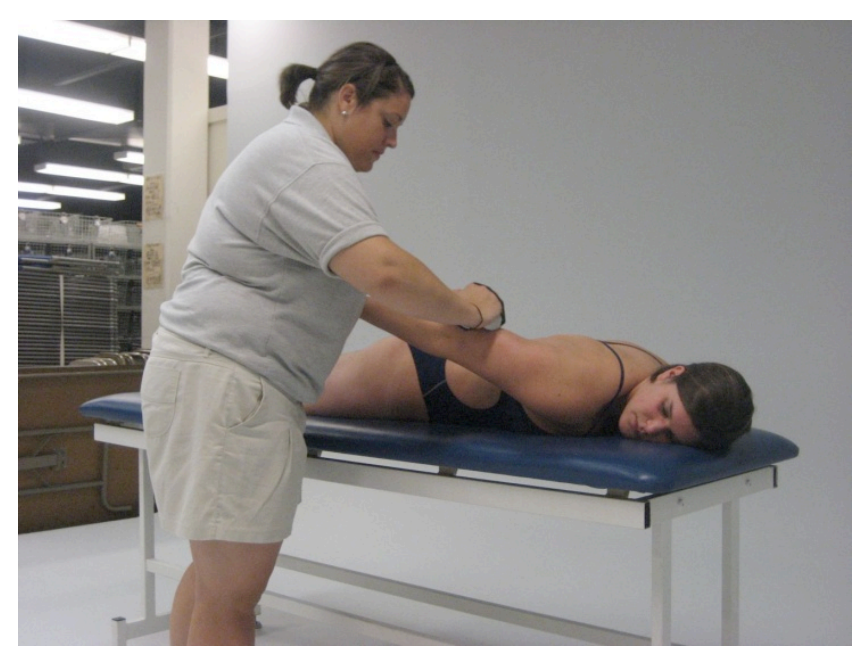


Figure 3: Shoulder External Rotation

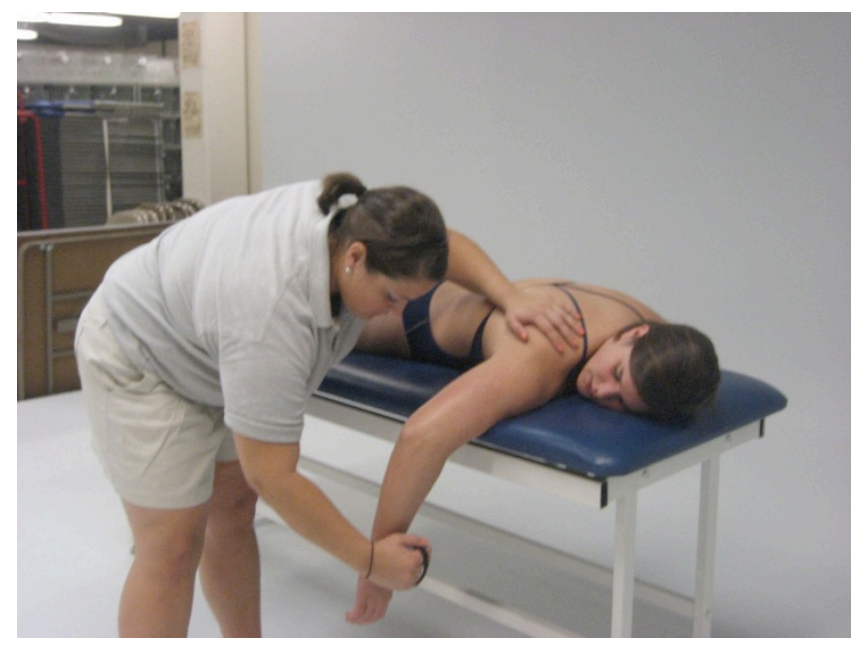

Figure 4: Shoulder Internal Rotation

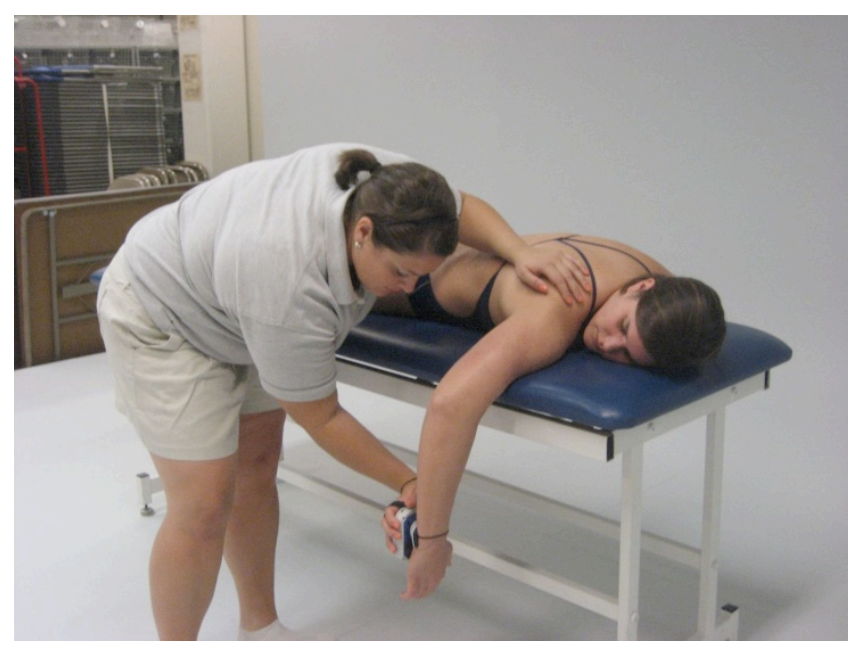

Figure 5: Shoulder Abduction

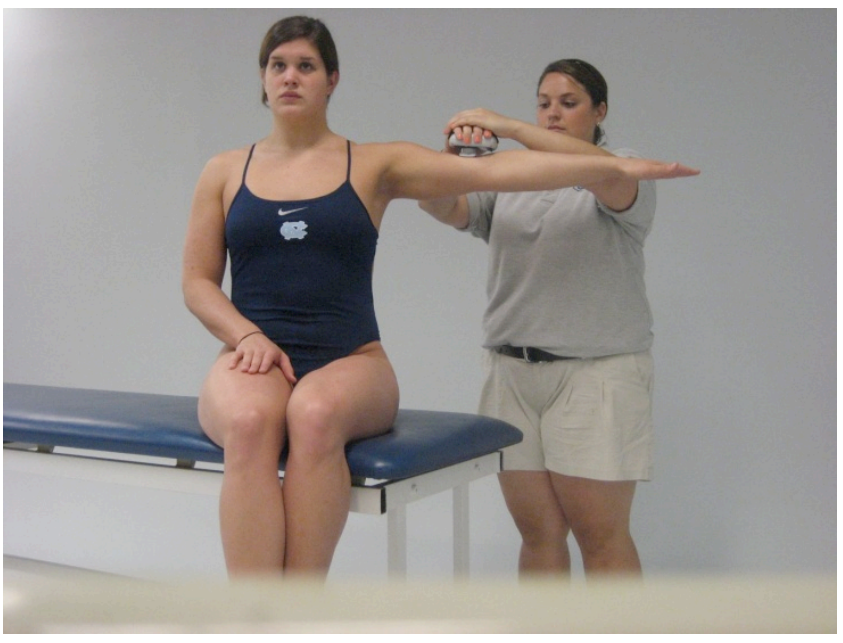


Figure 6: Shoulder Adduction

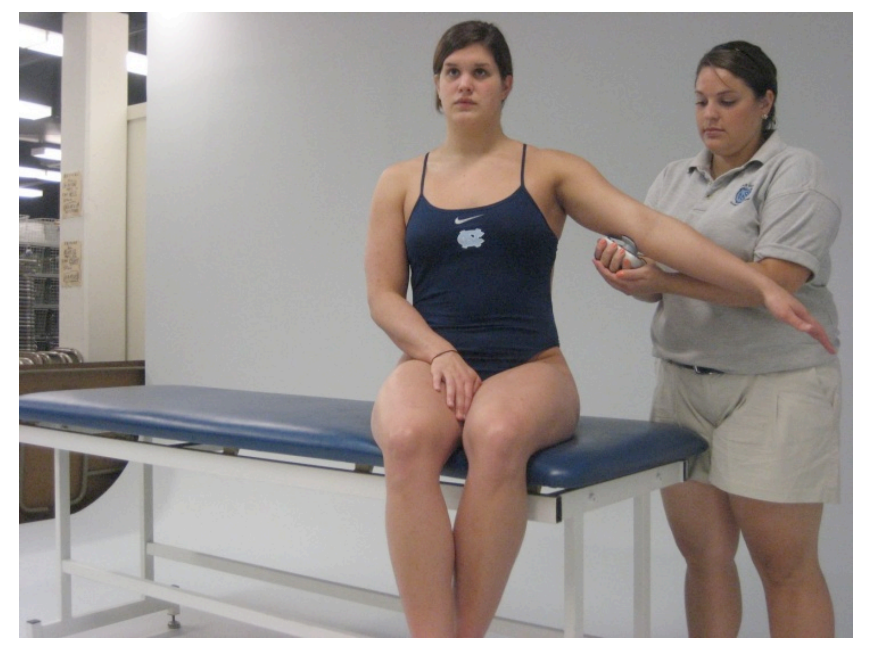

Figure 7: Scapular Retraction

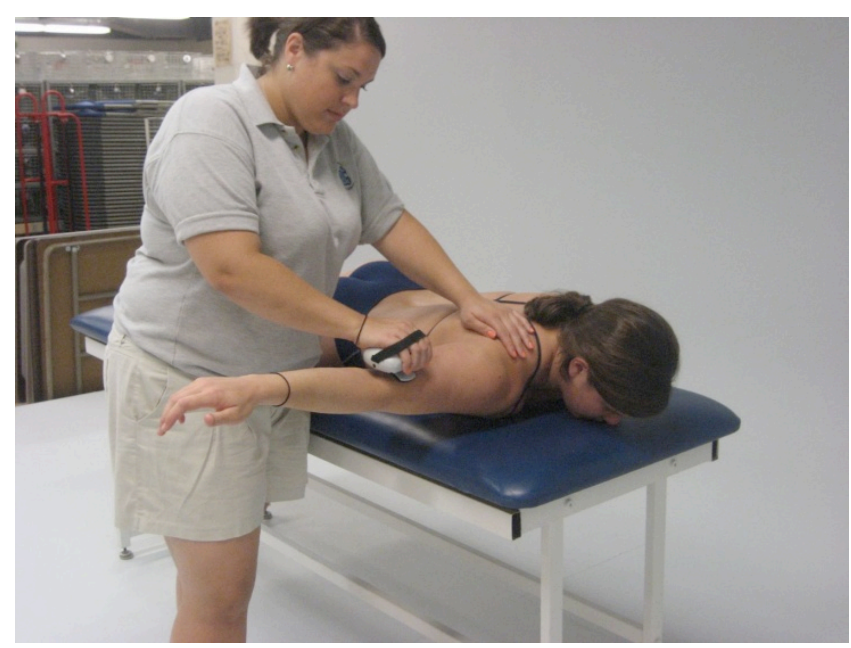

Figure 8: Scapular Retraction with Upward Rotation

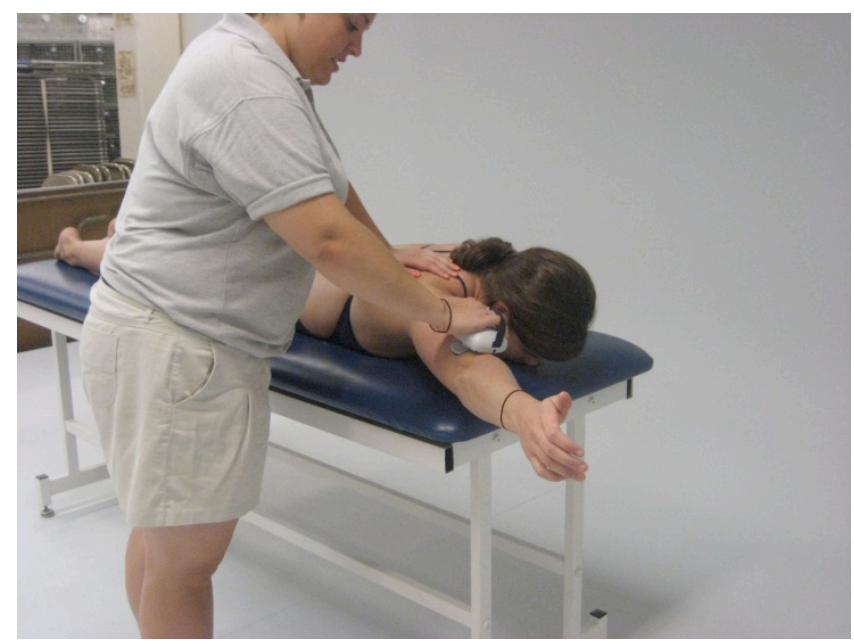


Figure 9: Scapular Retraction with Downward Rotation

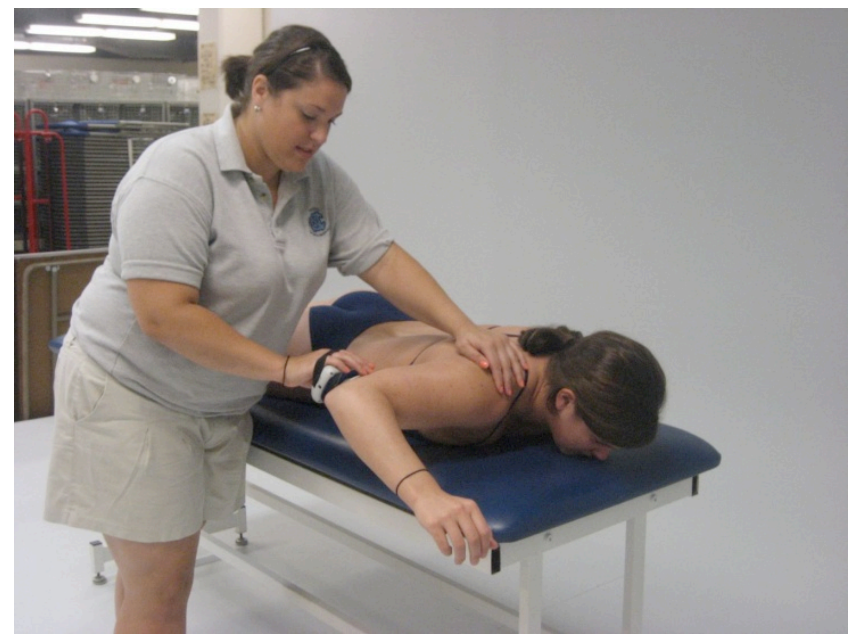

Figure 10: Assessment of Scapular Kinematics

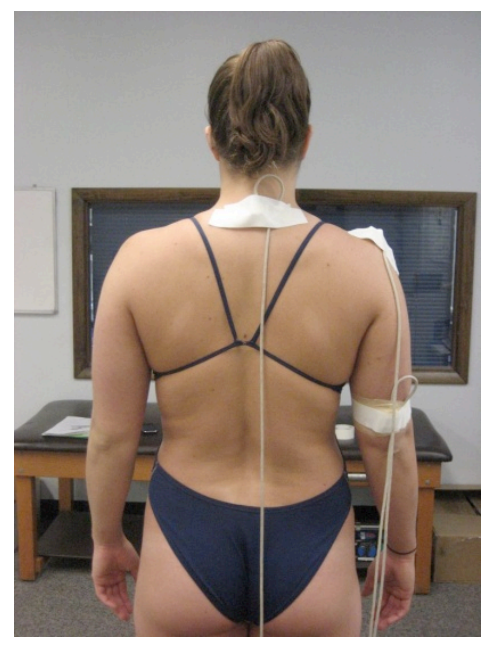

Figure 11: Shoulder Flexion
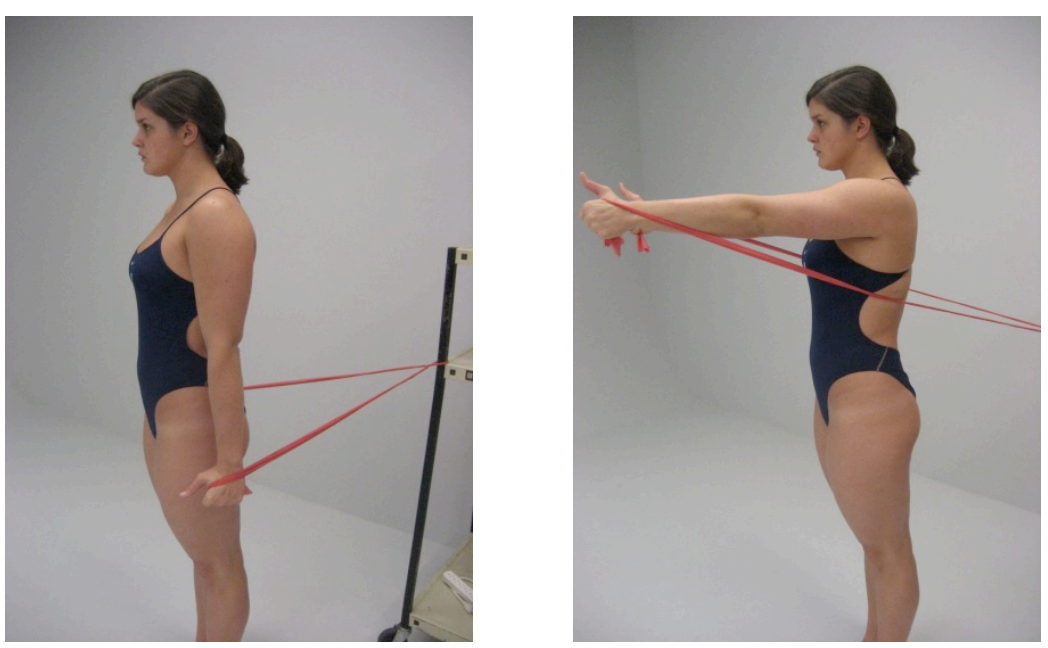
Figure 12: Shoulder Extension
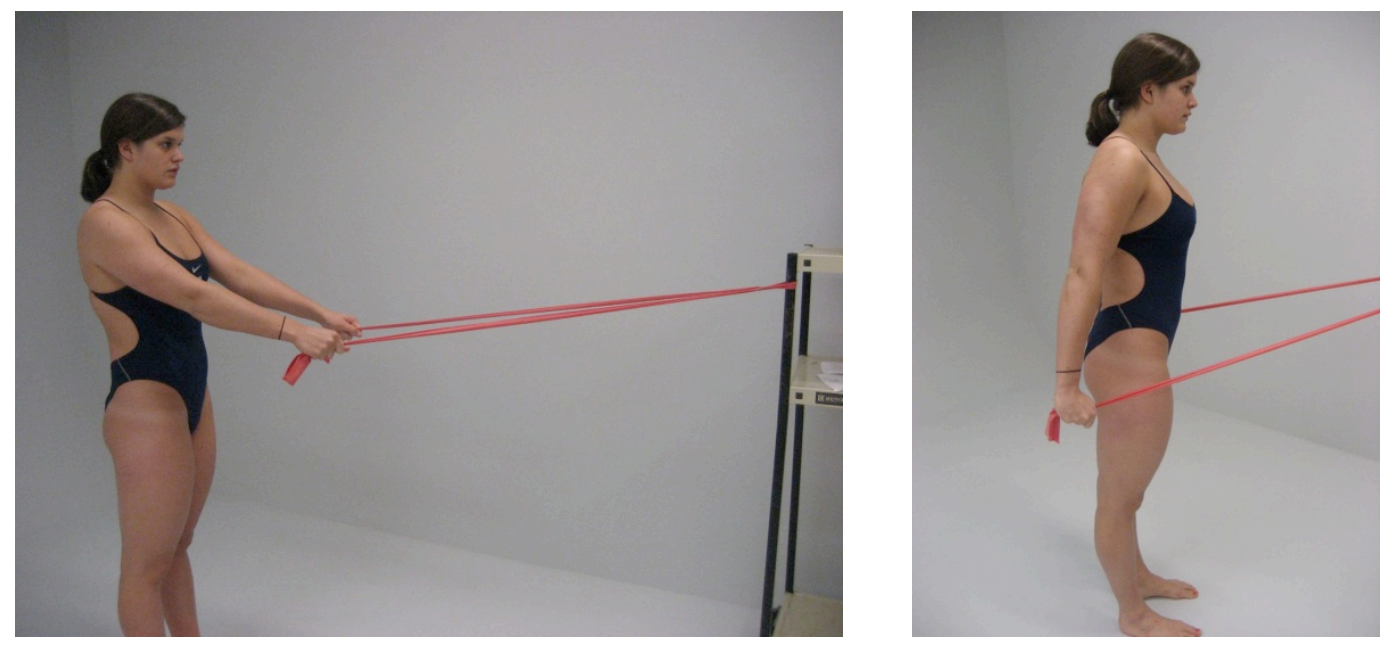

Figure 13: Shoulder ER at $90^{\circ}$
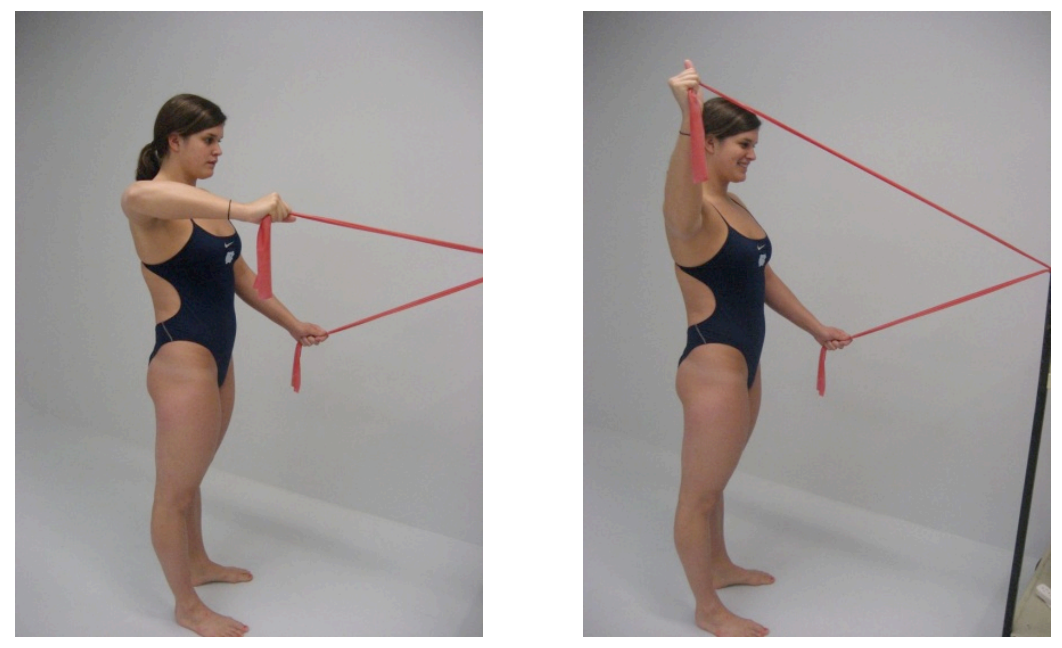

Figure 14: Shoulder IR at $90^{\circ}$
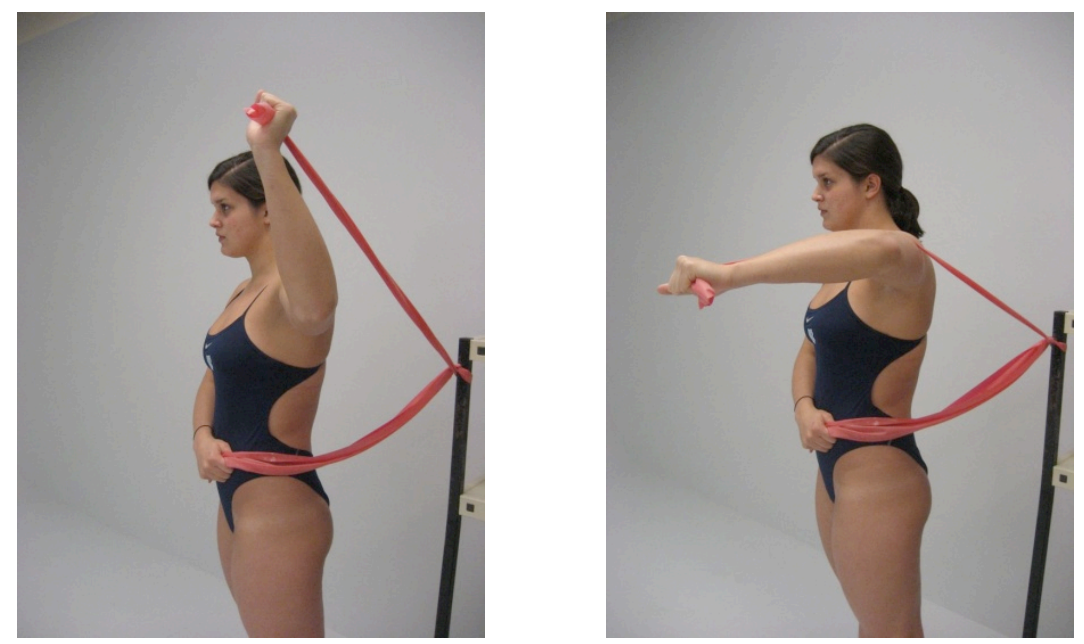
Figure 15: Low Rows
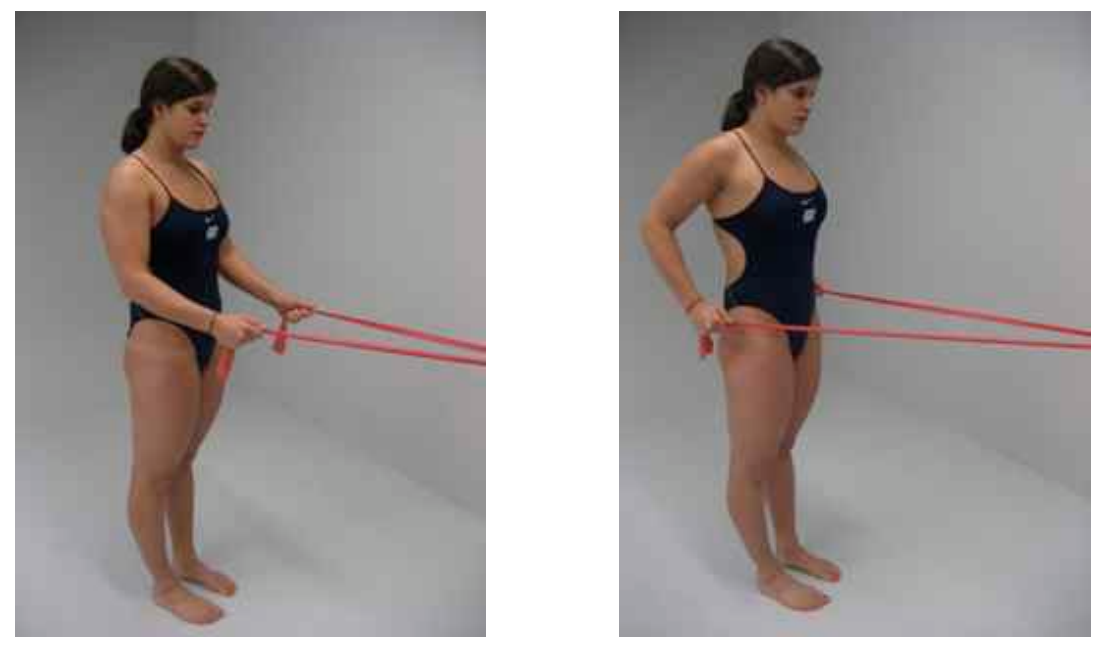

Figure 16: Throwing Acceleration
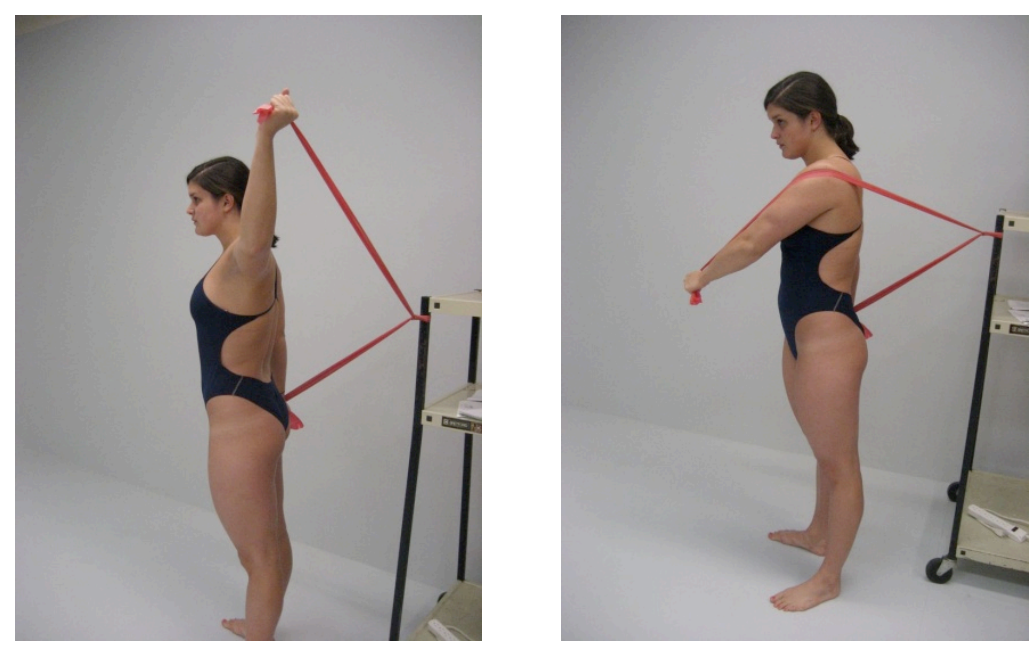
Figure 17: Throwing Deceleration

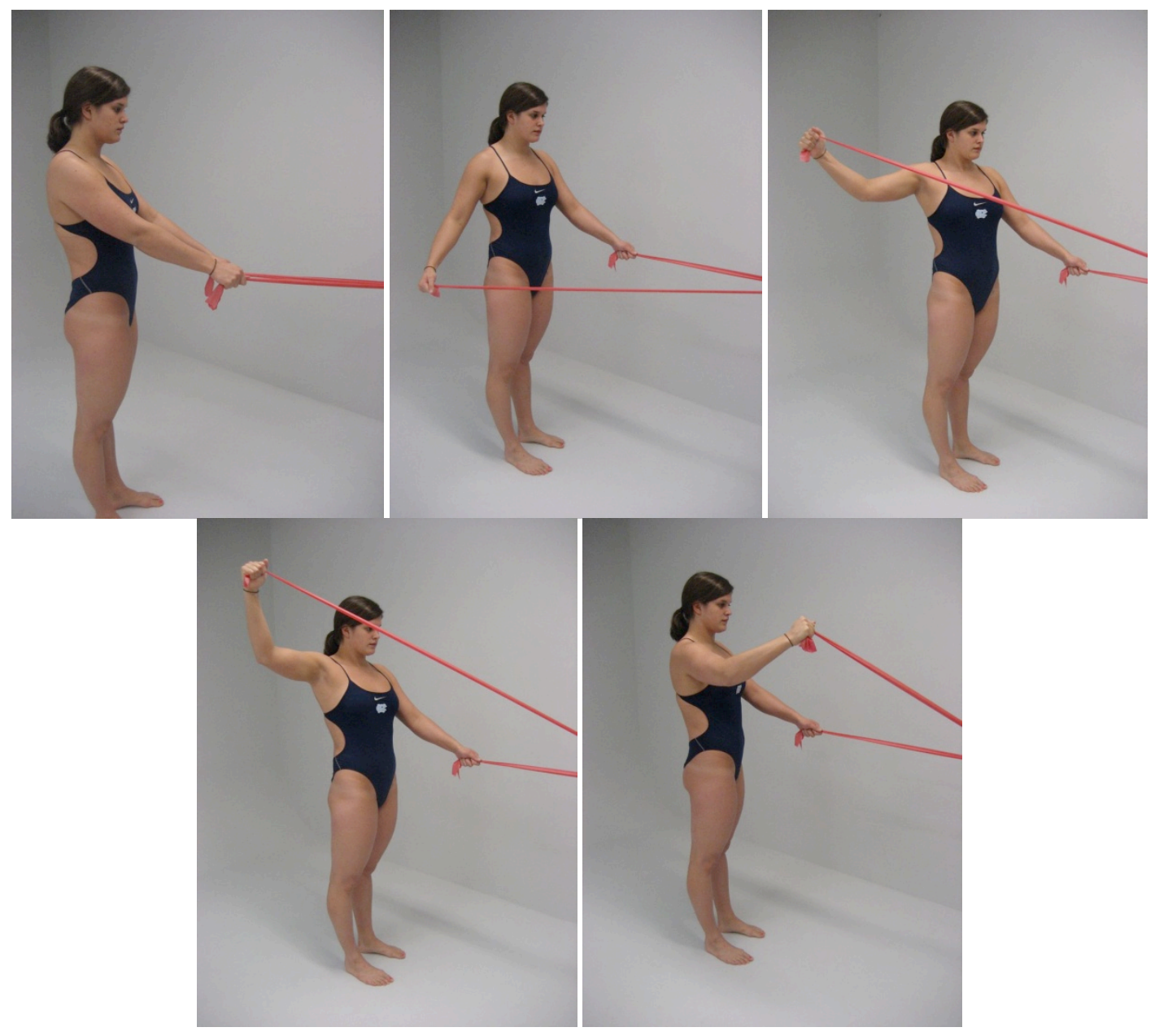

Figure 18: Ys
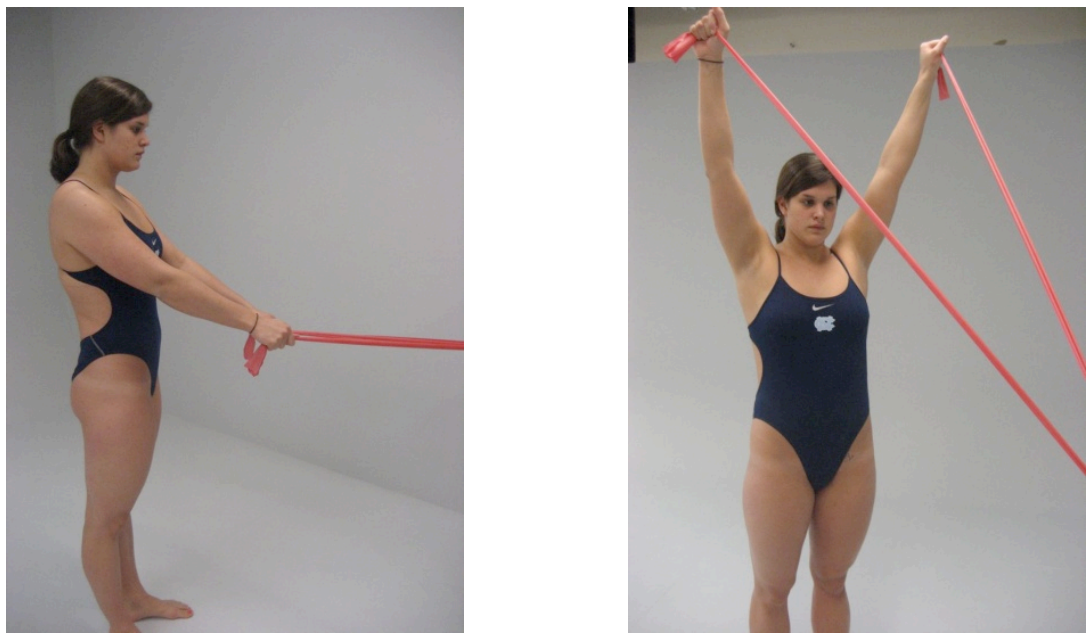
Figure 19: Ts
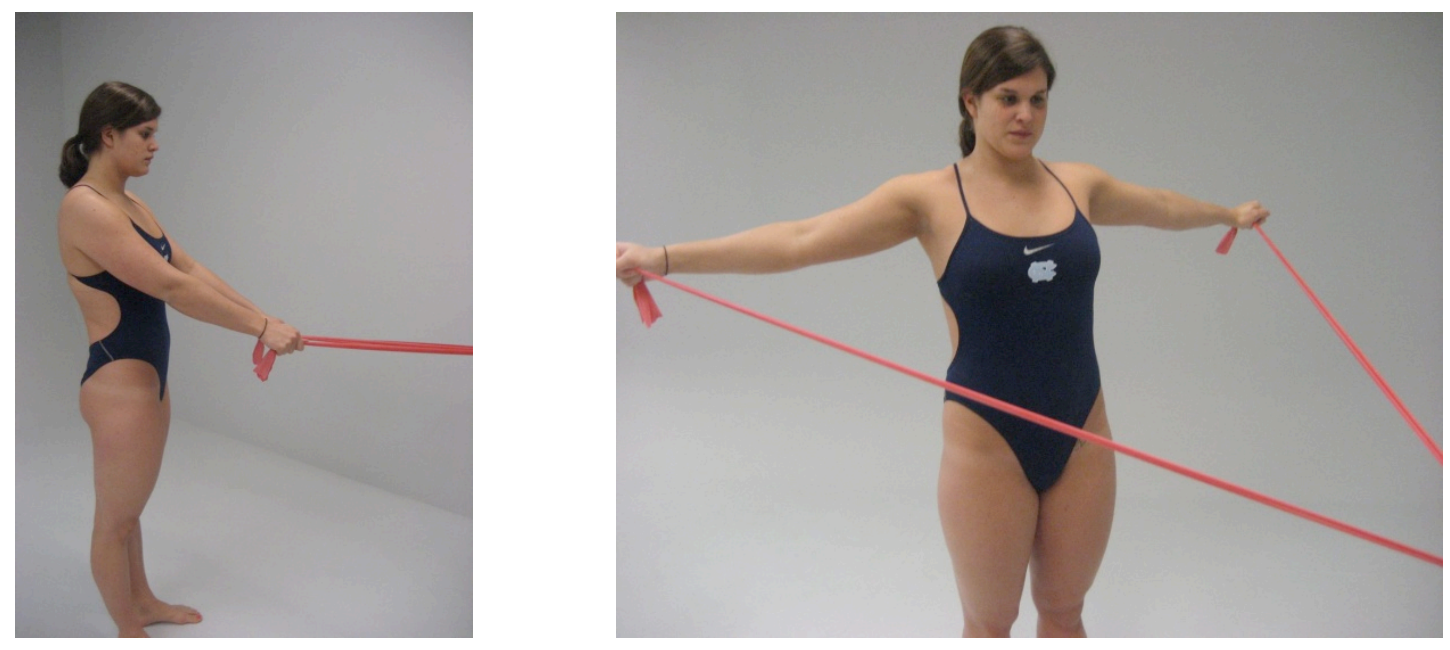

Figure 20: Ws
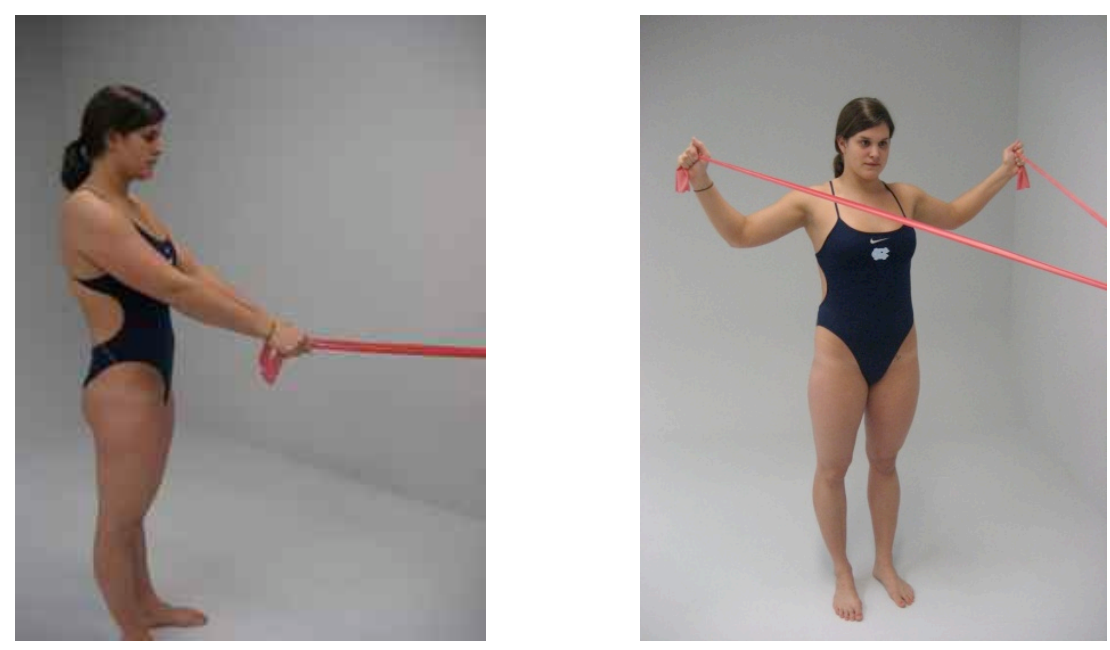

Figure 21: Scapular Punches

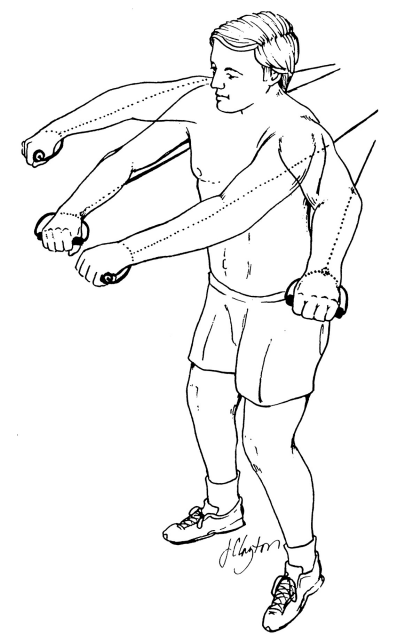


Figure 22: Sleeper Stretch

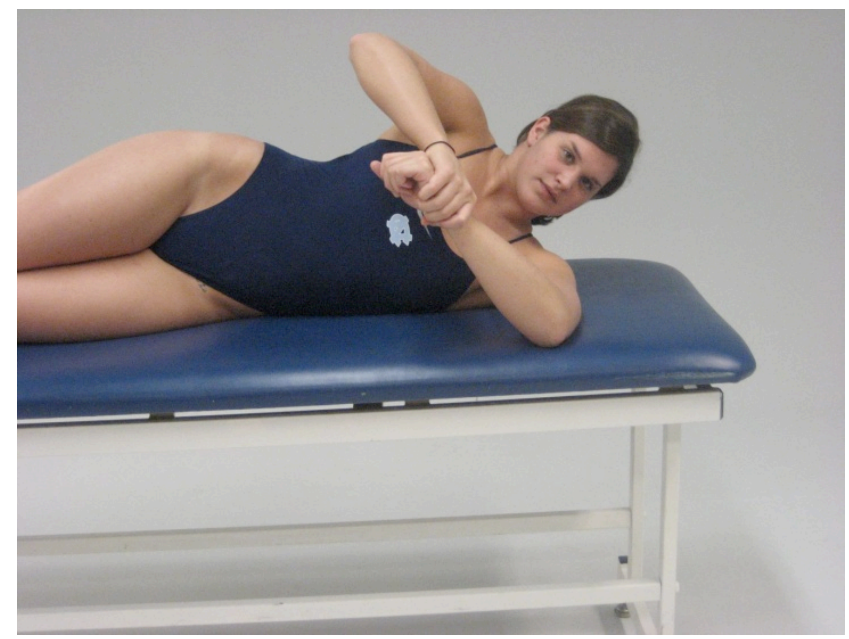

Figure 23: Corner Stretch

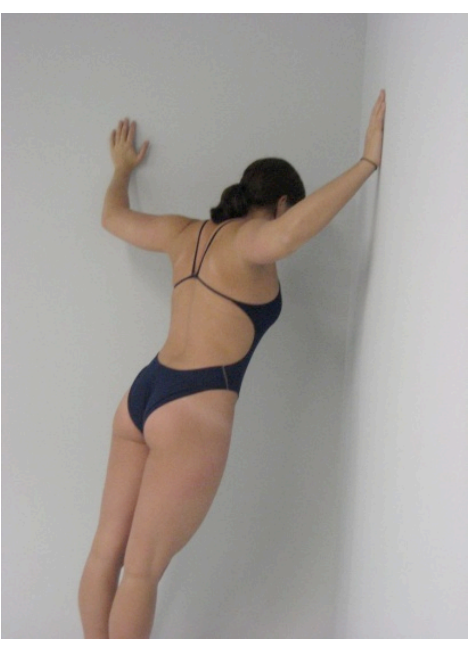


Figure 24: Bony Landmarks of the trunk, scapula and humerus

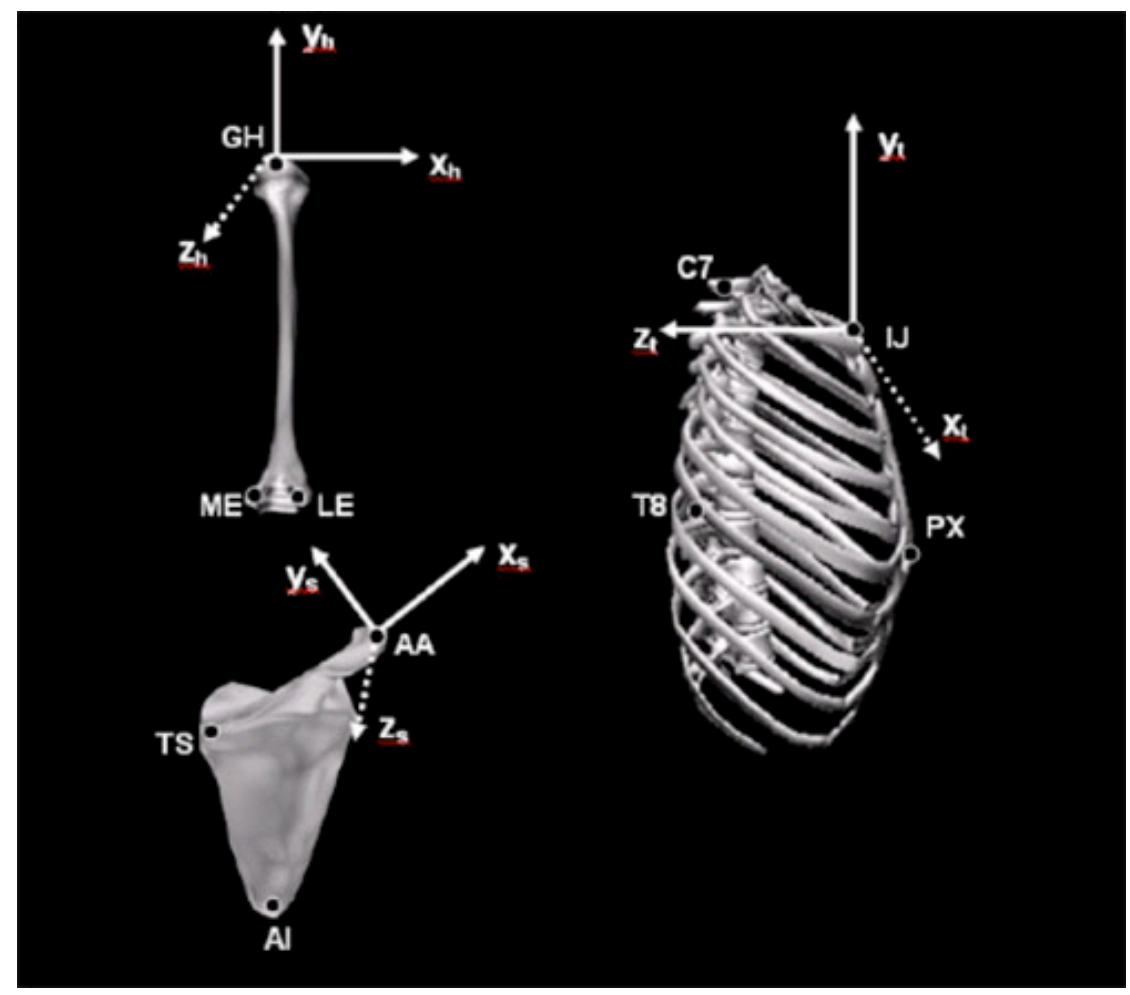

Figure 25: Scapular position and orientation to be measured in the current study

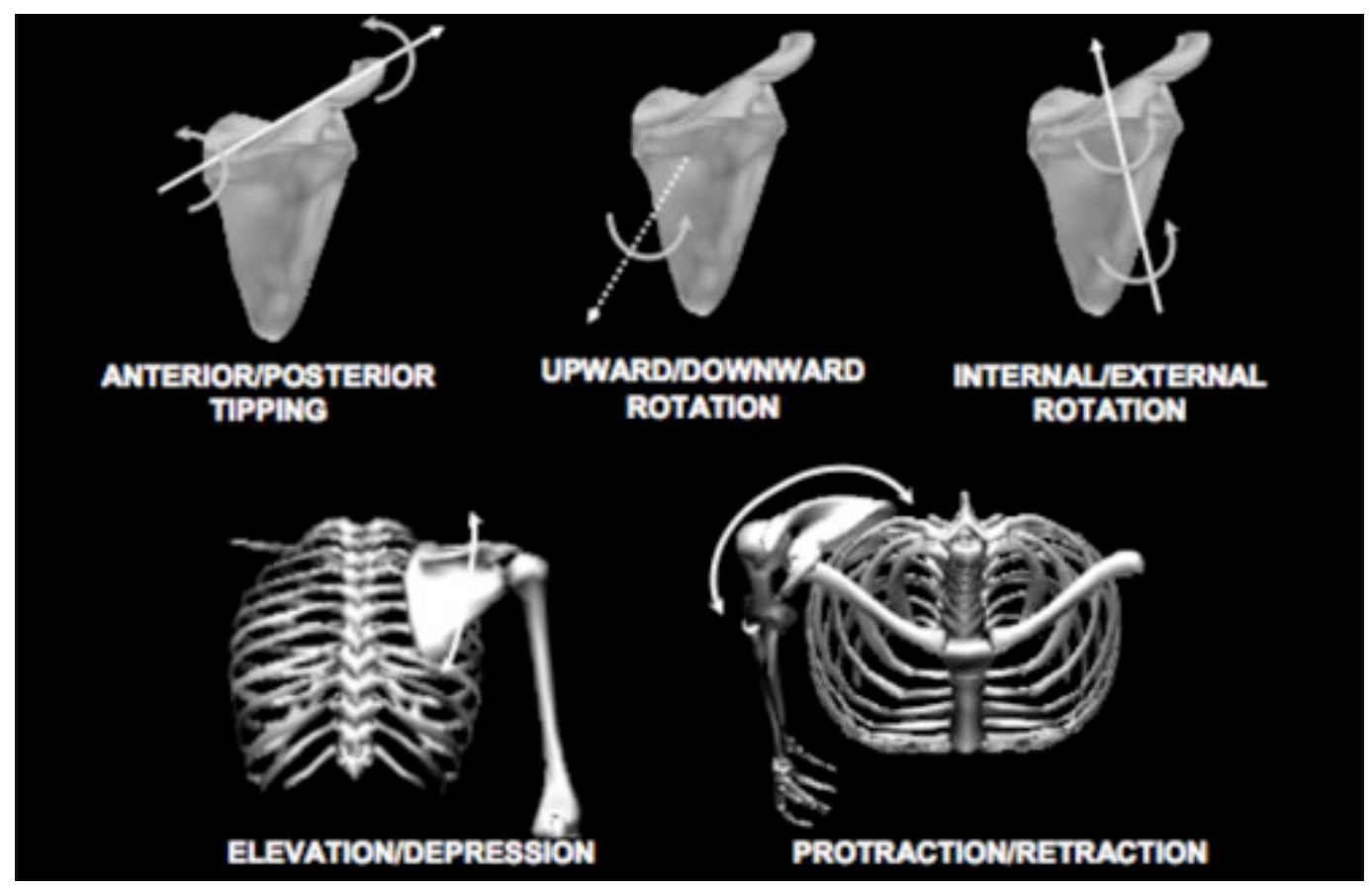


Figure 26: Shoulder flexion strength changes between sessions by group

\section{Shoulder Flexion Strength}

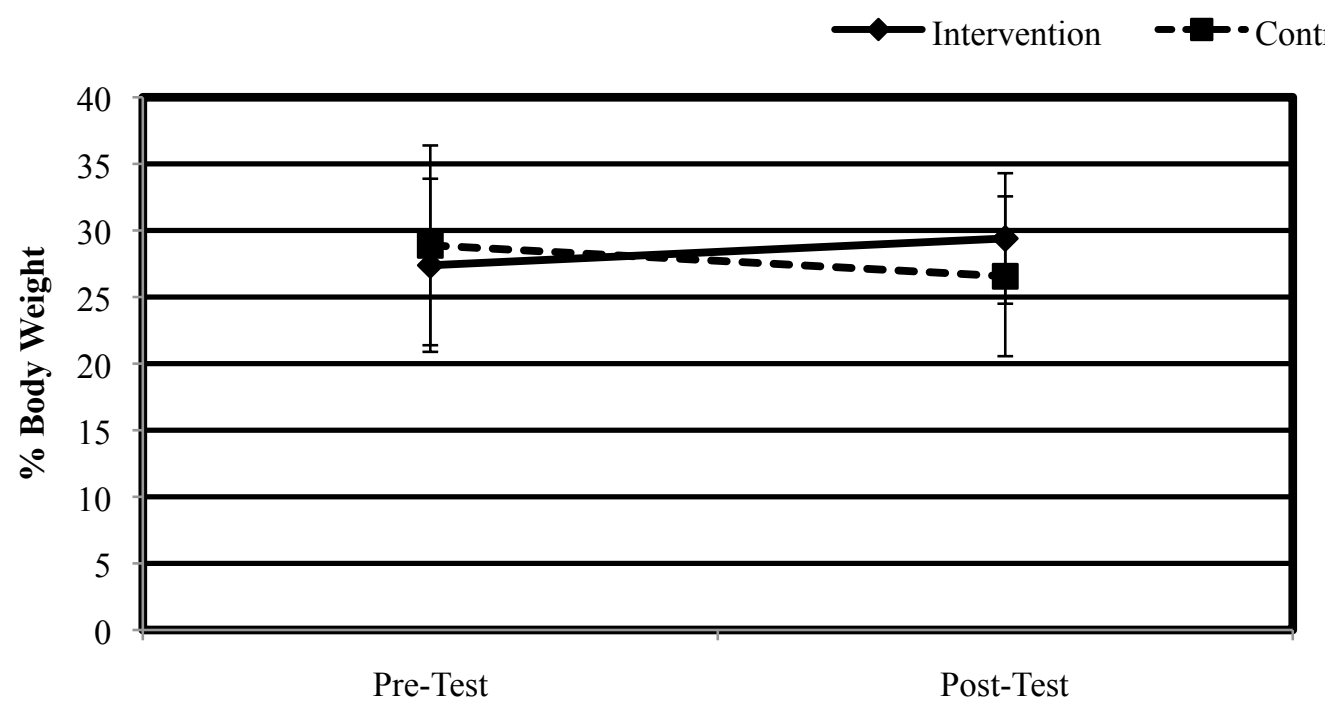

Session

Figure 27: Shoulder abduction strength changes between sessions by group

\section{Shoulder Abduction Strength}

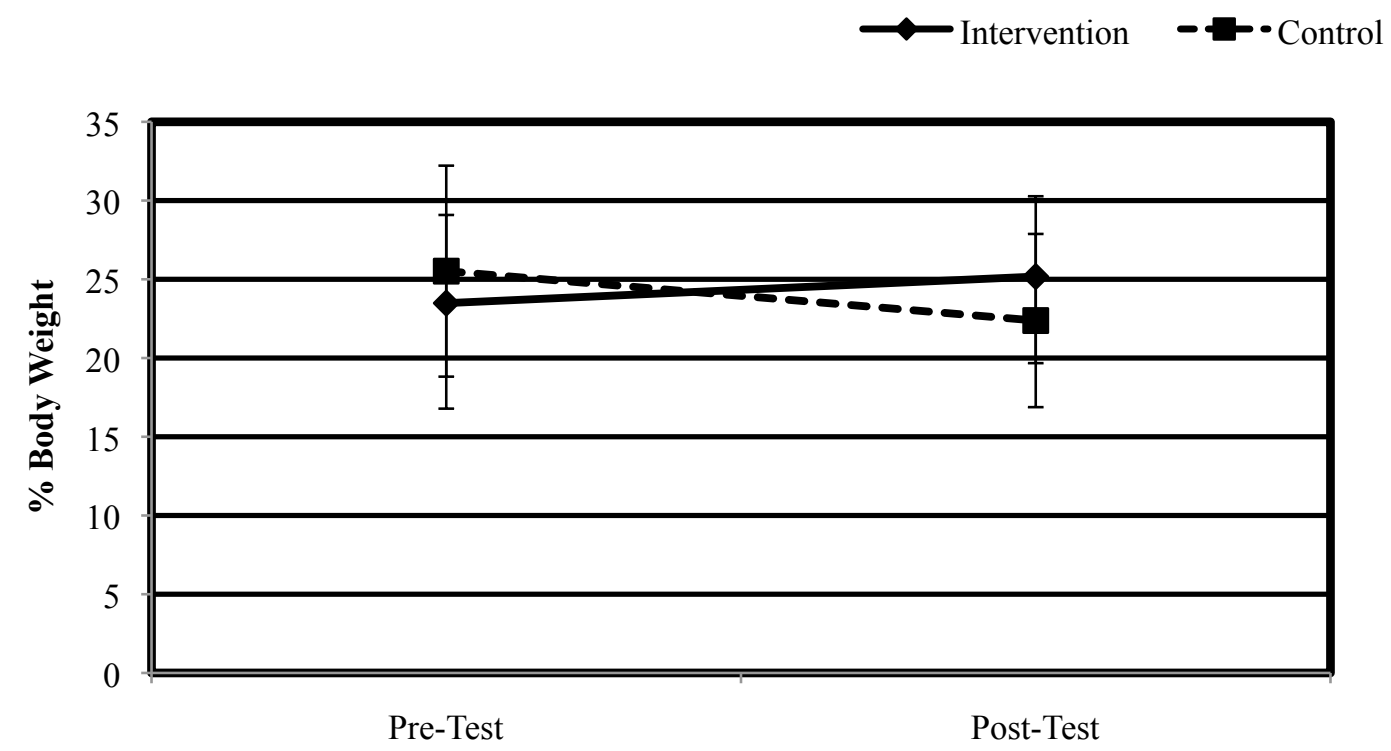

Session 
Figure 28: Shoulder extension strength changes between sessions by group

\section{Shoulder Extension Strength}

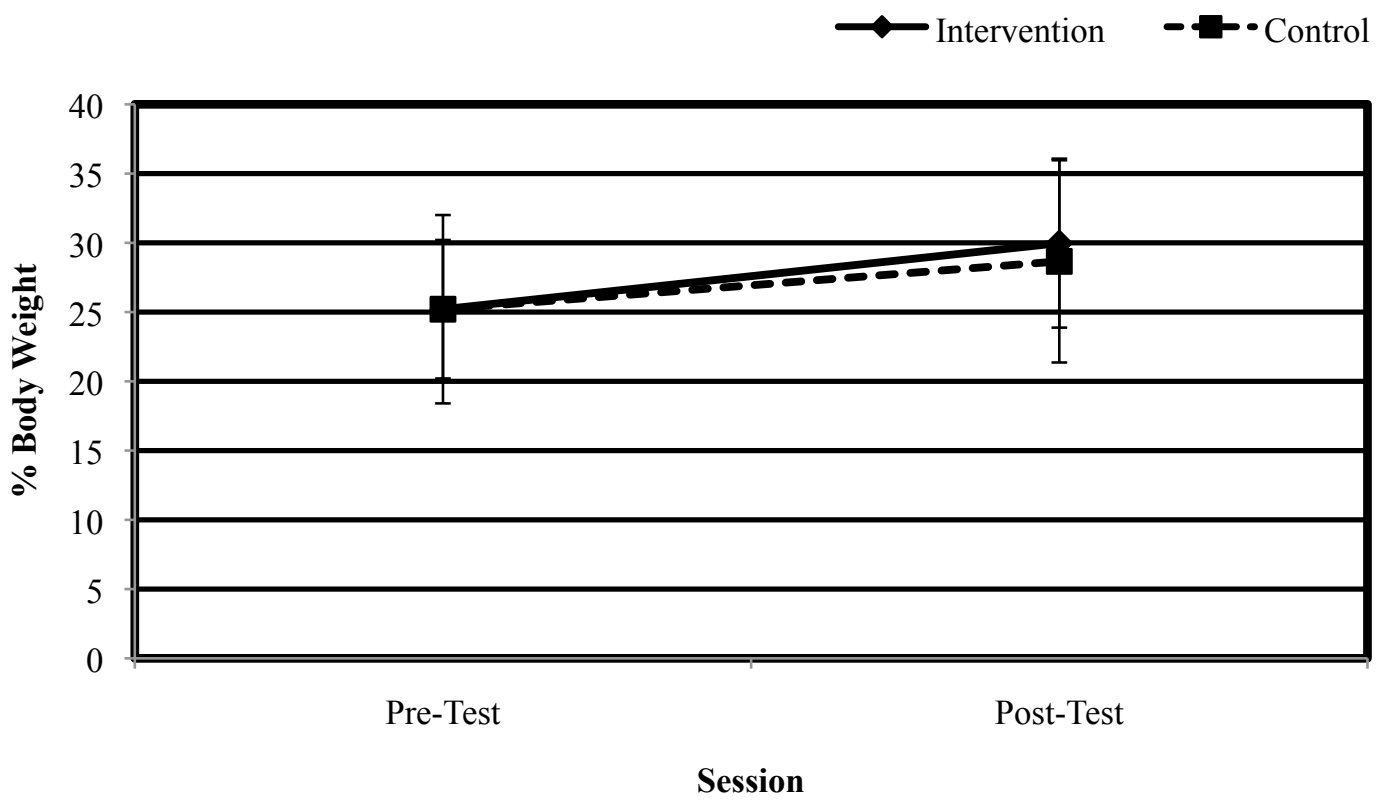

Figure 29: Shoulder internal rotation strength changes between sessions by group

\section{Shoulder Internal Rotation Strength}

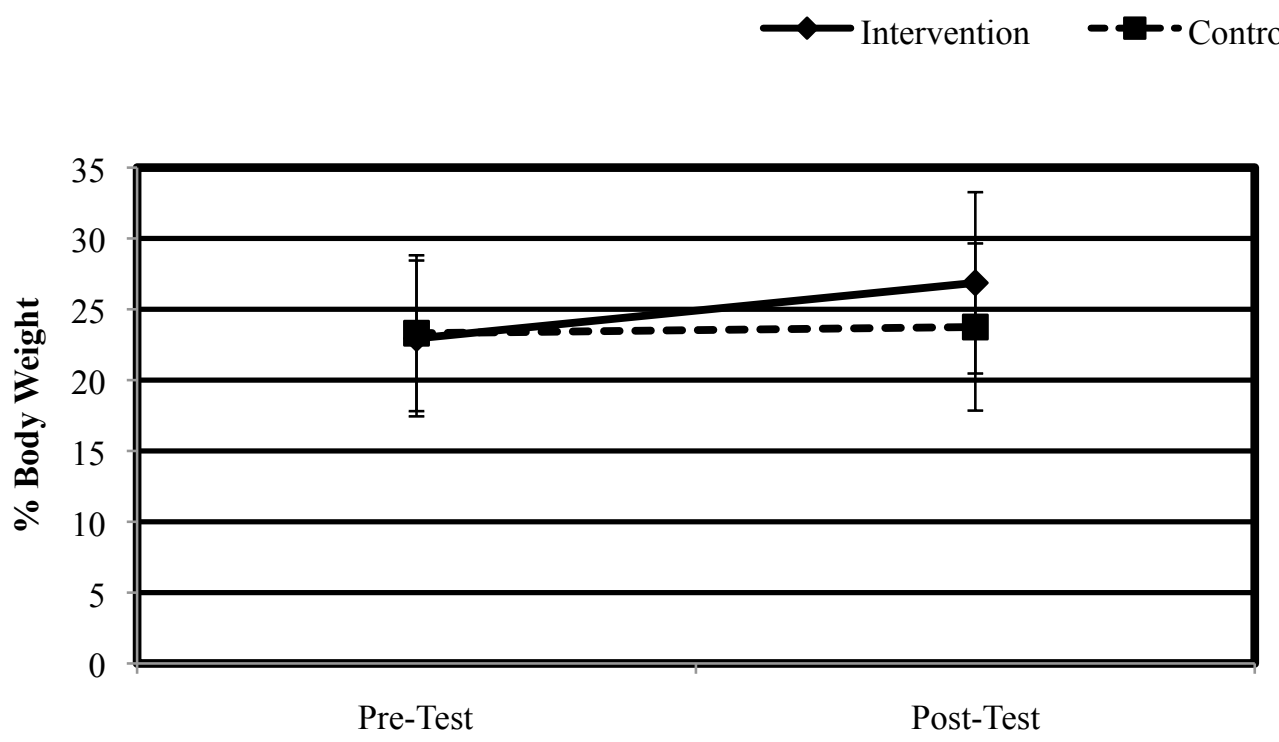

Session 
Figure 30: Shoulder external rotation strength changes between sessions by group

\section{Shoulder External Rotation Strength}

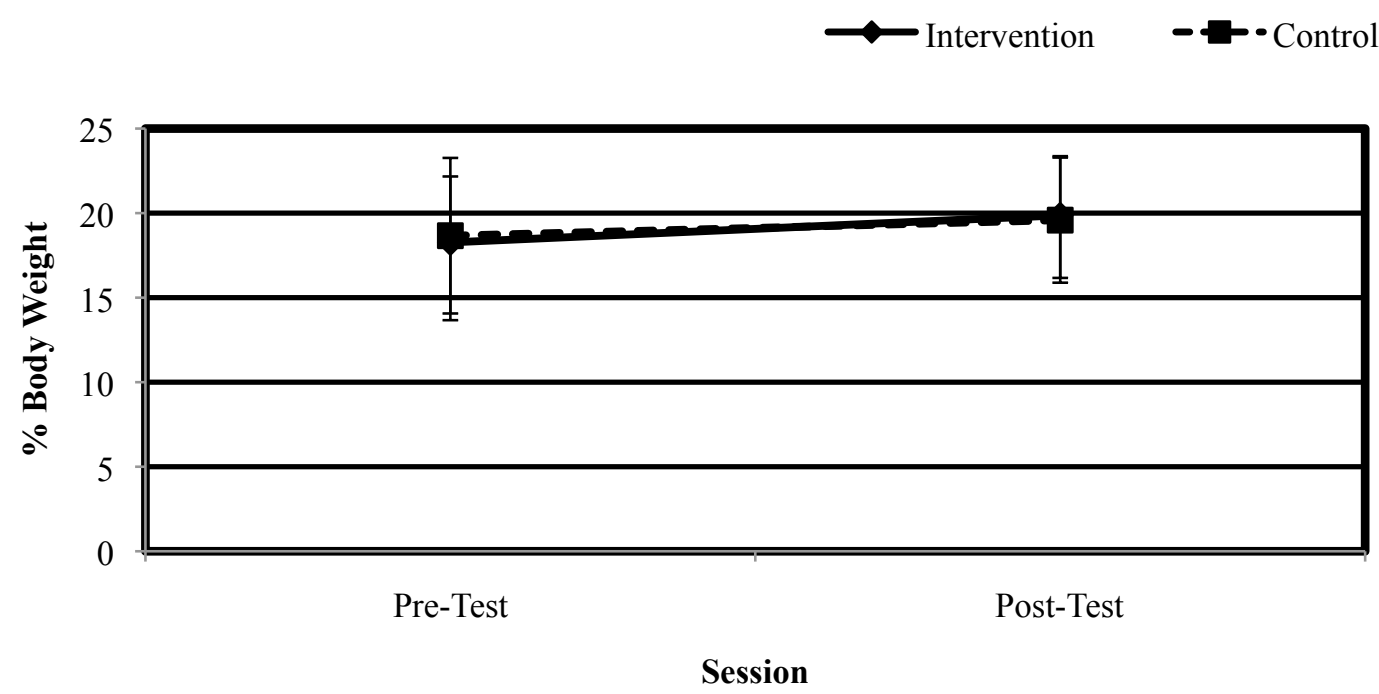

Figure 31: Shoulder adduction strength changes between sessions by group

\section{Shoulder Adduction Strength}

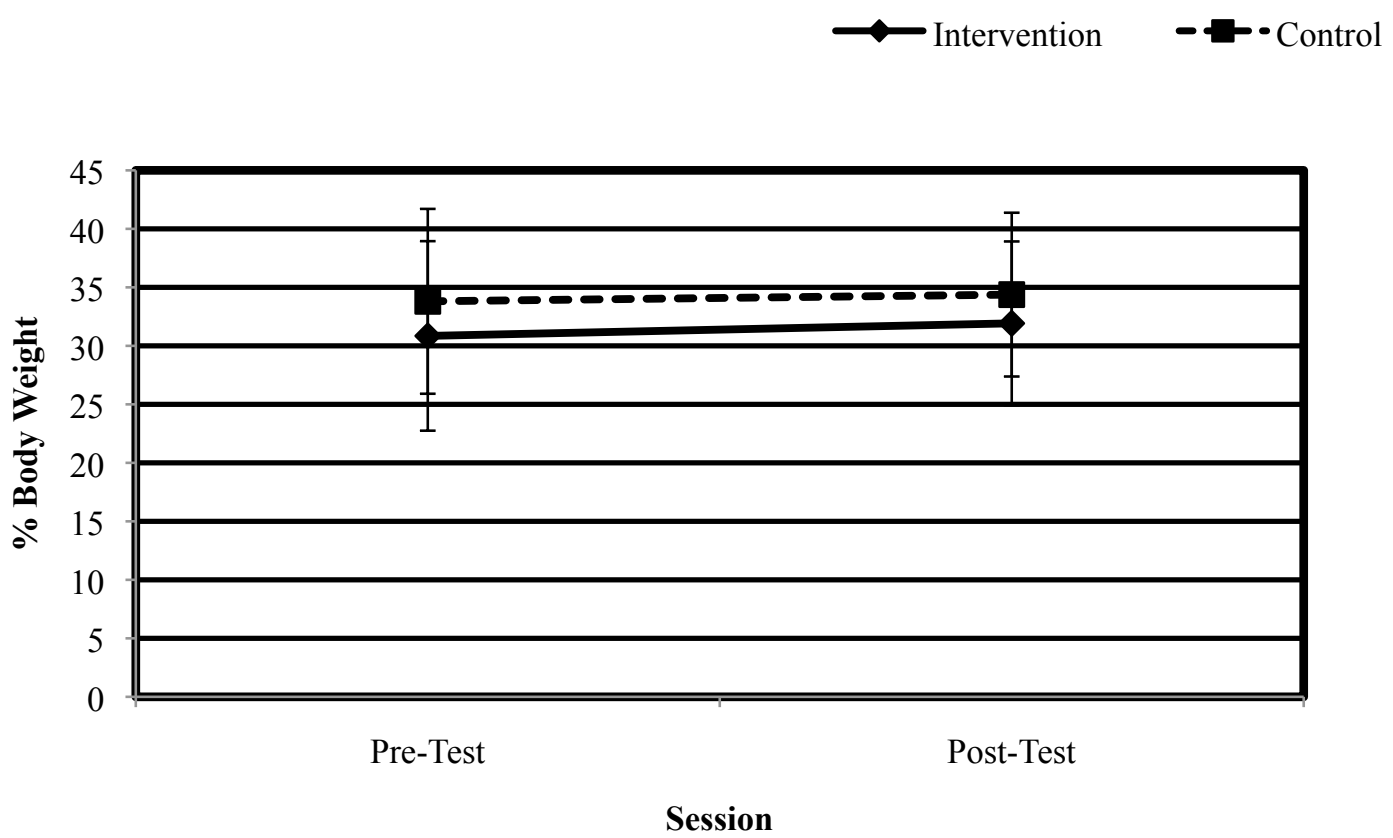


Figure 32: Scapular retraction strength changes between sessions by group

\section{Scapular Retraction Strength}

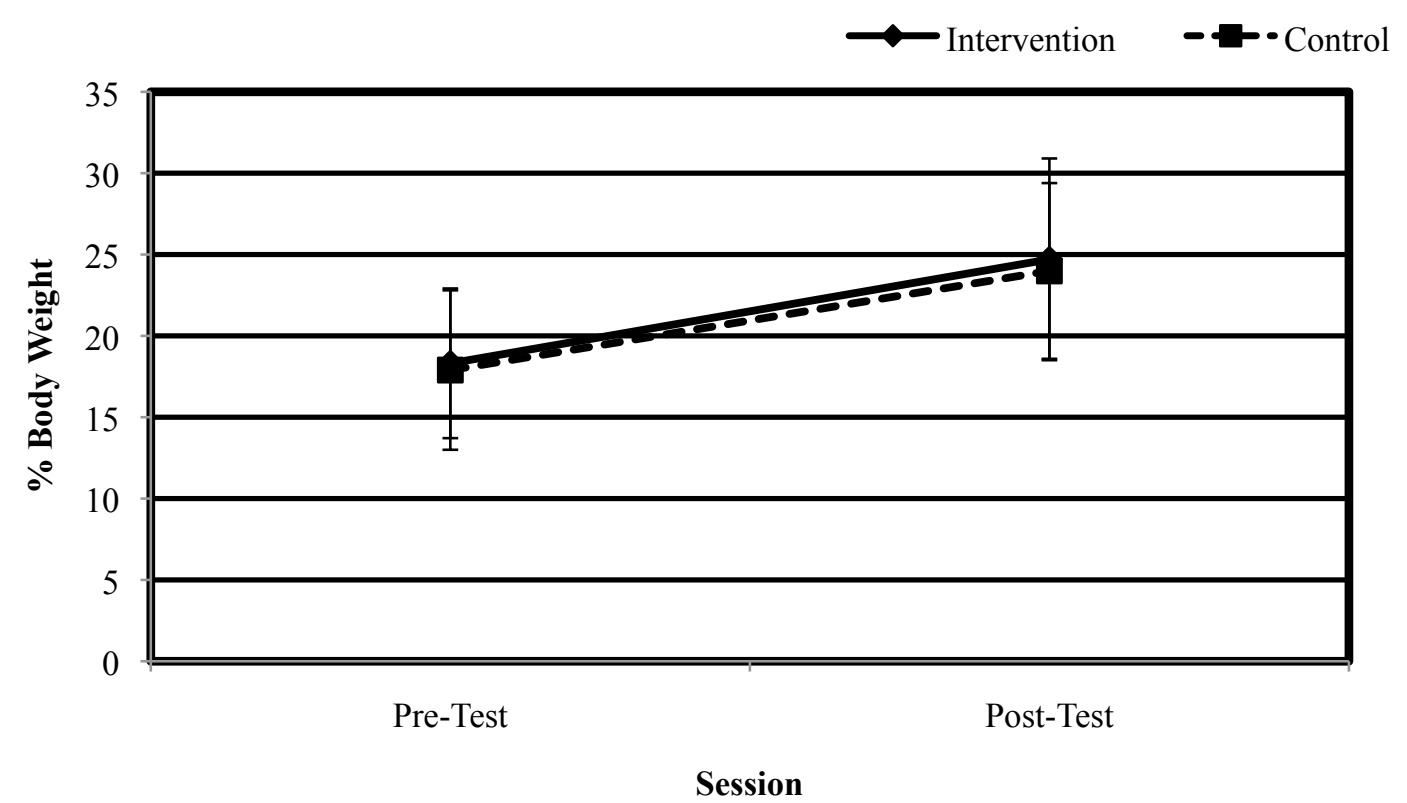

Figure 33: Shoulder retraction with downward rotation strength changes between sessions by group

\section{Scapular Retraction with Downward Rotation Strength}

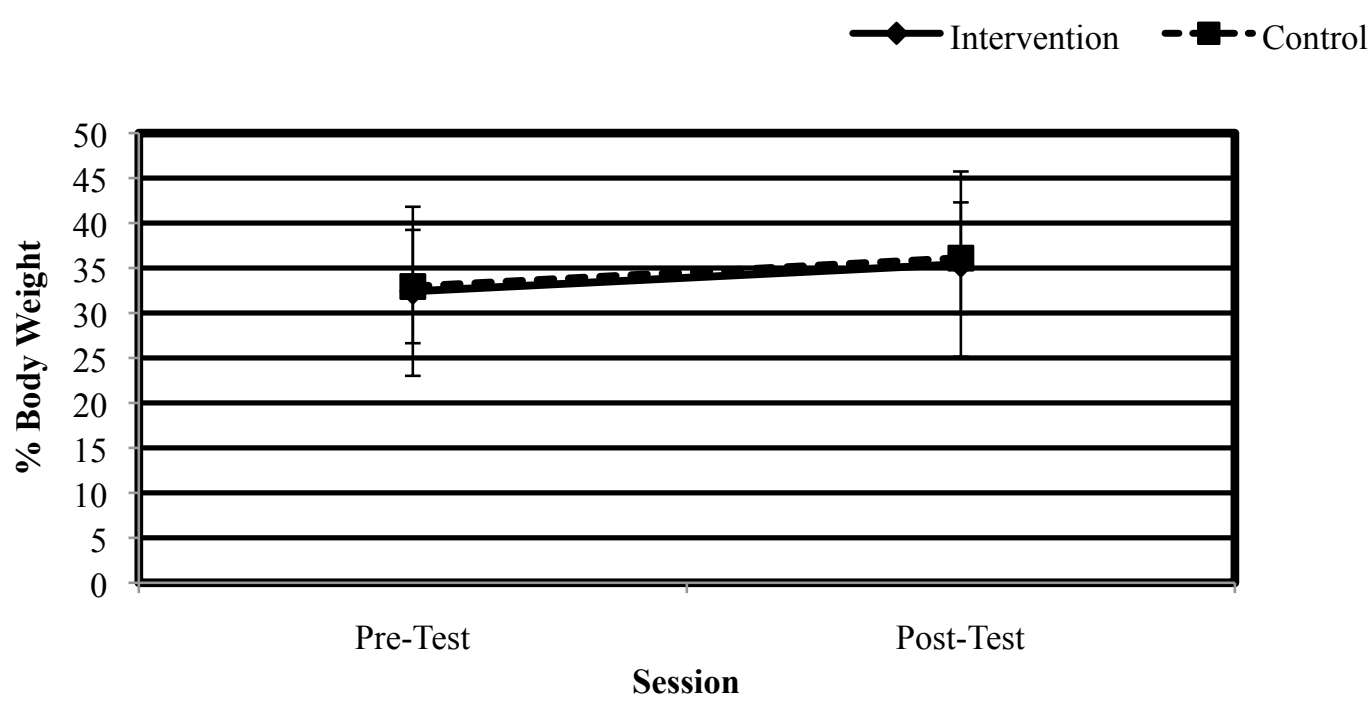


Figure 34: Shoulder retraction with upward rotation strength changes between sessions by group

\section{Scapular Retraction with Upward Rotation Strength}

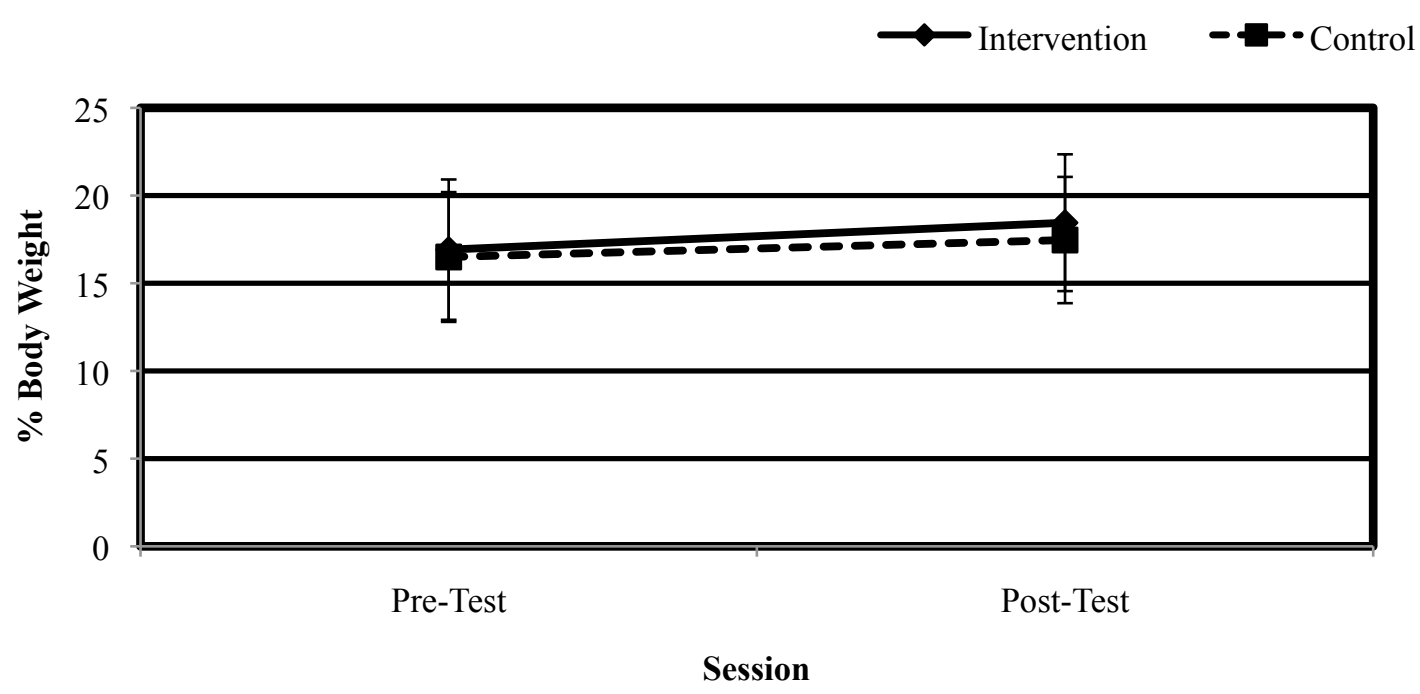


Figure 35: Internal-External Rotation Changes of session, angle, and group

\section{Internal-External Rotation Changes of session, angle, and group}

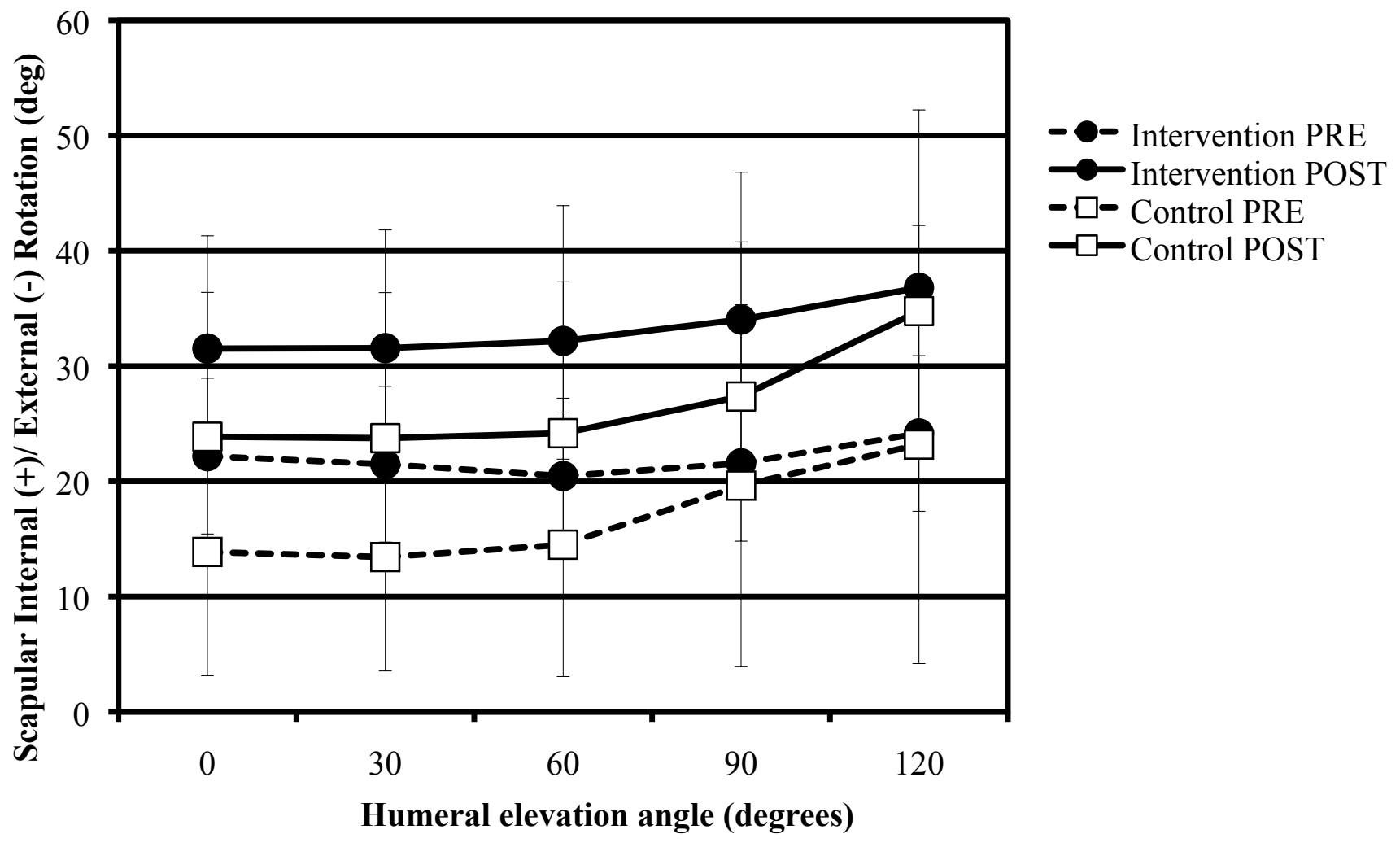


Figure 36: Upward-Downward Rotation Changes of session, angle, and group

Upward-Downward Rotation Changes of session, angle, and group

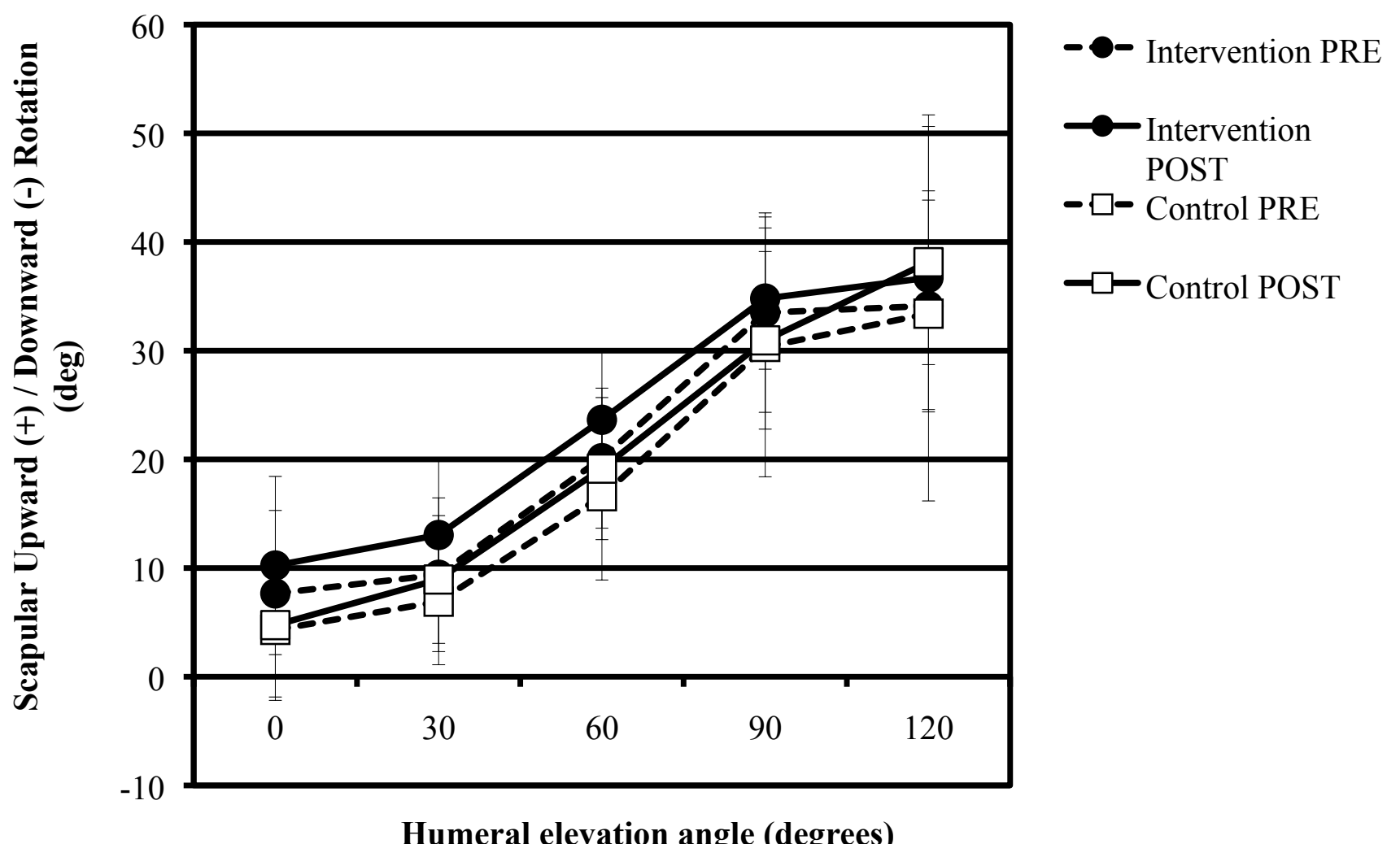


Figure 37: Anterior-Posterior Tipping Changes of session, angle, and group

Anterior-Posterior Tipping Changes of session, angle, and group

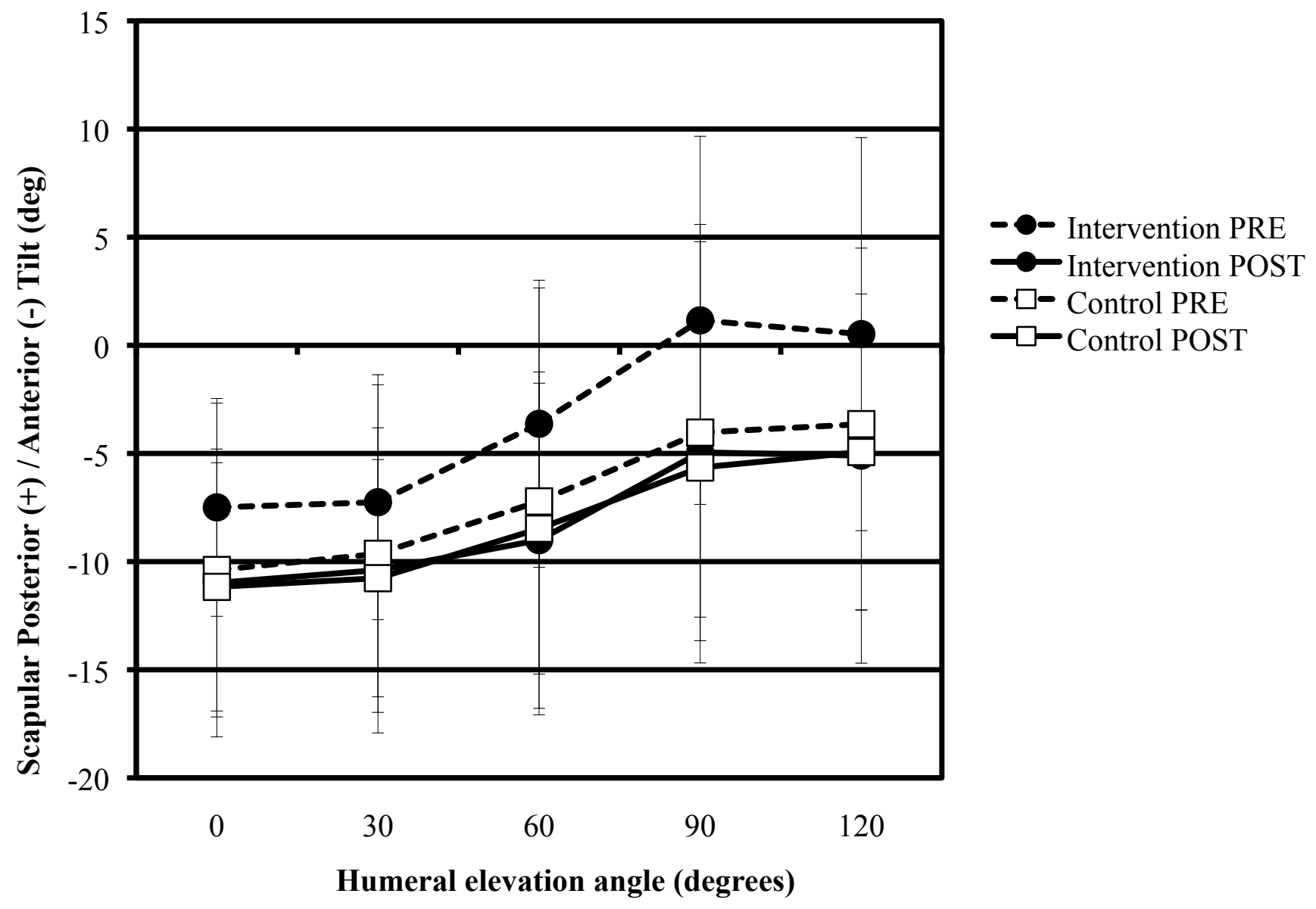


Figure 38: Protraction-Retraction Changes of session, angle, and group

Protraction-Retraction Changes of session, angle, and group

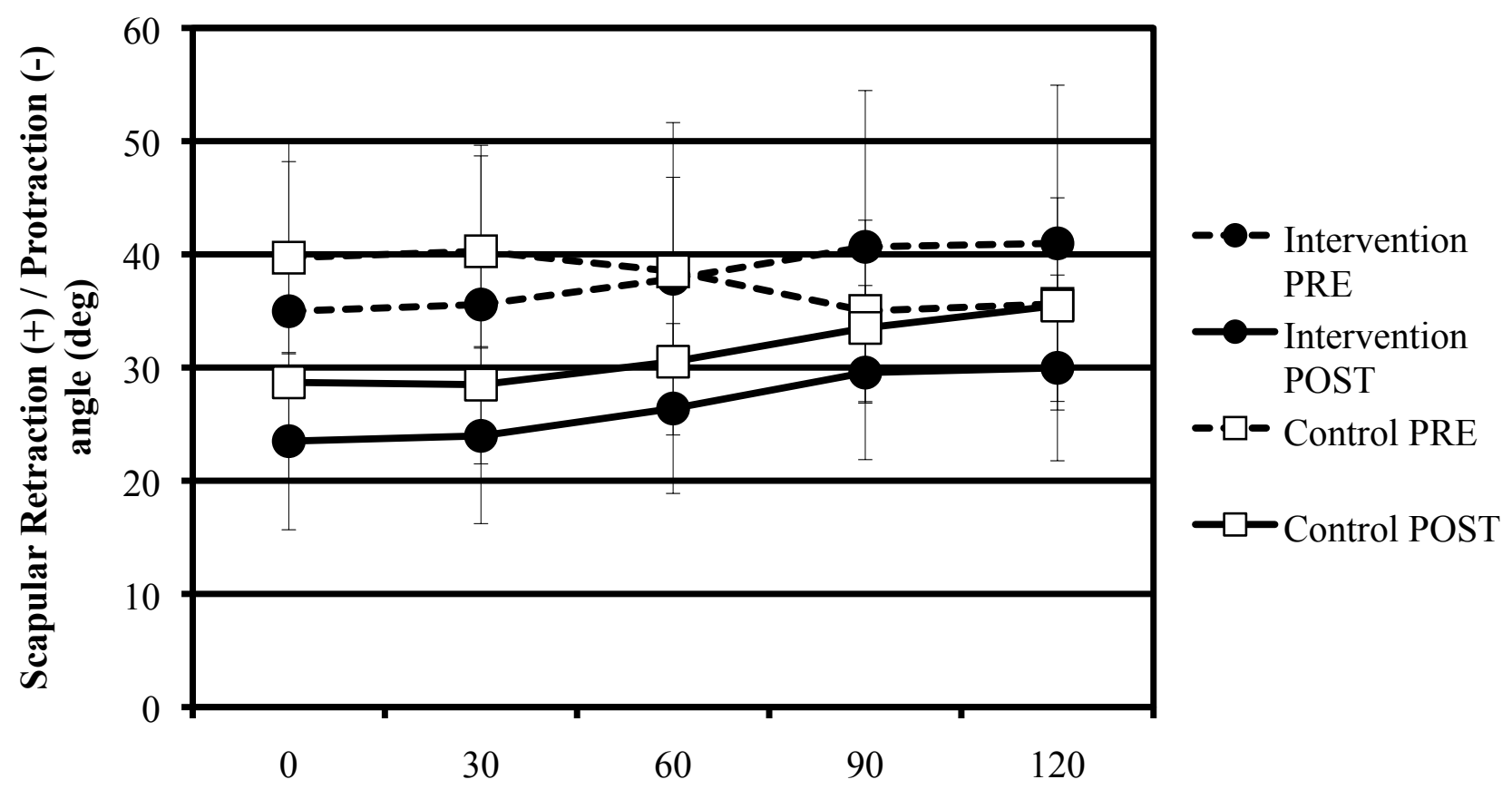

Humeral elevation angle (degrees) 
Figure 39: Elevation-Depression Changes of session, angle, and group

Elevation-Depression Changes of session, angle, and group

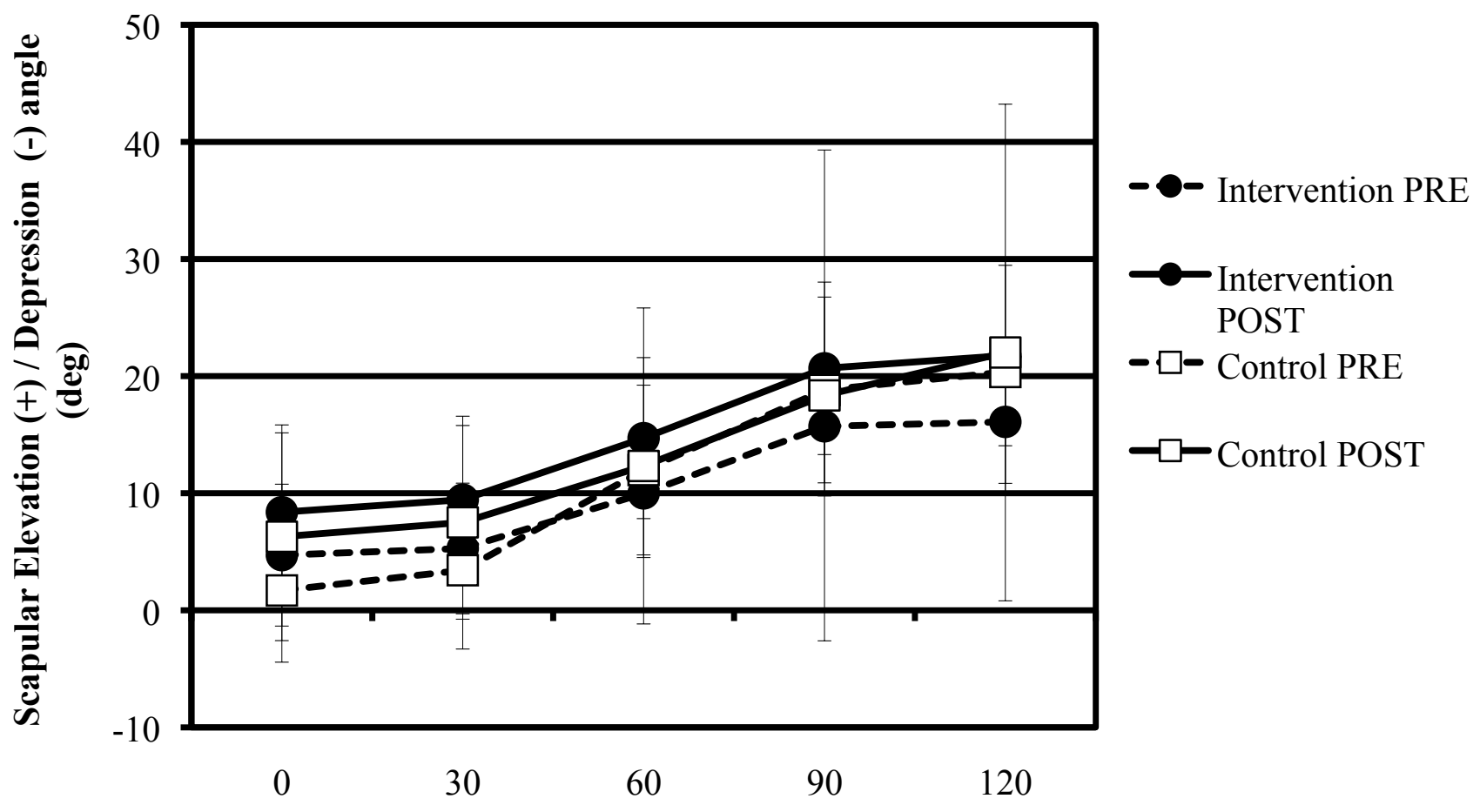

Humeral elevation angle (degrees) 
Figure 40: SANE reporting changes between sessions by group

\section{SANE Reporting}

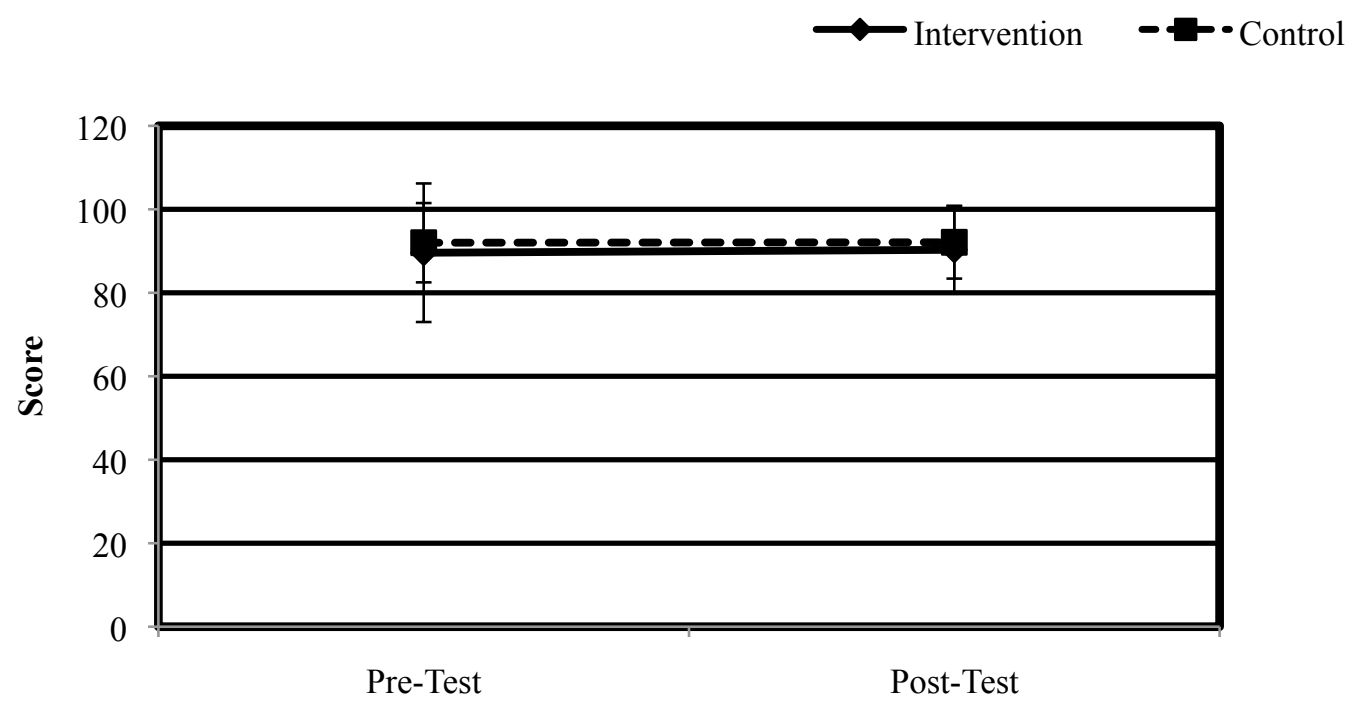

Session

Figure 41: DASH composite score reporting changes between sessions by group

\section{DASH Composite Score Reporting}

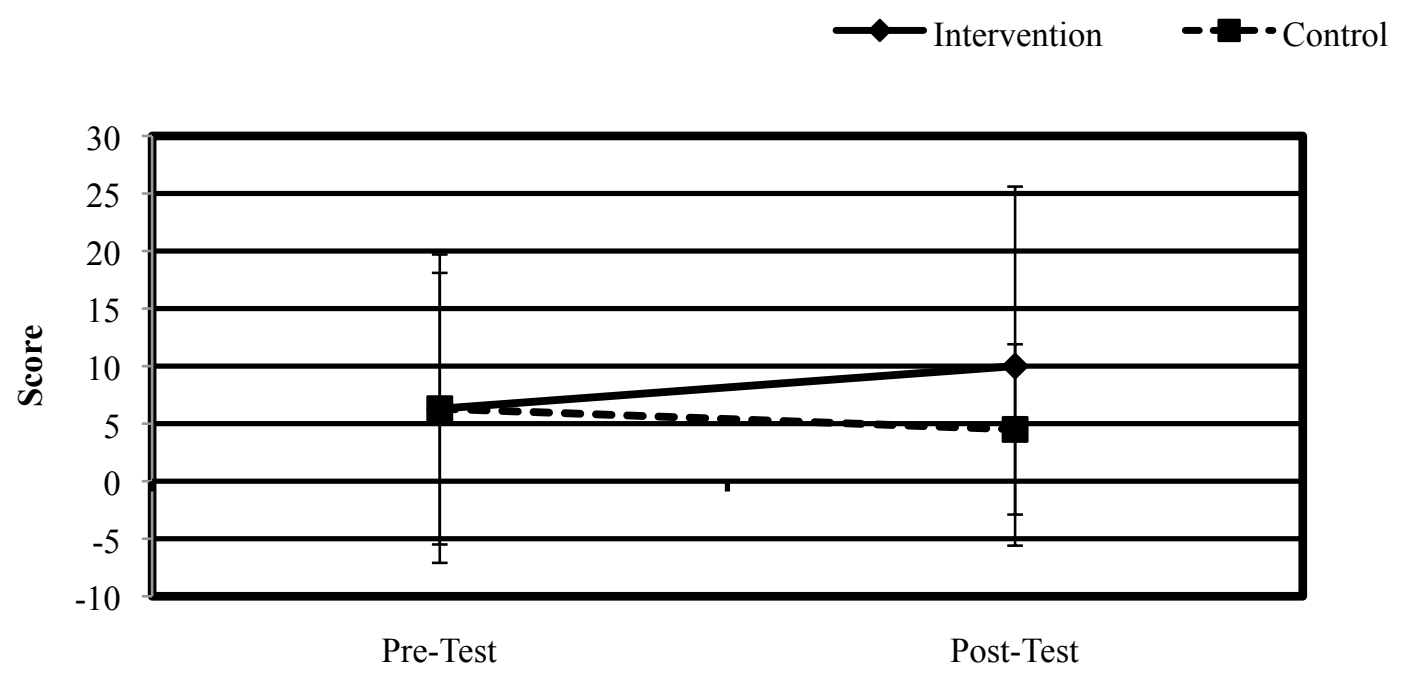

Session 


\section{Tables}

Table 1: Intersession Reliability of Hand Held Dynamometer

\begin{tabular}{|c|c|c|}
\hline $\begin{array}{c}\text { Strength (\%body } \\
\text { mass) }\end{array}$ & Intersession ICC & Intersession SEM \\
\hline Flexion & .987 & .67 \\
\hline Extension & .979 & .89 \\
\hline Abduction & .988 & .69 \\
\hline Adduction & .991 & .79 \\
\hline Internal Rotation & .996 & .52 \\
\hline External Rotation & .987 & .66 \\
\hline Scapular Protraction & .993 & .63 \\
\hline Scapular Retraction & .990 & .49 \\
\hline $\begin{array}{c}\text { Scapular Retraction } \\
\text { with Downward } \\
\text { Rotation }\end{array}$ & .993 & .36 \\
\hline $\begin{array}{c}\text { Scapular Retraction } \\
\text { with Upward } \\
\text { Rotation }\end{array}$ & .982 & 1.24 \\
\hline
\end{tabular}

Table 2: Intrasession and Intertester Reliability and Precision of Electromagnetic

Tracking of Scapular Kinematics

\begin{tabular}{|c|c|c|c|c|}
\hline Scapular Kinematics $\left(^{\circ}\right)$ & $\begin{array}{c}\text { Intrasession } \\
\text { ICC }\end{array}$ & $\begin{array}{c}\text { Intrasession } \\
\text { SEM }\end{array}$ & Intertester ICC & $\begin{array}{c}\text { Intertester } \\
\text { SEM }\end{array}$ \\
\hline $\begin{array}{c}\text { Internal/External } \\
\text { Rotation }\end{array}$ & $.93-.99$ & $.84-1.4^{\circ}$ & $.75-.97$ & $2.1-4.5^{\circ}$ \\
\hline $\begin{array}{c}\text { Anterior/Posterior } \\
\text { Tipping }\end{array}$ & $.97-.98$ & $.89-1.2^{\circ}$ & $.83-.91$ & $2.3-3.4^{\circ}$ \\
\hline $\begin{array}{c}\text { Upward/Downward } \\
\text { Rotation }\end{array}$ & $.91-.98$ & $.73-1.1^{\circ}$ & $.62-.83$ & $1.9-4.3^{\circ}$ \\
\hline Protraction/Retraction & $.80-.96$ & $1.0-2.1^{\circ}$ & $.71-.92$ & $1.4-2.3^{\circ}$ \\
\hline Elevation/Depression & $.85-.98$ & $.71-1.6^{\circ}$ & $.83-.88$ & $1.5-2.0^{\circ}$ \\
\hline
\end{tabular}


Table 3: Bony Landmarks of the thorax, scapula and humerus

\begin{tabular}{|c|l|}
\hline Bony Landmarks & Description of Palpation Point \\
\hline Thorax & Most dorsal point \\
\hline 8th Thoracic Spinous Process (T8) & Most caudal point of sternum \\
\hline Processus xiphoideus (PX) & Most dorsal point \\
\hline 7th Cervical Spinous Process (C7) & $\begin{array}{l}\text { Most cranial point of the sternum } \\
\text { (suprasternal notch) }\end{array}$ \\
\hline Incisura jugularis (IJ) & $\begin{array}{l}\text { Junction between the acromion process and } \\
\text { the most lateral point of the clavicle }\end{array}$ \\
\hline Scapula & $\begin{array}{l}\text { Midpoint of triangular surface on the medial } \\
\text { border of the scapula in line with the scapular } \\
\text { spine }\end{array}$ \\
\hline Acromio-clavicular joint (AC) & Most caudal point of scapula \\
\hline Trigonum spinae (TS) & Most medial point on the medial epicondyle \\
\hline Angulus inferior (AI) & Most lateral point on the lateral epicondyle \\
\hline Humerus & \\
\hline Medial epicondyle (ME) & Lateral epicondyle (LE) \\
\hline Glenohumeral joint center (GH)* & \\
\hline
\end{tabular}

* The glenohumeral joint center was not palpated but rather estimated with a least squares algorithm for the point on the humerus which moves the least during several short arc humeral movements (Harryman, Sidles et al. 1990; Stokdijk, Nagels et al. 2000). 
Table 4: Definition of Local Coordinate Systems

\section{Local Coordinate System}

$\underline{\text { Axis }}$

Thorax

Scapula

Humerus

\section{$\underline{\text { Definition }}$}

Vector from the midpoint of $\mathrm{PX}$ and $\mathrm{t} 8$ to the midpoint between IJ and C7

Vector perpendicular to the plane fitted by midpoint of PX and T8, the midpoint of IJ and $\mathrm{C} 7$ and $\mathrm{IJ}$ Vector perpendicular to $\mathrm{Y}_{\mathrm{t}}$ and $Z_{t}$

IJ

Vector from TS to AA

Vector perpendicular to the plane fitted by TS, AA and AI (scapular plane) Vector perpendicular to $X_{s}$ and $\mathrm{Y}_{\mathrm{s} \text {, }}$

AA

Vector from midpoint of ME and LE to GH Vector perpendicular to the plane fitted by GH, ME and LE

Perpendicular to $\mathrm{X}_{\mathrm{h}}$ and $\mathrm{Y}_{\mathrm{h}}$ $\mathrm{GH}$ 
Table 5: Summary of Exercises and Stretches to be performed in the current study

\begin{tabular}{|c|c|c|}
\hline Exercise & $\begin{array}{l}\text { Muscles High in EMG } \\
\text { Activation }\end{array}$ & EMG Studies \\
\hline Shoulder Flexion & $\begin{array}{l}\text { AD, Rhom, } \\
\text { SA, Sub, TM }\end{array}$ & $\begin{array}{l}\text { Moseley, Jobe et al. 1992; } \\
\text { Myers, Pasquale et al. 2005; } \\
\text { Cools, Dewitte et al. 2007) }\end{array}$ \\
\hline Shoulder Extension & $\begin{array}{l}\text { Lat, Rhom, } \\
\text { Sub, Tri, TM }\end{array}$ & $\begin{array}{l}\text { (Myers, Pasquale et al. 2005; } \\
\text { Cools, Dewitte et al. 2007) }\end{array}$ \\
\hline IR at $90^{\circ}$ & $\begin{array}{l}\text { LT, Rhom, } \\
\text { SA, Sub, TM }\end{array}$ & (Myers, Pasquale et al. 2005) \\
\hline ER at $90^{\circ}$ & $\begin{array}{l}\text { LT, Rhom, } \\
\text { SA, Sub, } \\
\text { Supra, TM }\end{array}$ & (Myers, Pasquale et al. 2005) \\
\hline Throwing acceleration & $\begin{array}{l}\text { LT, Rhom, } \\
\text { SA, Sub, TM }\end{array}$ & $\begin{array}{l}\text { (Cordasco, Wolfe et al. 1996; } \\
\text { Myers, Pasquale et al. 2005) }\end{array}$ \\
\hline Throwing deceleration & $\begin{array}{l}\text { LT, Rhom, Sub, Supra, TM, } \\
\text { LT, UT }\end{array}$ & $\begin{array}{l}\text { (Moseley, Jobe et al. 1992; } \\
\text { Cordasco, Wolfe et al. 1996; } \\
\text { Myers, Pasquale et al. 2005) }\end{array}$ \\
\hline Low Rows & Rhom, Sub, TM & $\begin{array}{l}\text { (Moseley, Jobe et al. 1992; } \\
\text { Hintermeister, Lange et al. } \\
\text { 1998; Myers, Pasquale et al. } \\
\text { 2005; Cools, Dewitte et al. } \\
\text { 2007) }\end{array}$ \\
\hline Scapular Punches & Rhom, SA, Sub TM & $\begin{array}{l}\text { (Hintermeister, Lange et al. } \\
\text { 1998; Decker, Hintermeister et } \\
\text { al. 1999; Ekstrom, Donatelli et } \\
\text { al. 2003; Myers, Pasquale et al. } \\
\text { 2005) }\end{array}$ \\
\hline Ys & $\mathrm{LT}, \mathrm{MT}, \mathrm{SA}$ & $\begin{array}{l}\text { (Ekstrom, Donatelli et al. 2003; } \\
\text { Reinold, Macrina et al. 2007) }\end{array}$ \\
\hline Ts & Infra, MT, SA, TM, UT & (Ekstrom, Donatelli et al. 2003) \\
\hline Ws & Infra, LT, Rhom, Supra, TM & (Ekstrom, Donatelli et al. 2003) \\
\hline Sleeper Stretch & $\mathrm{N} / \mathrm{A}$ & (McClure, Balaicuis et al. 2007) \\
\hline Crossbody Stretch & $\mathrm{N} / \mathrm{A}$ & (McClure, Balaicuis et al. 2007) \\
\hline Corner Stretch & $\mathrm{N} / \mathrm{A}$ & (Borstad and Ludewig 2006) \\
\hline
\end{tabular}

** $\mathrm{AD}=$ Anterior Deltoid; Infra= Infraspinatus; Lat= Latissimus Dorsi; LT= Lower Trap; MT= Middle Trap; Rhom=Rhomboids; SA=Serratus Anterior; Sub=Subscapularis; Supra $=$ Supraspinatus; $\mathrm{TM}=$ Teres Minor; Tri= Triceps; UT= Upper Trapezius 
Table 6: Subject Demographics

\begin{tabular}{ccc}
\hline & Intervention & Control \\
\hline Number of Subjects (n) & 20 & 17 \\
Males / Females & $10 / 10$ & $8 / 9$ \\
Age (yrs) & $19.2 \pm 1.2$ & $19.4 \pm 1.2$ \\
Mass (kg) & $73.1 \pm 9.9$ & $72.8 \pm 12.4$ \\
Height (cm) & $177.5 \pm 9.8$ & $178.1 \pm 8.7$ \\
\hline
\end{tabular}

Table 7: Means and standard deviations (SD) of the shoulder and scapular stabilizer strength (\% body mass (BM)) before and after the intervention and the change score

\begin{tabular}{|c|c|c|c|c|c|c|}
\hline & \multicolumn{3}{|c|}{ Intervention } & \multicolumn{3}{|c|}{ Control } \\
\hline & $\begin{array}{c}\text { Pre } \\
\text { Mean } \pm \text { SD }\end{array}$ & $\begin{array}{c}\text { Post } \\
\text { Mean } \pm \text { SD }\end{array}$ & $\begin{array}{c}\text { Change } \\
\text { Mean } \pm \text { SD }\end{array}$ & $\begin{array}{c}\text { Pre } \\
\text { Mean } \pm \text { SD }\end{array}$ & $\begin{array}{c}\text { Post } \\
\text { Mean } \pm \text { SD }\end{array}$ & $\begin{array}{c}\text { Change } \\
\text { Mean } \pm \text { SD }\end{array}$ \\
\hline Flexion (\%BM) & $27.4 \pm 6.5$ & $29.4 \pm 4.9$ & $2.0 \pm 5.0$ & $28.9 \pm 7.5$ & $26.6 \pm 6.0$ & $-2.3 \pm 5.8$ \\
\hline Extension (\%BM) & $25.2 \pm 5.0$ & $29.9 \pm 6.1$ & $4.7 \pm 6.9$ & $25.2 \pm 6.8$ & $28.7 \pm 7.3$ & $3.5 \pm 9.9$ \\
\hline $\begin{array}{l}\text { External } \\
\text { Rotation (\%BM) }\end{array}$ & $18.2 \pm 3.9$ & $19.8 \pm 3.5$ & $1.6 \pm 3.8$ & $18.7 \pm 4.6$ & $19.6 \pm 3.7$ & $0.9 \pm 4.3$ \\
\hline $\begin{array}{l}\text { Internal } \\
\text { Rotation (\%BM) }\end{array}$ & $22.9 \pm 5.5$ & $26.9 \pm 6.4$ & $4.0 \pm 7.1$ & $23.3 \pm 5.5$ & $23.7 \pm 5.9$ & $0.4 \pm 7.1$ \\
\hline Abduction (\%BM) & $23.5 \pm 5.6$ & $25.2 \pm 5.1$ & $1.7 \pm 6.3$ & $25.5 \pm 6.7$ & $22.4 \pm 5.5$ & $-3.1 \pm 4.8$ \\
\hline Adduction (\%BM) & $30.8 \pm 8.1$ & $31.9 \pm 7.0$ & $1.1 \pm 7.4$ & $33.8 \pm 7.9$ & $34.4 \pm 7.0$ & $0.6 \pm 8.2$ \\
\hline Retraction (\%BM) & $18.3 \pm 4.6$ & $24.7 \pm 6.2$ & $6.4 \pm 4.9$ & $17.9 \pm 4.9$ & $24.0 \pm 5.4$ & $6.1 \pm 5.3$ \\
\hline $\begin{array}{l}\text { Retraction with } \\
\text { Downward } \\
\text { Rotation (\%BM) }\end{array}$ & $32.4 \pm 9.4$ & $35.4 \pm 10.3$ & $3.2 \pm 11.6$ & $32.9 \pm 6.3$ & $36.1 \pm 6.2$ & $3.2 \pm 7.0$ \\
\hline $\begin{array}{l}\text { Retraction with } \\
\text { Upward } \\
\text { Rotation (\%BM) }\end{array}$ & $16.9 \pm 4.0$ & $18.5 \pm 3.9$ & $1.6 \pm 4.1$ & $16.5 \pm 3.7$ & $17.5 \pm 3.6$ & $1.0 \pm 3.7$ \\
\hline
\end{tabular}


Table 8: Means and standard deviations (SD) of the Scapular Kinematics during humeral elevation task before and after the intervention and the change score

\begin{tabular}{|c|c|c|c|c|c|c|}
\hline & \multicolumn{3}{|c|}{ Intervention } & \multicolumn{3}{|c|}{ Control } \\
\hline & Pre & Post & Change & Pre & Post & Change \\
\hline & Mean \pm SD & Mean \pm SD & Mean \pm SD & Mean \pm SD & Mean \pm SD & Mean \pm SD \\
\hline \multicolumn{7}{|l|}{ Internal/external rotation $\left({ }^{\circ}\right)$} \\
\hline $0^{\circ}$ humeral elevation & $22.2 \pm 6.8$ & $31.5 \pm 9.8$ & $9.3 \pm 11.9$ & $13.9 \pm 10.7$ & $23.9 \pm 12.5$ & $10.0 \pm 14.0$ \\
\hline $30^{\circ}$ humeral elevation & $21.5 \pm 6.7$ & $31.6 \pm 9.8$ & $10.1 \pm 10.7$ & $13.4 \pm 9.9$ & $23.7 \pm 12.6$ & $10.3 \pm 11.3$ \\
\hline $60^{\circ}$ humeral elevation & $20.5 \pm 7.2$ & $32.2 \pm 10.3$ & $11.8 \pm 11.8$ & $14.5 \pm 11.4$ & $24.2 \pm 13.1$ & $9.7 \pm 12.0$ \\
\hline $90^{\circ}$ humeral elevation & $21.6 \pm 8.4$ & $34.0 \pm 11.7$ & $12.4 \pm 12.1$ & $19.6 \pm 15.7$ & $27.4 \pm 13.4$ & $7.8 \pm 16.6$ \\
\hline $120^{\circ}$ humeral elevation & $24.2 \pm 12.4$ & $36.8 \pm 12.8$ & $12.6 \pm 15.1$ & $23.2 \pm 19.0$ & $34.8 \pm 17.5$ & $11.6 \pm 20.1$ \\
\hline \multicolumn{7}{|c|}{ Upward/downward rotation $\left({ }^{\circ}\right)$} \\
\hline $0^{\circ}$ humeral elevation & $7.7 \pm 7.6$ & $10.2 \pm 8.2$ & $2.5 \pm 8.5$ & $4.3 \pm 6.5$ & $4.8 \pm 6.6$ & $0.5 \pm 7.4$ \\
\hline $30^{\circ}$ humeral elevation & $9.4 \pm 7.1$ & $13.0 \pm 7.1$ & $2.6 \pm 7.6$ & $6.9 \pm 5.8$ & $8.9 \pm 5.9$ & $2.0 \pm 5.0$ \\
\hline $60^{\circ}$ humeral elevation & $20.1 \pm 6.4$ & $23.6 \pm 6.3$ & $3.5 \pm 6.7$ & $16.6 \pm 7.7$ & $19.1 \pm 6.5$ & $2.5 \pm 6.0$ \\
\hline $90^{\circ}$ humeral elevation & $33.5 \pm 9.1$ & $34.8 \pm 6.5$ & $1.3 \pm 8.9$ & $30.3 \pm 12.0$ & $31.0 \pm 8.2$ & $0.7 \pm 9.0$ \\
\hline $120^{\circ}$ humeral elevation & $34.1 \pm 9.7$ & $36.7 \pm 8.0$ & $2.6 \pm 10.8$ & $33.4 \pm 17.2$ & $38.0 \pm 13.6$ & $4.6 \pm 12.9$ \\
\hline \multicolumn{7}{|c|}{ Anterior/posterior tipping $\left({ }^{\circ}\right)$} \\
\hline $0^{\circ}$ humeral elevation & $7.9 \pm 4.8$ & $11.0 \pm 6.2$ & $3.1 \pm 6.7$ & $10.4 \pm 7.7$ & $12.1 \pm 4.8$ & $1.7 \pm 7.4$ \\
\hline $30^{\circ}$ humeral elevation & $7.2 \pm 5.4$ & $10.4 \pm 6.6$ & $3.2 \pm 7.4$ & $9.6 \pm 8.3$ & $10.8 \pm 5.5$ & $1.2 \pm 7.2$ \\
\hline $60^{\circ}$ humeral elevation & $3.6 \pm 6.6$ & $9.0 \pm 7.8$ & $5.4 \pm 9.0$ & $7.2 \pm 9.9$ & $8.5 \pm 6.7$ & $1.3 \pm 8.5$ \\
\hline $90^{\circ}$ humeral elevation & $-1.2 \pm 8.5$ & $5.0 \pm 9.7$ & $6.2 \pm 11.1$ & $4.0 \pm 9.6$ & $5.7 \pm 6.9$ & $1.7 \pm 9.8$ \\
\hline $120^{\circ}$ humeral elevation & $-0.5 \pm 9.1$ & $5.1 \pm 9.6$ & $5.6 \pm 10.8$ & $3.6 \pm 8.6$ & $4.9 \pm 7.3$ & $1.3 \pm 10.8$ \\
\hline \multicolumn{7}{|l|}{ Protraction/retraction $\left({ }^{\circ}\right)$} \\
\hline $0^{\circ}$ humeral elevation & $35.1 \pm 15.4$ & $23.5 \pm 7.8$ & $-11.6 \pm 19.6$ & $36.2 \pm 17.0$ & $28.7 \pm 8.5$ & $-7.5 \pm 14.0$ \\
\hline $30^{\circ}$ humeral elevation & $35.7 \pm 15.0$ & $24.0 \pm 7.8$ & $-11.7 \pm 19.5$ & $36.8 \pm 17.8$ & $28.5 \pm 8.4$ & $-8.1 \pm 15.7$ \\
\hline $60^{\circ}$ humeral elevation & $37.8 \pm 14.7$ & $26.4 \pm 7.5$ & $-11.4 \pm 19.7$ & $36.1 \pm 20.2$ & $30.5 \pm 8.3$ & $-5.6 \pm 17.4$ \\
\hline $90^{\circ}$ humeral elevation & $40.6 \pm 14.7$ & $30.0 \pm 7.7$ & $-10.6 \pm 20.0$ & $33.2 \pm 17.4$ & $33.5 \pm 8.0$ & $0.3 \pm 14.8$ \\
\hline $120^{\circ}$ humeral elevation & $40.9 \pm 14.9$ & $30.0 \pm 8.2$ & $-10.9 \pm 20.0$ & $33.9 \pm 17.7$ & $35.5 \pm 9.4$ & $1.6 \pm 16.1$ \\
\hline \multicolumn{7}{|l|}{ Elevation/depression $\left({ }^{\circ}\right)$} \\
\hline $0^{\circ}$ humeral elevation & $4.5 \pm 6.1$ & $8.4 \pm 7.5$ & $3.9 \pm 6.6$ & $2.2 \pm 9.1$ & $6.3 \pm 6.1$ & $4.1 \pm 8.4$ \\
\hline $30^{\circ}$ humeral elevation & $5.3 \pm 5.9$ & $9.5 \pm 7.1$ & $4.2 \pm 5.9$ & $4.1 \pm 8.3$ & $7.5 \pm 6.7$ & $3.4 \pm 7.6$ \\
\hline $60^{\circ}$ humeral elevation & $9.8 \pm 5.8$ & $14.7 \pm 6.9$ & $4.9 \pm 5.5$ & $14.7 \pm 9.8$ & $12.3 \pm 7.2$ & $-2.4 \pm 10.5$ \\
\hline $90^{\circ}$ humeral elevation & $15.7 \pm 6.3$ & $21.0 \pm 7.4$ & $5.3 \pm 6.8$ & $23.2 \pm 14.2$ & $18.4 \pm 7.9$ & $-4.8 \pm 15.7$ \\
\hline $120^{\circ}$ humeral elevation & $16.0 \pm 6.5$ & $22.0 \pm 7.7$ & $6.0 \pm 6.5$ & $25.0 \pm 14.0$ & $22.0 \pm 9.5$ & $-3.0 \pm 15.6$ \\
\hline
\end{tabular}


Table 9: Means and standard deviations (SD) of the SANE and DASH rankings before and after the intervention and the change score

\begin{tabular}{ccccccc}
\hline & $\begin{array}{c}\text { Pre } \\
\text { Mean } \pm \text { SD }\end{array}$ & $\begin{array}{c}\text { Intervention } \\
\text { Meant } \pm \text { SD }\end{array}$ & $\begin{array}{c}\text { Change } \\
\text { Mean } \pm \text { SD }\end{array}$ & $\begin{array}{c}\text { Pre } \\
\text { Mean } \pm \text { SD }\end{array}$ & $\begin{array}{c}\text { Control } \\
\text { Meant } \pm \text { SD }\end{array}$ & $\begin{array}{c}\text { Change } \\
\text { Mean } \pm \text { SD }\end{array}$ \\
\hline SANE & $89.6 \pm 16.6$ & $90.3 \pm 10.5$ & $0.7 \pm 15.0$ & $92.0 \pm 9.5$ & $92.1 \pm 8.7$ & $0.1 \pm 8.8$ \\
$\begin{array}{c}\text { DASH } \\
\text { Composite } \\
\text { Score }\end{array}$ & $6.3 \pm 11.8$ & $10.0 \pm 15.6$ & $3.7 \pm 14.7$ & $6.3 \pm 13.4$ & $4.5 \pm 7.4$ & $-1.8 \pm 9.7$ \\
& & & & & &
\end{tabular}




\section{Appendices}

\section{Appendix 1: SANE AND DASH}

SANE Score:

On a scale of 0-100 (with 100 being normal) how would you rate your shoulder today?

Sports Module of the Disabilities of the Arm, Shoulder and Hand:

Please circle the number that best describes your physical ability in the past week. Did you have difficulty:

$\begin{array}{ccccc}\text { No } & \begin{array}{c}\text { Mild } \\ \text { Difficulty }\end{array} & \begin{array}{c}\text { Moderate } \\ \text { Difficulty }\end{array} & \begin{array}{c}\text { Severe } \\ \text { Difficulty }\end{array} & \text { Unable } \\ \text { Difficulty } & & & & \end{array}$

1. using your usual technique for playing your sport?

\section{1}

2

3

4

5

\section{2. playing your sport} because of arm, shoulder or hand 1

2

3

4

5 pain?

3. playing your sport as well as you would like?

1

2

3

4 5

4. spending your usual amount of time practicing or playing 1 2

3

4 5 your sport? 
Appendix 2: Manuscript formatted for Clinical Journal of Sports Medicine

\title{
EFFECT OF A 6-WEEK STRENGTHENING PROGRAM ON SHOULDER AND SCAPULAR STABILIZER STRENGTH AND SCPAULAR KINEATICS IN DIVINSION I COLLEGIATE SWIMMERS
}

\author{
Elizabeth Eve Hibberd, LAT, ATC \\ Graduate Student \\ University of North Carolina at Chapel Hill \\ Chapel Hill, NC \\ Joseph B. Myers PhD, ATC \\ Associate Professor \\ University of North Carolina at Chapel Hill \\ Chapel Hill, NC \\ William Prentice, $\mathrm{PhD}$, ATC \\ Associate Professor \\ University of North Carolina at Chapel Hill \\ Chapel Hill, NC \\ Jeffrey Spang, MD \\ Orthopedic Surgeon \\ University of North Carolina at Chapel Hill \\ Chapel Hill, NC \\ Sakiko Oyama, MS, ATC \\ Research Assistant \\ University of North Carolina at Chapel Hill \\ Chapel Hill, NC
}

\section{Corresponding Author:}

Elizabeth Hibberd, LAT, ATC

CB\#8700 Fetzer

Sports Medicine and Neuromuscular Research Laboratories

University of North Carolina at Chapel Hill

Chapel Hill, NC 25799-8700

Phone \#: 919-962-2728

Fax \#: 919-962-0489

Email: ehibberd@email.unc.edu

\section{Acknowledgements:}

We would like to thank the Theraband Academy for providing the Theraband ${ }^{\circledR}$ rubber tubing that was used in the study. We would also like to thank the UNC Swimming Coaches and Team for volunteering to be subjects in the study. 


\begin{abstract}
Objective: To assess the effectiveness of a 6-week intervention program to address
\end{abstract}

shoulder and scapular muscle strength and scapular kinematics in collegiate swimmers.

Design: Randomized control trial

Setting: University Research Laboratory

Participants: Forty-four Division one collegiate swimmers with current shoulder injuries

Interventions: The intervention program was completed 3 times per week for 6 weeks.

The program included strengthening exercises completed using resistance tubing: Ys, Ts, Ws, Shoulder Flexion, Low Rows, Throwing Acceleration and Deceleration, scapular punches, and Shoulder Internal Rotation and External Rotation at $90^{\circ}$ abduction, and 2 stretching exercises: corner stretch and sleeper stretch.

Main Outcome Measurements: Scapular kinematics, shoulder and scapular muscle strength, and subjective rankings assessed before and after the intervention period.

Results: There were trends towards significance in shoulder flexion and abduction with greater improvement occurring in the intervention group. Scapular kinematic data revealed increased scapular internal rotation, protraction, and elevation in all subjects at post testing, but there was not a significant effect of group on the individual kinematic variables. There was no significant effect of group on subjective rating of the shoulder. Conclusions: The current exercise program was not effective in altering variables, but may serve as a framework for future programs. Modifications to the current program are needed to increase the benefits to collegiate swimmers. These modifications include: adding more stretching exercises, eliminating exercises that overlaps with what is performed in the weight room and dry land training and exercises that target the muscles adequately strengthened from swim training, and timing of implementation. 
Key Words: Injury Prevention, Shoulder Injury, Swimmers Word Count: 250 INTRODUCTION

Competitive swimmers train approximately 10,000-14,000 meters per day, 6-7 times per week, which correlates to 16,000 shoulder revolutions per week (Stocker, Pink et al. 1995; Pink and Tibone 2000). Significant demand is placed on the shoulder, as $90 \%$ of the propulsive force during swimming comes from the upper extremity (Johnson 1988). Because of this, shoulder pain has become commonplace in competitive swimming, comprising at least 55\% of all injuries (McFarland and Wasik 1996). Interfering shoulder pain has been reported in $45-87 \%$ of all swimmers during their careers (Johnson 1988; Beach, Whitney et al. 1992; Stocker, Pink et al. 1995; Urwin, Symmons et al. 1998; Pink and Tibone 2000; Bongers 2001).

The high frequency and intensity of training often leads to "swimmer's shoulder" which is the general term for a shoulder overuse injury in competitive swimmers (Richardson 1986). In swimmers, the biomechanics of the freestyle stroke exacerbate impingement symptoms. While completing the freestyle stroke, $25 \%$ of the stroke occurs in a position that causes subacromial impingement (Yanai and Hay 2000). The repetitive stress placed on the muscles throughout the stroke may lead to shoulder pain and impingement symptoms. In addition to the repetitive nature of the sport, swimmers have a distinct physical profile that predisposes them to shoulder injury and pain. Altered range of motion, muscular imbalances, and forward shoulder posture may predispose swimmers to injuries by altering scapular kinematics and causing abnormal stress on surrounding musculature (Beach, Whitney et al. 1992; Bak and Fauno 1997; Bak and Magnusson 1997; Kebaetse, McClure et al. 1999; Wang, McClure et al. 1999; Finley and 
Lee 2003).

Few studies have evaluated a prevention program designed specifically for swimmers that addresses the known weaknesses and altered movement patterns of these athletes. Therefore, the purpose of this study was to determine the effects of a 6-week intervention program on shoulder strength and scapular kinematics in Division I collegiate swimmers.

\section{MATERIALS AND METHODS}

\section{Subjects}

Forty-four subjects were pre-tested for participation in the current study (Table 1). Seven subjects were excluded due to non-compliance with the intervention program, injury, or failure to report for post-testing. Therefore, thirty-seven subjects were post tested. Scapular kinematics data from six subjects' were lost due to malfunction of the electromagnetic tracking receivers.

Participants were recruited from a Division I swimming team. Participants were included in the study if they participated in swimming for at least 30 minutes per day for 4 days per week, participated in all team weight-lifting sessions, and completed at least 15 of the 18 training sessions. Subjects were excluded from the study if they were diagnosed with a shoulder injury or if they developed pain during the course of the intervention period. All participants read and signed a consent form approved by the university's Institutional Review Board.

\section{Procedures}

All subjects reported for a pretest screening for assessments of strength, scapular 
kinematics, and subjective reporting of shoulder function. Isometric strength was measured using a hand-held dynamometer (HHD) (Lafayette Inc. Lafayette, IN:Model\#01163), which have been shown to be reliable and valid measures for assessing strength of the shoulder and scapular stabilizing muscles (Stratford and Balsor 1994; Leggin, Neuman et al. 1996; Hayes, Walton et al. 2002; McClure, Balaicuis et al. 2007). Strength measurements were taken for shoulder flexion, extension, abduction, adduction, internal and external rotation and scapular retraction, retraction with downward rotation, and retraction with upward rotation. Each position was measured 3 times according to a procedure described by Kendall (Kendall, McCreary et al. 2005).

Scapular kinematic variables were measured using Motion Monitor electromagnetic tracking device (Innovative Sports Training Inc,Chicago,Ill). The electromagnetic tracking device integrated with the Motion Monitor software used the data acquired through electromagnetic receivers for the calculation of receiver position and orientation relative to the transmitter. The receivers were placed on the spinous process of $\mathrm{C} 7$, acromion process and mid-shaft of the posterior humerus on the dominant arm with one attached to the stylus to digitize the anatomical landmarks. Validity of the instrument for the assessment of scapular kinematics has been established previously (Karduna, McClure et al. 2001). Subjects performed a humeral elevation task in the scapular plane, which is defined as $30^{\circ}$ anterior to the frontal plane. The subjects performed 15 elevations at a rate of 4 seconds per repetition (Karduna, McClure et al. 2000; McClure, Bialker et al. 2004; Thigpen, Padua et al. 2006). Kinematics were sampled at 100Hz (Karduna, McClure et al. 2001; Myers, Laudner et al. 2005; Thigpen, Gross et al. 2005). 
The Single Assessment Numeric Evaluation (SANE) and sports module from the Disabilities of the Arm, Shoulder, and Hand (DASH) were used to assess subjective measure of shoulder functioning. Both SANE and DASH have been found to be a reliable and valid assessment tool (Williams, Gangel et al. 1999; Williams, Taylor et al. 2000; Huisstede, Feleus et al. 2009).

Following pretest screening, the subjects were randomly assigned into an intervention and control group after being stratified by sex. During the 6-week intervention period, participants in the intervention group performed the exercise program after practice 3 times/week in addition to the regular training. The principal investigator monitored training sessions to provide feedback and tracked compliance. The intervention program included 2 sets of 15 repetitions of the following strengthening exercises: shoulder flexion, shoulder external and internal rotation at $90^{\circ}$ abduction, low rows, D2 pattern acceleration and deceleration, scapular punches, Ys, Ts and Ws. These exercises have been shown to be the most effective resistance tubing exercises for activating weak muscles in swimmers (Figure 1)(Moseley, Jobe et al. 1992; Cordasco, Wolfe et al. 1996; Hintermeister, Lange et al. 1998; Decker, Hintermeister et al. 1999; Ekstrom, Donatelli et al. 2003; Myers, Pasquale et al. 2005; Cools, Dewitte et al. 2007; Reinold, Macrina et al. 2007). Subjects performed 2 sets of 30 seconds of stretching exercises: sleeper stretch and corner stretch, which have been shown to be effective in improving range of motion and allowing optimal functioning of the shoulder musculature (Figure 2)(Borstad and Ludewig 2006; McClure, Balaicuis et al. 2007).

At the first session, all subjects were given resistance tubing (Thera-band ${ }^{\circledR}$, Hygenic Corp, Akron, Ohio) to determine the band with appropriate resistance. The 
subjects performed 5 repetitions of each exercise with different levels of resistance tubing in order to estimate which resistance was appropriate (Kluemper, Uhl et al. 2006). Subjects were instructed to choose a level of resistance that was challenging, but that the exercises could be completed. Feedback from the subject and observation of proper form was used to determine if the appropriate resistance level had been chosen. Subjects were re-evaluated every 2 weeks to determine if they needed to change the resistance level that was being used. Strength and scapular kinematics were re-assessed following the 6-week intervention period. Researchers performing the measurements were blinded to the group assignment to prevent bias.

\section{Data Reduction}

Strength data were normalized to body mass and calculated as a three-trial mean for each strength variable. Raw scapular kinematic data were filtered with a low pass $10 \mathrm{~Hz}$ Butterworth filter. Receiver position and orientation data of the thoracic, scapular, and humeral receivers were transformed into a local coordinate system for each of the respective segments, based on recommendations from the International Society of Biomechanics (Wu, van der Helm et al. 2005). Orientation of the scapula was determined as rotation about the $y$-axis (internal/external rotation), rotation about the z-axis (upward/downward rotation) and rotation about the x-axis (anterior/posterior tipping). Euler angles ( $\mathrm{Y}-\mathrm{X}-\mathrm{Z}$ order) were used to determine the scapular orientation with respect to the thorax and Euler angles (Y-X-Y order) were used to determine the position of the humerus relative to the thorax ( $\mathrm{Wu}$, van der Helm et al. 2005). Based on ISB recommendations scapular movements in internal rotation, downward rotation and posterior tilt directions were indicated by the positive numbers (Wu, van der Helm et al. 
2005). For ease of interpretation, scapular upward rotation values were multiplied by -1 to make upward rotation a positive movement. Scapular kinematic variables at $0^{\circ}, 30^{\circ}$, $60^{\circ}, 90^{\circ}$, and $120^{\circ}$ of humeral elevation were calculated as means of middle five repetitions. A composite score for the Sports Module of the Disabilities of the Arm, Shoulder, and Hand was calculated based on the subject's responses (Solway, Beaton et al. 2002).

\section{Statistical Analysis}

Two-way ANOVAs with one within-factor (session) and one between-factor (group) were run to determine differences in normalized strengths and SANE and DASH rankings between groups and sessions. Bonferroni post-hoc analyses were conducted to determine where the significant interactions or main effects existed. Three-way ANOVAs with two within-factors (session and angle) and one-between factor (group) were used to examine the interactions and main effects for scapular kinematic variables. Huynh-Feldt correction was used whenever the assumption of sphericity was rejected. Bonferroni post hoc analyses were conducted to determine where the differences occurred. An a priori alpha levels was set at .05. All analyses were run using SPSS version 16.0 for Macintosh.

\section{Results}

The shoulder strength data are presented in Table 2 and Figures 3-4. There was a significant group by session interaction in flexion $\left(\mathrm{F}_{(1,35)}=5.972, \mathrm{p}=0.020\right)$ and abduction $\left(F_{(1,35)}=6.635, p=0.014\right)$ strength. Bonferroni post-hoc analyses were not significant for shoulder flexion and abduction strength pre-intervention to post-intervention in either group or between groups at either test session. Group by session interactions were insignificant for other strength variables. There was a significant main effect of session 
on extension strength $\left(\mathrm{F}_{(1,35)}=8.783, \mathrm{p}=0.005\right)$ and scapular retraction $\left(\mathrm{F}_{(1,35)}=55.212, \mathrm{p}<0.005\right)$ when the data were collapsed across groups. On average, subjects increased their shoulder extension strength by $4.16 \%$ and scapular retraction strength by $6.25 \%$ between sessions. No other session or group main effects were present.

Scapular kinematic data are presented in Table 3. Angle by group by session three-way interactions were insignificant for all scapular kinematic variables. There was a significant angle by group interaction on internal/external rotation $\left(\mathrm{F}_{(4,108)}=5.453, \mathrm{p}=0.018\right)$. Bonferroni post-hoc analysis demonstrated that the scapula was more internally rotated in the treatment group participants compared to the control group participants at humeral elevation angle of $0^{\circ}\left(\mathrm{t}_{(58)}=2.918, \mathrm{p}=0.005\right)$ and $30^{\circ}$ $\left(\mathrm{t}_{(60)}=2.840, \mathrm{p}=0.006\right)$, but not at humeral elevation angles of $60^{\circ}, 90^{\circ}$, and $120^{\circ}$ when the data were collapsed across sessions. There was a significant angle by group interaction for scapular elevation/depression angles $\left(\mathrm{F}_{(4,100)}=4.320, \mathrm{p}=0.038\right)$, but post-hoc analysis did not reveal between-session differences at any humeral elevation angle. There were no significant angle by group interactions in upward/downward rotation, anterior/posterior tilt, protraction/retraction. There were no significant angle by session or angle by group interactions of the scapular kinematic variables.

A significant main effect of session was present for internal/external rotation $\left(\mathrm{F}_{(1,27)}=25.085, \mathrm{p}<0.0005\right)$, protraction/retraction $\left(\mathrm{F}_{(1,25)}=10.88, \mathrm{p}=0.003\right)$, and elevation/depression $\left(\mathrm{F}_{(1,25)}=4.279, \mathrm{p}=0.049\right)$. On average, the swimmers' scapulae were $11.1^{\circ} \pm 2.21^{\circ}$ more internally rotated, $8.83^{\circ} \pm 2.67^{\circ}$ more protracted and $2.85^{\circ} \pm 1.38^{\circ}$ more elevated at the post-intervention session when average over groups and angles. 
The SANE and DASH subjective ranking data are presented in Table 4. There was no significant group by session interaction or main effects for SANE or DASH composite score.

\section{Discussion}

While not statistically significant, trends were found indicating intervention group subjects having stronger flexion and abduction strength compared to the subjects in the control group. These trends resulted from a modest strength gain in the intervention group and a small strength loss in the control group. Continuation of the exercise program may result in clinically significant strength gain.

In addition, a non-significant trend was found that the adduction:abduction strength ratio in the control group increased from 1.37 to 1.60 , while the ratio in the intervention group decreased from 1.35 to 1.29 . Greater adduction to abduction strength ratios may be caused by overdevelopment of the pectoralis major and latissimus dorsi due to the demands of the sport, which can lead to injury by creating forward shoulder posture (Pink, Perry et al. 1991). The trend in this study may indicate that the intervention program had an effect in negating the change in strength ratio occurring with swim training, and therefore may have a protective effect on the development of shoulder injuries.

The program did not result in improvements of the shoulder adduction, extension, internal rotation and external rotation strength or the scapular stabilizer strengths. Shoulder adduction, extension, and internal rotation are the primary movements required to propel the body through the water and external rotation is the primary motion during the recovery phase (Yanai, Hay et al. 2000). With the amount of yardage that the 
swimmers were completing and the concurrent demands on these muscle groups, the resistance tubing most likely did not provide enough resistance to create strength improvements.

To date, only a few studies have investigated the effectiveness of the intervention program to improve shoulder strength specifically in swimmers. Results from this study were similar to those from a study in collegiate swimmers by Swanik et al (Swanik, Swanik et al. 2002) that found no significant strength differences between control and intervention groups after a 6-week intervention program using resistance tubing and attributed their lack of findings to preseason conditioning. Wang et al (Wang, McClure et al. 1999) introduced a 6-week intervention program in subjects with impingement that found a significant increase external and internal rotation and horizontal abduction strength. McClure et al (McClure, Bialker et al. 2004) found increase abduction and internal rotation strength in subjects with impingement following an intervention program. The results of the Wang and McClure study may differ from the current study because subjects in their studies were experiencing impingement symptoms at the time of testing and were not competitive athletes.

The subjects in the intervention group had significantly greater scapular internal rotation compared to the control group at $0^{\circ}$ and $30^{\circ}$ of humeral elevation. Since the subjects were randomly assigned to the groups, it is unknown why the difference was found. However, since the shoulder pain in overhead athletes is commonly experienced at higher humeral elevation angle, this difference is likely not related to swimmer's vulnerability to shoulder impingement. 
Interestingly, this study found that swimmers' scapulae became more internally rotated, protracted, and elevated at the post-intervention screening compared to the preintervention screening regardless of the group assignment. The changes in scapular kinematics may be attributed to increased tightness of the posterior shoulder and pectoralis muscles that developed in response to increasing training intensity. Subjects with posterior shoulder tightness and and/or tight pectoralis muscles have increased anterior tilt, internal rotation, and downward rotation (Borstad and Ludewig 2005; Borich, Bright et al. 2006). Therefore, muscle imbalances and tightness that develops due to the increased swim training may be responsible for the increased protraction and internal rotation at the post testing.

The findings from this study are consistent with the study by McClure at el (McClure, Bialker et al. 2004) in that the intervention program did not have any effect on scapular kinematics. On the contrary, a study by Wang et al (Wang, McClure et al. 1999) observed a decreased upward rotation and elevation and an increased internal rotation following an exercise program in asymptomatic participants with forward shoulder posture. However, the change in scapular kinematics may be attributed to the fact that the all participants in the study had forward head posture associated with tightness of the pectoralis major and minor muscles.

The current study did not demonstrate statistically significant improvement in DASH or SANE scores from the intervention program. The SANE score has been shown to be a reliable and valid measure of shoulder functioning in the general population, but has not been used in the athletic population (Lysholm and Gillquist 1982; Tegner and Lysholm 1985; Williams, Taylor et al. 2000). The SANE may only reflect how the 
individual's shoulder felt at the time of testing, and not the individual's general perception of their shoulder function. Interestingly there was a trend towards participants in the intervention group having higher DASH score indicating more difficulty performing sport activities, compared to the participants in the control group at the post test. This trend is contrary to the finding from a study by Swanik et al (Swanik, Swanik et al. 2002) that found a decreased incidence of reported shoulder pain in collegiate swimmers after completing a 6-week intervention program. McClure et al (McClure, Bialker et al. 2004) reported improvement in University of Pennsylvania shoulder scale score following a 6-week intervention in subjects with impingement syndrome. In the current study, the subjects who were completing the intervention program often reported being sore after completing the strengthening program. The soreness reported in the intervention program may have caused the subjects to report increased symptoms. Although not ideal to have increased symptoms, this soreness may be necessary in order to achieve strength gain.

Based on the findings of the current study, the intervention program was not successful in improving the variables as hypothesized and would not be an effective program to implement in competitive swimmers. However, the results of the current study did provide valuable framework for how the intervention program could be modified to benefit the competitive swimmer. The intervention program was not robust enough to counteract the effects of swimming on muscle tightness development, as all subjects moved into greater internal rotation, protraction, and elevation. A greater focus on stretching of the anterior shoulder musculature and posterior capsule to reverse the increasing scapular internal rotation and protraction, as well as the upper trapezius to 
reverse the increasing scapular elevation may be needed. Coordination with swimming and strength coaches is needed to prevent overlap in exercises performed, to prevent overtraining and improve compliance to the program.

Finally, the timing and length of the program may be important when introducing an intervention program. Fatigue, muscle soreness, and overtraining are all very common in swimmers during the pre-season training, which may overshadow the positive effects of the intervention program. A more robust effect may be produced if implemented during spring training, when the focus is more on technique than yardage. Continuing the intervention program throughout the season may result in greater improvements to prevent injury.

Swimmers have been taught that shoulder pain is normal and is often unreported. It is possible that subjects who were experiencing shoulder pain throughout the intervention period or at post-test were included in the study due to lack of reporting. Another limitation of this study was that individual effort could not be assessed.

Although the exercise program was explained to participants and a bi-weekly evaluation of technique was performed, some participants may have chosen resistive rubber tubing that was too easy. Data collection and implementation of the strengthening program occurred during the intense training period when the swimmers swim high yardage to build cardiovascular endurance, which may have affected the results. Overtraining and the intensity of the swim conditioning may have masked the effects of the intervention program. 
The physical characteristics and sport specific demands on swimmers are different than any other sport and therefore implementation of an evidence based exercise program tailored to the sport specific needs may decrease the stress on the shoulder and prevent the development of shoulder pain. Additionally, a long-term prospective study assessing the effectiveness of the intervention program in reducing the risk of shoulder injury is needed in order to truly determine how effective a strengthening program is in preventing a shoulder injury. Finally, research examining shoulder injuries and prevention programs in swimmers of all ages is necessary. These swimmers rarely participate in weight lifting or dry-land exercises. Implementing an intervention program in youth swimmers may have a larger impact on these developing muscles and prevent shoulder pain and injuries to promote physical characteristics that prevent shoulder injuries from developing.

\section{CONCLUSION}

The results of the current study did not find significant changes in shoulder or scapular stabilizer strength, scapular kinematics, or subjective shoulder rankings between groups. In addition, it was found that all subjects moved into increased scapular internal rotation, protraction, and elevation due to the demands of increased swim conditioning. Further research is needed to develop intervention programs in swimmers and its longterm effect on injury prevention.

\section{Word Count: 2997}




\section{REFERENCES}

1. Bak K, Fauno P. Clinical findings in competitive swimmers with shoulder pain. Am J Sports Med. Mar-Apr 1997;25(2):254-260.

2. Bak K, Magnusson SP. Shoulder strength and range of motion in symptomatic and pain-free elite swimmers. Am J Sports Med. Jul-Aug 1997;25(4):454-459.

3. Beach ML, Whitney SL, Dickoff-Hoffman S. Relationship of shoulder flexibility, strength, and endurance to shoulder pain in competitive swimmers. J Orthop Sports Phys Ther. 1992;16(6):262-268.

4. Bongers PM. The cost of shoulder pain at work. BMJ. 2001;322(7278):64-65.

5. Borich MR, Bright JM, Lorello DJ, Cieminski CJ, Buisman T, Ludewig PM. Scapular angular positioning at end range internal rotation in cases of glenohumeral internal rotation deficit. J Orthop Sports Phys Ther. Dec 2006;36(12):926-934.

6. Borstad JD, Ludewig PM. The effect of long versus short pectoralis minor resting length on scapular kinematics in healthy individuals. J Orthop Sports Phys Ther. Apr 2005;35(4):227-238.

7. Borstad JD, Ludewig PM. Comparison of three stretches for the pectoralis minor muscle. J Shoulder Elbow Surg. May-Jun 2006;15(3):324-330. 
8. Cools AM, Dewitte V, Lanszweert F, et al. Rehabilitation of scapular muscle balance: which exercises to prescribe? Am J Sports Med. Oct 2007;35(10):17441751.

9. Cordasco FA, Wolfe IN, Wootten ME, Bigliani LU. An electromyographic analysis of the shoulder during a medicine ball rehabilitation program. $A m J$ Sports Med. May-Jun 1996;24(3):386-392.

10. Decker MJ, Hintermeister RA, Faber KJ, Hawkins RJ. Serratus anterior muscle activity during selected rehabilitation exercises. Am J Sports Med. Nov-Dec 1999;27(6):784-791.

11. Ekstrom RA, Donatelli RA, Soderberg GL. Surface electromyographic analysis of exercises for the trapezius and serratus anterior muscles. J Orthop Sports Phys Ther. May 2003;33(5):247-258.

12. Finley MA, Lee RY. Effect of sitting posture on 3-dimensional scapular kinematics measured by skin-mounted electromagnetic tracking sensors. Arch Phys Med Rehabil. Apr 2003;84(4):563-568.

13. Hayes K, Walton JR, Szomor ZL, Murrell GA. Reliability of 3 methods for assessing shoulder strength. $J$ Shoulder Elbow Surg. Jan-Feb 2002;11(1):33-39.

14. Hintermeister RA, Lange GW, Schultheis JM, Bey MJ, Hawkins RJ. Electromyographic activity and applied load during shoulder rehabilitation exercises using elastic resistance. Am J Sports Med. Mar-Apr 1998;26(2):210220. 
15. Huisstede BM, Feleus A, Bierma-Zeinstra SM, Verhaar JA, Koes BW. Is the disability of arm, shoulder, and hand questionnaire (DASH) also valid and responsive. Spine. Feb 15 2009;34(4):E130-138.

16. Johnson D. In Swimming, shoulder the burden. Sportcare Fitness. 1988;MayJune:24-30.

17. Karduna AR, McClure PW, Michener LA. Scapular Kinematics: Effects of altering the Euler Angle of sequence of rotations. J Biomech. 2000;33:1063-1068.

18. Karduna AR, McClure PW, Michener LA, Sennett B. Dynamic measurements of three-dimensional scapular kinematics: a validation study. J Biomech Eng. Apr 2001;123(2):184-190.

19. Kebaetse M, McClure P, Pratt NA. Thoracic position effect on shoulder range of motion, strength, and three-dimensional scapular kinematics. Arch Phys Med Rehabil. Aug 1999;80(8):945-950.

20. Kendall FP, McCreary EK, Provance PG, Rodgers MM, Romani WA. Muscle Testing and Function, with Posture and Pain. Fifth ed. Baltimore: Lippincott Williams \& Wilkins; 2005.

21. Kluemper M, Uhl TL, Hazelrigg H. Effect of Stretching and Strengthening Shoulder Muscles on Forward Shoulder Posture in Competitive Swimmers. $J$ Sport Rehabil. 2006;15:58-70. 
22. Leggin BG, Neuman RM, Iannotti JP, Williams GR, Thompson EC. Intrarater and interrater reliability of three isometric dynamometers in assessing shoulder strength. J Shoulder Elbow Surg. Jan-Feb 1996;5(1):18-24.

23. Lysholm J, Gillquist J. Evaluation of knee ligament surgery results with special emphasis on use of a scoring scale. Am J Sports Med. May-Jun 1982;10(3):150154.

24. McClure P, Balaicuis J, Heiland D, Broersma ME, Thorndike CK, Wood A. A randomized controlled comparison of stretching procedures for posterior shoulder tightness. J Orthop Sports Phys Ther. Mar 2007;37(3):108-114.

25. McClure PW, Bialker J, Neff N, Williams G, Karduna A. Shoulder function and 3-dimensional kinematics in people with shoulder impingement syndrome before and after a 6-week exercise program. Phys Ther. Sep 2004;84(9):832-848.

26. McFarland EG, Wasik M. Injuries in female collegiate swimmers due to swimming and cross training. Clin J Sport Med. Jul 1996;6(3):178-182.

27. Moseley JB, Jr., Jobe FW, Pink M, Perry J, Tibone J. EMG analysis of the scapular muscles during a shoulder rehabilitation program. Am J Sports Med. Mar-Apr 1992;20(2):128-134.

28. Myers JB, Laudner KG, Pasquale MR, Bradley JP, Lephart SM. Scapular position and orientation in throwing athletes. Am J Sports Med. Feb 2005;33(2):263-271. 
29. Myers JB, Pasquale MR, Laudner KG, Sell TC, Bradley JP, Lephart SM. On-theField Resistance-Tubing Exercises for Throwers: An Electromyographic Analysis. J Athl Train. Mar 2005;40(1):15-22.

30. Pink M, Perry J, Browne A, Scovazzo ML, Kerrigan J. The normal shoulder during freestyle swimming. An electromyographic and cinematographic analysis of twelve muscles. Am J Sports Med. Nov-Dec 1991;19(6):569-576.

31. Pink MM, Tibone JE. The painful shoulder in the swimming athlete. Orthop Clin North Am. Apr 2000;31(2):247-261.

32. Reinold MM, Macrina LC, Wilk KE, et al. Electromyographic analysis of the supraspinatus and deltoid muscles during 3 common rehabilitation exercises. $J$ Athl Train. Oct-Dec 2007;42(4):464-469.

33. Richardson AR. The biomechanics of swimming: the shoulder and knee. Clin Sports Med. Jan 1986;5(1):103-113.

34. Solway S, Beaton D, McConnel S, Bombardier C. The DASH Outcome Measure User's Manual. 2nd ed. Toronto: Institute for Work \& Health; 2002.

35. Stocker D, Pink M, Jobe FW. Comparison of shoulder injury in collegiate- and master's-level swimmers. Clin J Sport Med. 1995;5(1):4-8.

36. Stratford PW, Balsor BE. A comparison of make and break tests using a handheld dynamometer and the Kin-Com. J Orthop Sports Phys Ther. Jan $1994 ; 19(1): 28-32$. 
37. Swanik K, Swanik C, Lephart SM, Huxel K. The effect of functional training on the incidence of shoulder pain and strength in intercollegiate swimmers. J Sport Rehabil. 2002;11(2):142-154.

38. Tegner Y, Lysholm J. Rating systems in the evaluation of knee ligament injuries. Clin Orthop Relat Res. Sep 1985(198):43-49.

39. Thigpen CA, Gross MT, Karas SG, Garrett WE, Yu B. The repeatability of scapular rotations across three planes of humeral elevation. Res Sports Med. JulSep 2005;13(3):181-198.

40. Thigpen CA, Padua DA, Morgan N, Kreps C, Karas SG. Scapular kinematics during supraspinatus rehabilitation exercise: a comparison of full-can versus empty-can techniques. Am J Sports Med. Apr 2006;34(4):644-652.

41. Urwin M, Symmons D, al e. Estimating the burden of muscuolskeletal disorders in the community: The comparative prevalence of symptoms at different antatomical sites and the relation to social deprivation. Ann Rheum Dis. 1998;57(11):649-655.

42. Wang $\mathrm{CH}$, McClure $\mathrm{P}$, Pratt NE, Nobilini R. Stretching and strengthening exercises: their effect on three-dimensional scapular kinematics. Arch Phys Med Rehabil. Aug 1999;80(8):923-929.

43. Williams GN, Gangel TJ, Arciero RA, Uhorchak JM, Taylor DC. Comparison of the Single Assessment Numeric Evaluation method and two shoulder rating 
scales. Outcomes measures after shoulder surgery. Am J Sports Med. Mar-Apr 1999;27(2):214-221.

44. Williams GN, Taylor DC, Gangel TJ, Uhorchak JM, Arciero RA. Comparison of the single assessment numeric evaluation method and the Lysholm score. Clin Orthop Relat Res. Apr 2000(373):184-192.

45. Wu G, van der Helm FC, Veeger HE, et al. ISB recommendation on definitions of joint coordinate systems of various joints for the reporting of human joint motion-Part II: shoulder, elbow, wrist and hand. J Biomech. May 2005;38(5):981-992.

46. Yanai T, Hay JG. Shoulder impingement in front-crawl swimming: II. Analysis of stroking technique. Med Sci Sports Exerc. Jan 2000;32(1):30-40.

47. Yanai T, Hay JG, Miller GF. Shoulder impingement in front-crawl swimming: I. A method to identify impingement. Med Sci Sports Exerc. Jan 2000;32(1):21-29. 


\section{Figure Legend}

Figure 1: Shoulder and Scapular Stabilizing Strengthening exercises included in the intervention program

Figure 2: Stretching exercises included in the intervention program

Figure 3: Shoulder flexion strength changes between sessions by group

Figure 4: Shoulder abduction strength changes between sessions by group

Table 1: Subject demographics

Table 2: Means and standard deviations (SD) of the shoulder and scapular stabilizer strength (\% body mass $(\mathrm{BM}))$ before and after the intervention and the change score

Table 3: Means and standard deviations (SD) of the Scapular Kinematics during humeral elevation task before and after the intervention and the change score

Table 4: Means and standard deviations (SD) of the SANE and DASH rankings before and after the intervention and the change score 
Figure 1

a. Shoulder Flexion
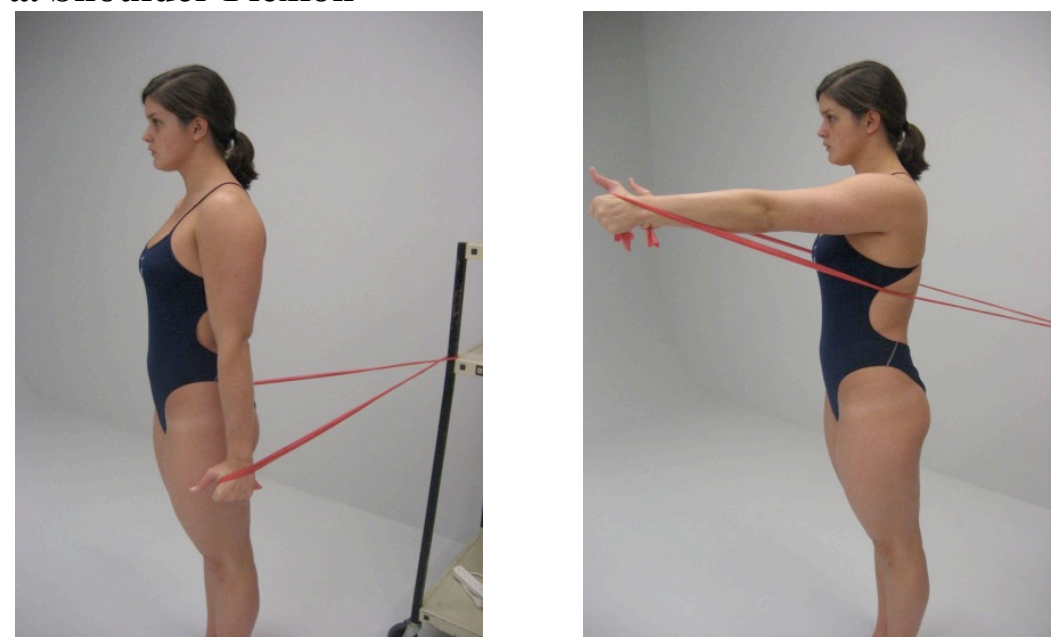

\section{b. Shoulder Extension}
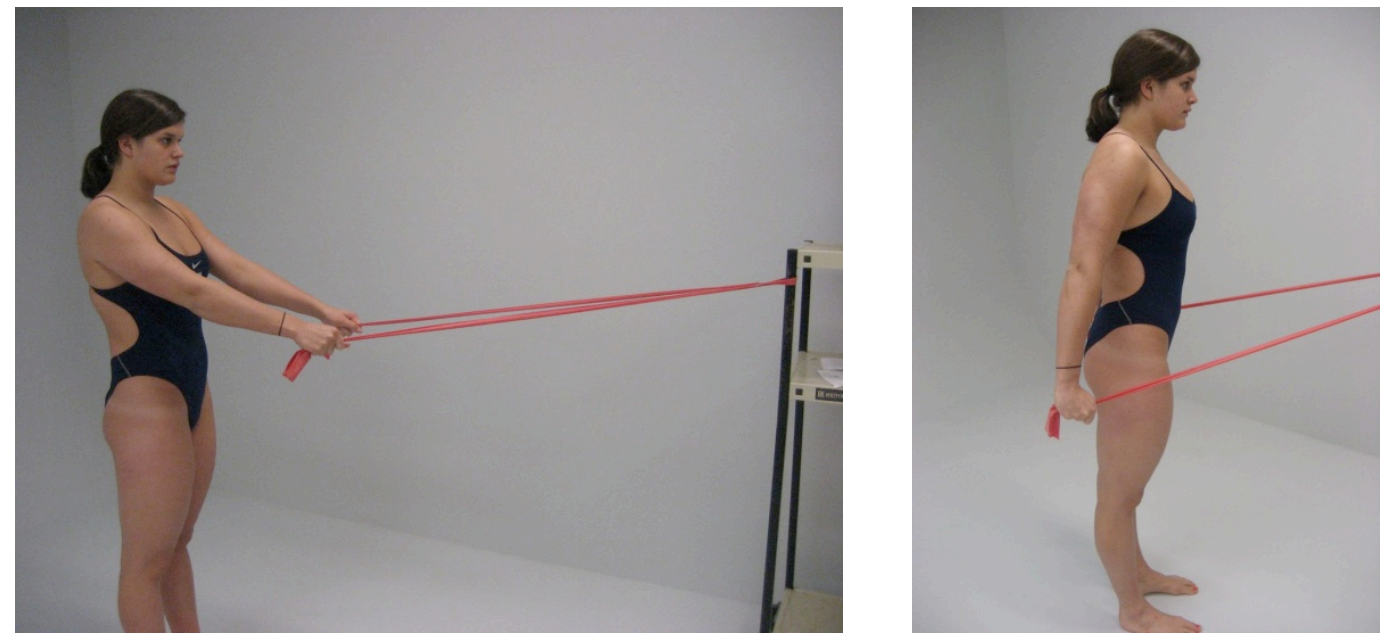

c. Shoulder ER at $90^{\circ}$
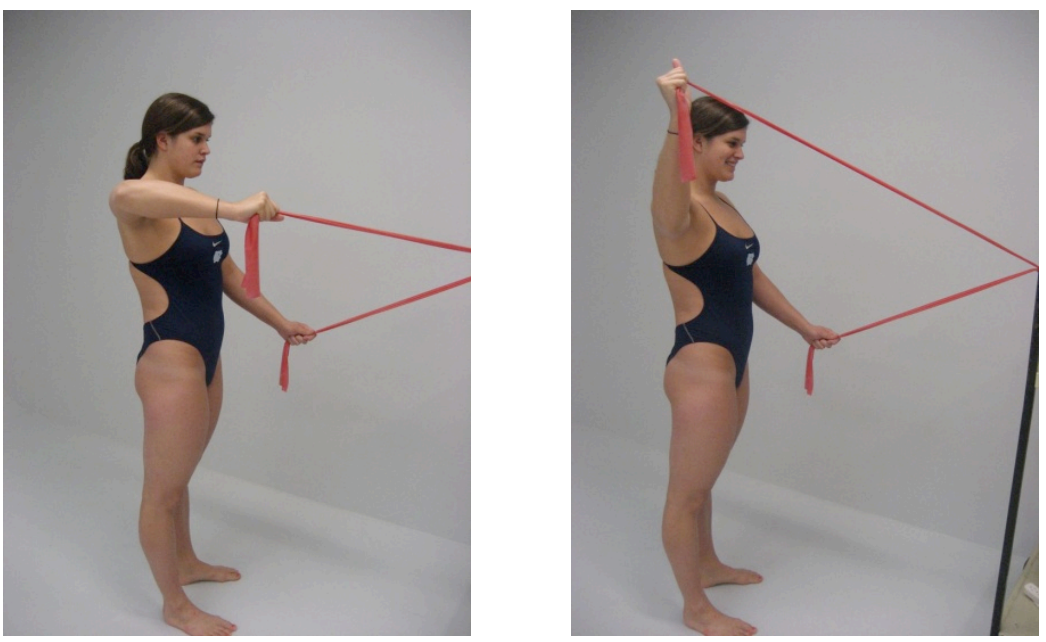
d. Shoulder IR at $90^{\circ}$
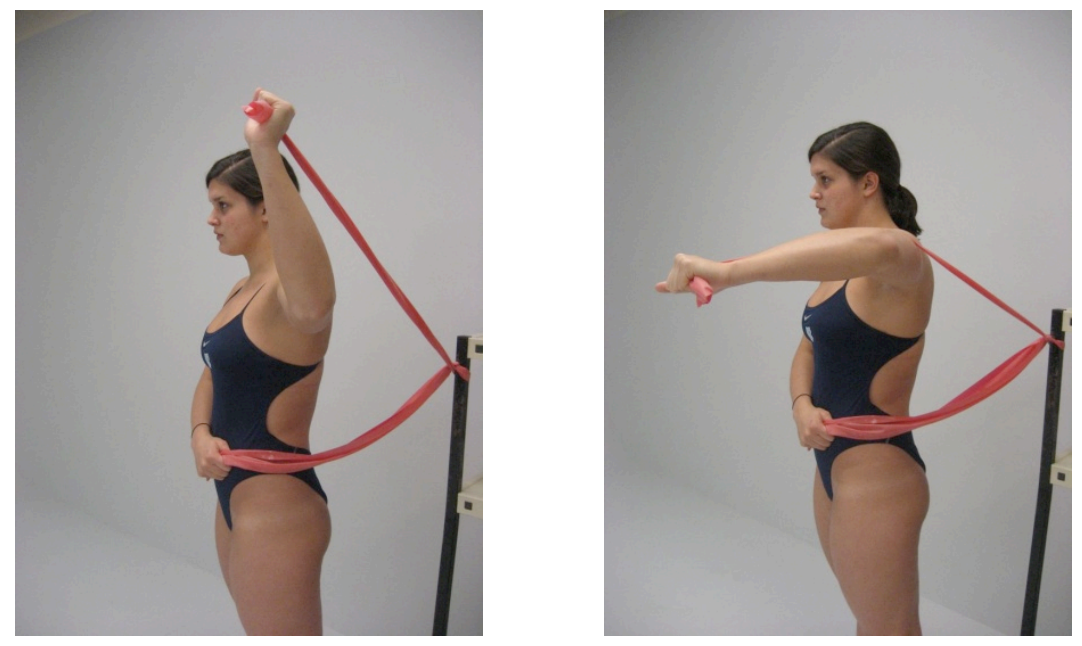

\section{e. Low Rows}
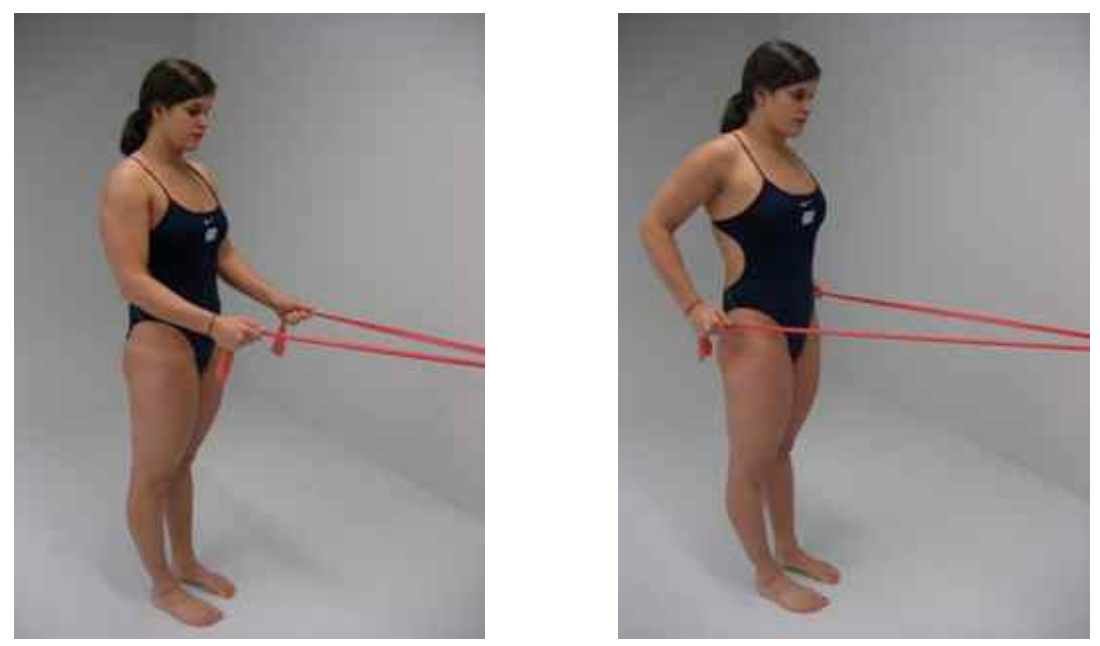

\section{f. Throwing Acceleration}
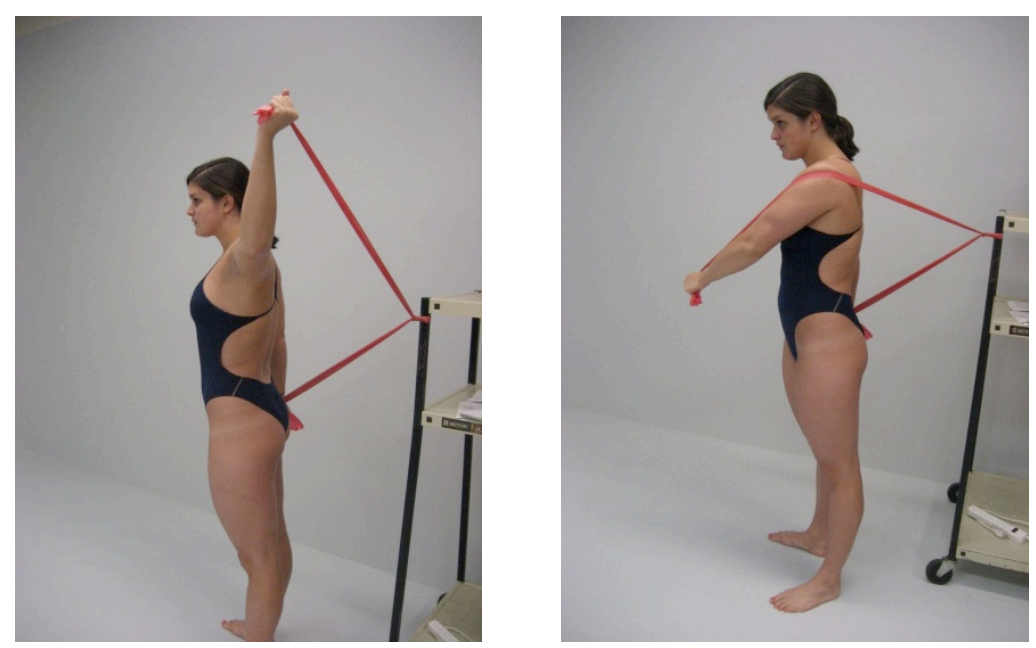


\section{g. Throwing Deceleration}

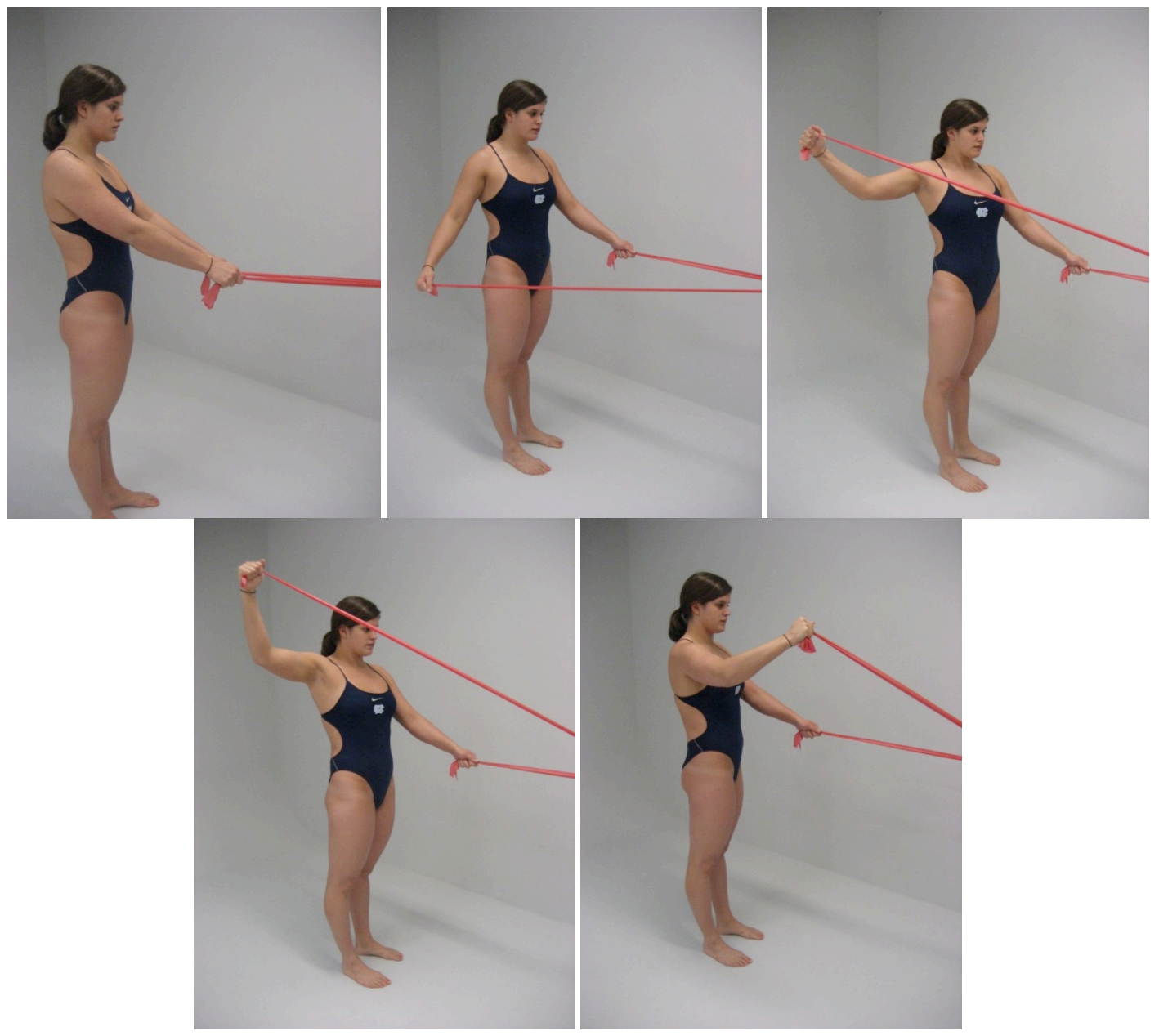

h. Ys
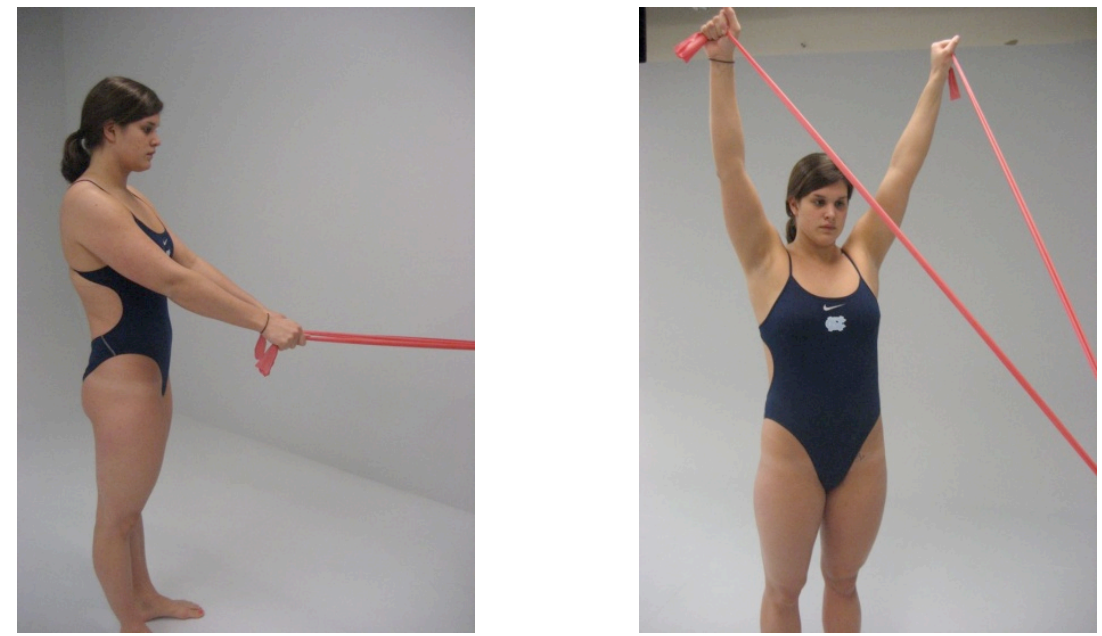
i. Ts

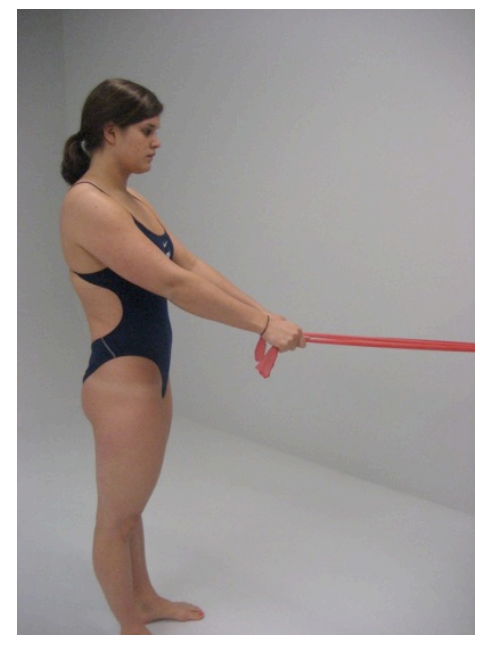

j: Ws

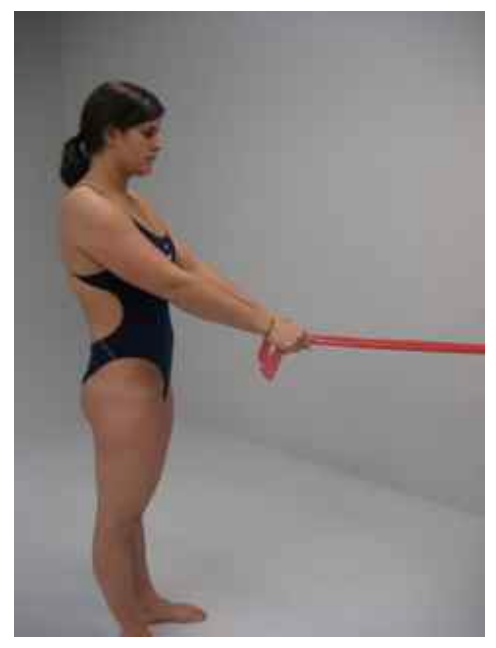

\section{k: Scapular Punches}

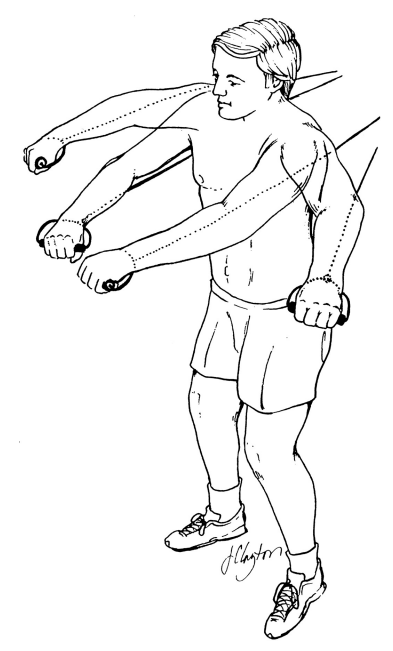

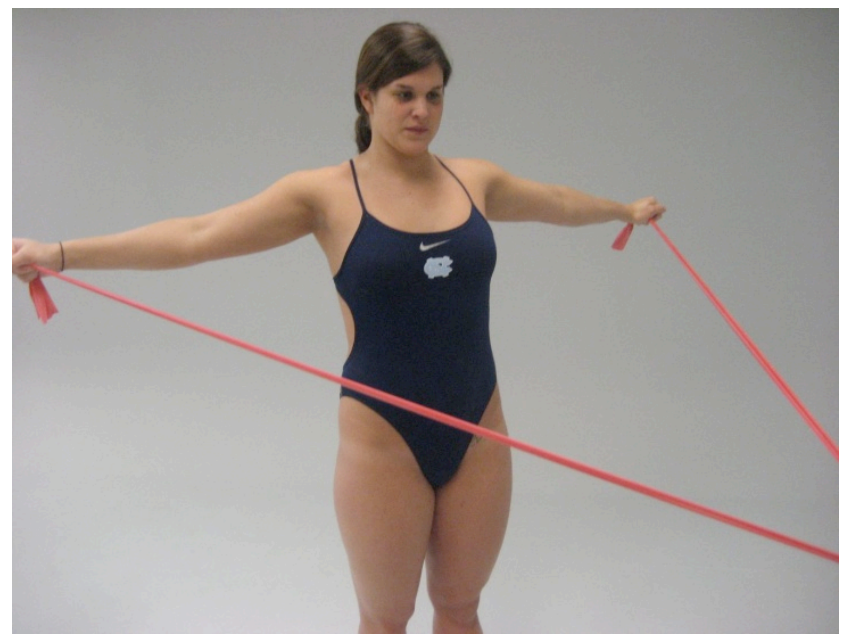

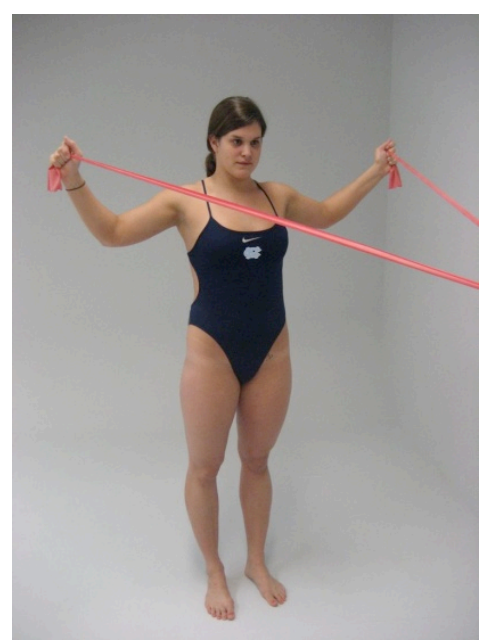


Figure 2

a. Sleeper Stretch

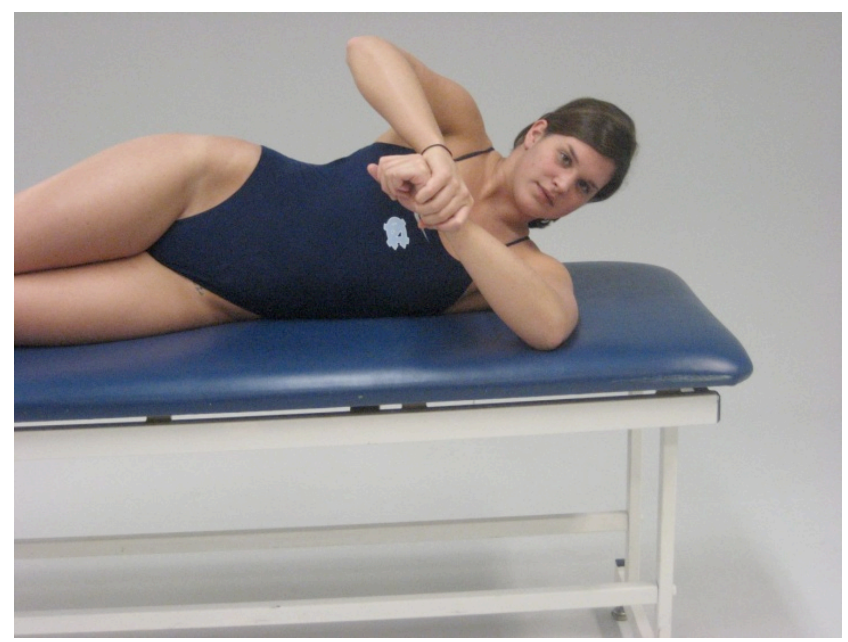

b: Corner Stretch

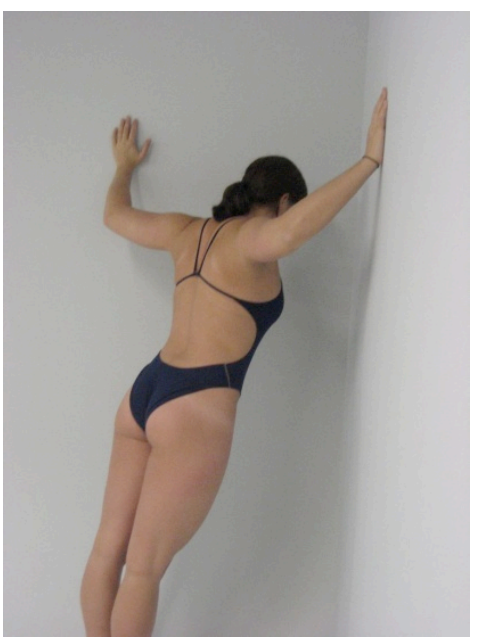


Figure 3

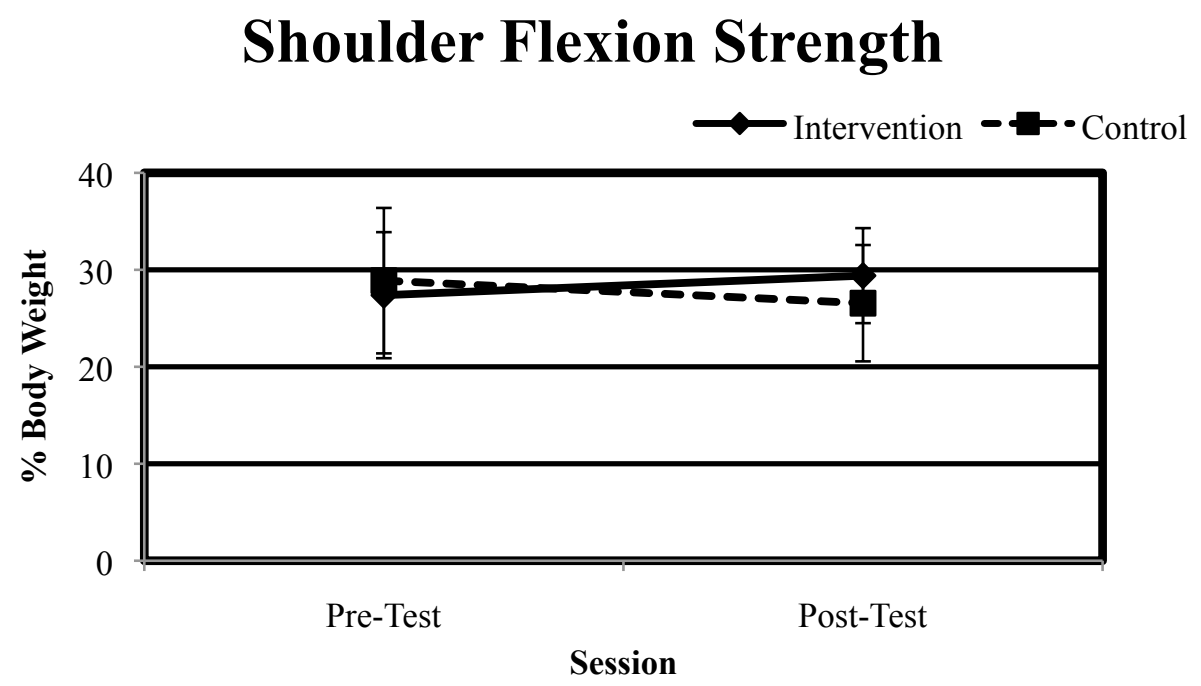


Figure 4

\section{Shoulder Abduction Strength}

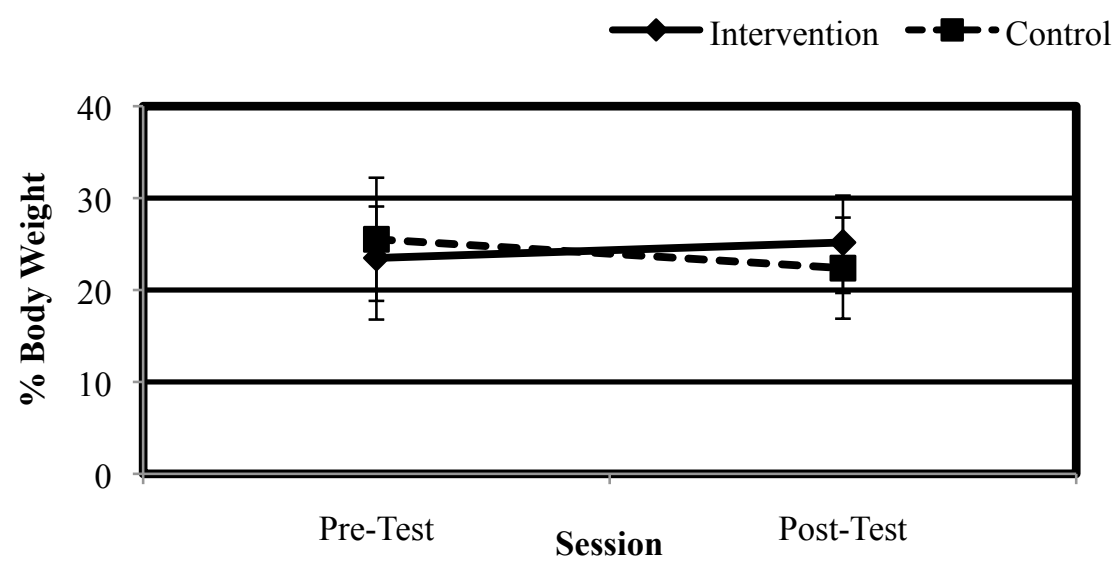


Table 1

\begin{tabular}{ccc}
\hline & Intervention & Control \\
\hline Number of Subjects (n) & 20 & 17 \\
Males / Females & $10 / 10$ & $8 / 9$ \\
Age (yrs) & $19.2 \pm 1.2$ & $19.4 \pm 1.2$ \\
Mass (kg) & $73.1 \pm 9.9$ & $72.8 \pm 12.4$ \\
Height (cm) & $177.5 \pm 9.8$ & $178.1 \pm 8.7$ \\
\hline
\end{tabular}


Table 2

\begin{tabular}{|c|c|c|c|c|c|c|}
\hline & \multicolumn{3}{|c|}{ Intervention } & \multicolumn{3}{|c|}{ Control } \\
\hline & $\begin{array}{c}\text { Pre } \\
\text { Mean } \pm \text { SD }\end{array}$ & $\begin{array}{c}\text { Post } \\
\text { Mean } \pm \text { SD }\end{array}$ & $\begin{array}{c}\text { Change } \\
\text { Mean } \pm \text { SD }\end{array}$ & $\begin{array}{c}\text { Pre } \\
\text { Mean } \pm \text { SD }\end{array}$ & $\begin{array}{c}\text { Post } \\
\text { Mean } \pm \text { SD }\end{array}$ & $\begin{array}{c}\text { Change } \\
\text { Mean } \pm \text { SD }\end{array}$ \\
\hline Flexion (\%BM) & $27.4 \pm 6.5$ & $29.4 \pm 4.9$ & $2.0 \pm 5.0$ & $28.9 \pm 7.5$ & $26.6 \pm 6.0$ & $-2.3 \pm 5.8$ \\
\hline Extension (\%BM) & $25.2 \pm 5.0$ & $29.9 \pm 6.1$ & $4.7 \pm 6.9$ & $25.2 \pm 6.8$ & $28.7 \pm 7.3$ & $3.5 \pm 9.9$ \\
\hline $\begin{array}{c}\text { External } \\
\text { Rotation (\%BM) }\end{array}$ & $18.2 \pm 3.9$ & $19.8 \pm 3.5$ & $1.6 \pm 3.8$ & $18.7 \pm 4.6$ & $19.6 \pm 3.7$ & $0.9 \pm 4.3$ \\
\hline $\begin{array}{c}\text { Internal } \\
\text { Rotation (\%BM) }\end{array}$ & $22.9 \pm 5.5$ & $26.9 \pm 6.4$ & $4.0 \pm 7.1$ & $23.3 \pm 5.5$ & $23.7 \pm 5.9$ & $0.4 \pm 7.1$ \\
\hline Abduction (\%BM) & $23.5 \pm 5.6$ & $25.2 \pm 5.1$ & $1.7 \pm 6.3$ & $25.5 \pm 6.7$ & $22.4 \pm 5.5$ & $-3.1 \pm 4.8$ \\
\hline Adduction (\%BM) & $30.8 \pm 8.1$ & $31.9 \pm 7.0$ & $1.1 \pm 7.4$ & $33.8 \pm 7.9$ & $34.4 \pm 7.0$ & $0.6 \pm 8.2$ \\
\hline Retraction (\%BM) & $18.3 \pm 4.6$ & $24.7 \pm 6.2$ & $6.4 \pm 4.9$ & $17.9 \pm 4.9$ & $24.0 \pm 5.4$ & $6.1 \pm 5.3$ \\
\hline $\begin{array}{l}\text { Retraction with } \\
\text { Downward } \\
\text { Rotation (\%BM) }\end{array}$ & $32.4 \pm 9.4$ & $35.4 \pm 10.3$ & $3.2 \pm 11.6$ & $32.9 \pm 6.3$ & $36.1 \pm 6.2$ & $3.2 \pm 7.0$ \\
\hline $\begin{array}{l}\text { Retraction with } \\
\text { Upward } \\
\text { Rotation (\%BM) }\end{array}$ & $16.9 \pm 4.0$ & $18.5 \pm 3.9$ & $1.6 \pm 4.1$ & $16.5 \pm 3.7$ & $17.5 \pm 3.6$ & $1.0 \pm 3.7$ \\
\hline
\end{tabular}


Table 3

\begin{tabular}{|c|c|c|c|c|c|c|}
\hline & \multicolumn{3}{|c|}{ Intervention } & \multicolumn{3}{|c|}{ Control } \\
\hline & Pre & Post & Change & Pre & Post & Change \\
\hline & Mean \pm SD & Mean \pm SD & Mean \pm SD & Mean \pm SD & Mean \pm SD & Mean \pm SD \\
\hline \multicolumn{7}{|l|}{ Internal/external rotation $\left({ }^{\circ}\right)$} \\
\hline $0^{\circ}$ humeral elevation & $22.2 \pm 6.8$ & $31.5 \pm 9.8$ & $9.3 \pm 11.9$ & $13.9 \pm 10.7$ & $23.9 \pm 12.5$ & $10.0 \pm 14.0$ \\
\hline $30^{\circ}$ humeral elevation & $21.5 \pm 6.7$ & $31.6 \pm 9.8$ & $10.1 \pm 10.7$ & $13.4 \pm 9.9$ & $23.7 \pm 12.6$ & $10.3 \pm 11.3$ \\
\hline $60^{\circ}$ humeral elevation & $20.5 \pm 7.2$ & $32.2 \pm 10.3$ & $11.8 \pm 11.8$ & $14.5 \pm 11.4$ & $24.2 \pm 13.1$ & $9.7 \pm 12.0$ \\
\hline $90^{\circ}$ humeral elevation & $21.6 \pm 8.4$ & $34.0 \pm 11.7$ & $12.4 \pm 12.1$ & $19.6 \pm 15.7$ & $27.4 \pm 13.4$ & $7.8 \pm 16.6$ \\
\hline $120^{\circ}$ humeral elevation & $24.2 \pm 12.4$ & $36.8 \pm 12.8$ & $12.6 \pm 15.1$ & $23.2 \pm 19.0$ & $34.8 \pm 17.5$ & $11.6 \pm 20.1$ \\
\hline \multicolumn{7}{|l|}{ Upward/downward rotation $\left({ }^{\circ}\right)$} \\
\hline $0^{\circ}$ humeral elevation & $7.7 \pm 7.6$ & $10.2 \pm 8.2$ & $2.5 \pm 8.5$ & $4.3 \pm 6.5$ & $4.8 \pm 6.6$ & $0.5 \pm 7.4$ \\
\hline $30^{\circ}$ humeral elevation & $9.4 \pm 7.1$ & $13.0 \pm 7.1$ & $2.6 \pm 7.6$ & $6.9 \pm 5.8$ & $8.9 \pm 5.9$ & $2.0 \pm 5.0$ \\
\hline $60^{\circ}$ humeral elevation & $20.1 \pm 6.4$ & $23.6 \pm 6.3$ & $3.5 \pm 6.7$ & $16.6 \pm 7.7$ & $19.1 \pm 6.5$ & $2.5 \pm 6.0$ \\
\hline $90^{\circ}$ humeral elevation & $33.5 \pm 9.1$ & $34.8 \pm 6.5$ & $1.3 \pm 8.9$ & $30.3 \pm 12.0$ & $31.0 \pm 8.2$ & $0.7 \pm 9.0$ \\
\hline $120^{\circ}$ humeral elevation & $34.1 \pm 9.7$ & $36.7 \pm 8.0$ & $2.6 \pm 10.8$ & $33.4 \pm 17.2$ & $38.0 \pm 13.6$ & $4.6 \pm 12.9$ \\
\hline \multicolumn{7}{|l|}{ Anterior/posterior tipping $\left({ }^{\circ}\right)$} \\
\hline $0^{\circ}$ humeral elevation & $7.9 \pm 4.8$ & $11.0 \pm 6.2$ & $3.1 \pm 6.7$ & $10.4 \pm 7.7$ & $12.1 \pm 4.8$ & $1.7 \pm 7.4$ \\
\hline $30^{\circ}$ humeral elevation & $7.2 \pm 5.4$ & $10.4 \pm 6.6$ & $3.2 \pm 7.4$ & $9.6 \pm 8.3$ & $10.8 \pm 5.5$ & $1.2 \pm 7.2$ \\
\hline $60^{\circ}$ humeral elevation & $3.6 \pm 6.6$ & $9.0 \pm 7.8$ & $5.4 \pm 9.0$ & $7.2 \pm 9.9$ & $8.5 \pm 6.7$ & $1.3 \pm 8.5$ \\
\hline $90^{\circ}$ humeral elevation & $-1.2 \pm 8.5$ & $5.0 \pm 9.7$ & $6.2 \pm 11.1$ & $4.0 \pm 9.6$ & $5.7 \pm 6.9$ & $1.7 \pm 9.8$ \\
\hline $120^{\circ}$ humeral elevation & $-0.5 \pm 9.1$ & $5.1 \pm 9.6$ & $5.6 \pm 10.8$ & $3.6 \pm 8.6$ & $4.9 \pm 7.3$ & $1.3 \pm 10.8$ \\
\hline \multicolumn{7}{|l|}{ Protraction/retraction $\left({ }^{\circ}\right)$} \\
\hline $0^{\circ}$ humeral elevation & $35.1 \pm 15.4$ & $23.5 \pm 7.8$ & $-11.6 \pm 19.6$ & $36.2 \pm 17.0$ & $28.7 \pm 8.5$ & $-7.5 \pm 14.0$ \\
\hline $30^{\circ}$ humeral elevation & $35.7 \pm 15.0$ & $24.0 \pm 7.8$ & $-11.7 \pm 19.5$ & $36.8 \pm 17.8$ & $28.5 \pm 8.4$ & $-8.1 \pm 15.7$ \\
\hline $60^{\circ}$ humeral elevation & $37.8 \pm 14.7$ & $26.4 \pm 7.5$ & $-11.4 \pm 19.7$ & $36.1 \pm 20.2$ & $30.5 \pm 8.3$ & $-5.6 \pm 17.4$ \\
\hline $90^{\circ}$ humeral elevation & $40.6 \pm 14.7$ & $30.0 \pm 7.7$ & $-10.6 \pm 20.0$ & $33.2 \pm 17.4$ & $33.5 \pm 8.0$ & $0.3 \pm 14.8$ \\
\hline $120^{\circ}$ humeral elevation & $40.9 \pm 14.9$ & $30.0 \pm 8.2$ & $-10.9 \pm 20.0$ & $33.9 \pm 17.7$ & $35.5 \pm 9.4$ & $1.6 \pm 16.1$ \\
\hline \multicolumn{7}{|l|}{ Elevation/depression $\left({ }^{\circ}\right)$} \\
\hline $0^{\circ}$ humeral elevation & $4.5 \pm 6.1$ & $8.4 \pm 7.5$ & $3.9 \pm 6.6$ & $2.2 \pm 9.1$ & $6.3 \pm 6.1$ & $4.1 \pm 8.4$ \\
\hline $30^{\circ}$ humeral elevation & $5.3 \pm 5.9$ & $9.5 \pm 7.1$ & $4.2 \pm 5.9$ & $4.1 \pm 8.3$ & $7.5 \pm 6.7$ & $3.4 \pm 7.6$ \\
\hline $60^{\circ}$ humeral elevation & $9.8 \pm 5.8$ & $14.7 \pm 6.9$ & $4.9 \pm 5.5$ & $14.7 \pm 9.8$ & $12.3 \pm 7.2$ & $-2.4 \pm 10.5$ \\
\hline $90^{\circ}$ humeral elevation & $15.7 \pm 6.3$ & $21.0 \pm 7.4$ & $5.3 \pm 6.8$ & $23.2 \pm 14.2$ & $18.4 \pm 7.9$ & $-4.8 \pm 15.7$ \\
\hline $120^{\circ}$ humeral elevation & $16.0 \pm 6.5$ & $22.0 \pm 7.7$ & $6.0 \pm 6.5$ & $25.0 \pm 14.0$ & $22.0 \pm 9.5$ & $-3.0 \pm 15.6$ \\
\hline
\end{tabular}


Table 4

\begin{tabular}{ccccccc}
\hline & $\begin{array}{c}\text { Pre } \\
\text { Mean } \pm \text { SD }\end{array}$ & $\begin{array}{c}\text { Intervention } \\
\text { Mest }\end{array}$ & $\begin{array}{c}\text { Change } \\
\text { Mean } \pm \text { SD }\end{array}$ & $\begin{array}{c}\text { Pre } \\
\text { Mean } \pm \text { SD }\end{array}$ & $\begin{array}{c}\text { Control } \\
\text { Mean } \pm \text { SD }\end{array}$ & $\begin{array}{c}\text { Change } \\
\text { Mean } \pm \text { SD }\end{array}$ \\
\hline SANE & $89.6 \pm 16.6$ & $90.3 \pm 10.5$ & $0.7 \pm 15.0$ & $92.0 \pm 9.5$ & $92.1 \pm 8.7$ & $0.1 \pm 8.8$ \\
$\begin{array}{c}\text { DASH } \\
\text { Composite } \\
\text { Score }\end{array}$ & $6.3 \pm 11.8$ & $10.0 \pm 15.6$ & $3.7 \pm 14.7$ & $6.3 \pm 13.4$ & $4.5 \pm 7.4$ & $-1.8 \pm 9.7$
\end{tabular}




\section{REFERENCES}

Allegrucci, M., S. L. Whitney, et al. (1994). "Clinical implications of secondary impingement of the shoulder in freestyle swimmers." J Orthop Sports Phys Ther 20(6): 307-18.

Andrews, J. R., K. E. Wilk, et al. (2008). The Athlete's Shoulder, A Churchhill Linvingstone Title.

Arroyo, J. S., S. J. Hershon, et al. (1997). "Special considerations in the athletic throwing shoulder." Orthop Clin North Am 28(1): 69-78.

Bak, K. and P. Fauno (1997). "Clinical findings in competitive swimmers with shoulder pain." Am J Sports Med 25(2): 254-60.

Bak, K. and S. P. Magnusson (1997). "Shoulder strength and range of motion in symptomatic and pain-free elite swimmers." Am J Sports Med 25(4): 454-9.

Ballantyne, B. T., S. J. O'Hare, et al. (1993). "Electromyographic activity of selected shoulder muscles in commonly used therapeutic exercises." Phys Ther 73(10): 668-77; discussion 677-82.

Bassett, R. W., A. O. Browne, et al. (1990). "Glenohumeral muscle force and moment mechanics in a position of shoulder instability." J Biomech 23(5): 405-15.

Beach, M. L., S. L. Whitney, et al. (1992). "Relationship of shoulder flexibility, strength, and endurance to shoulder pain in competitive swimmers." J Orthop Sports Phys Ther 16(6): 262-8.

Bechtol, C. O. (1980). "Biomechanics of the shoulder." Clin Orthop Relat Res(146): 3741. 
Belzer, J. P. and R. C. Durkin (1996). "Common disorders of the shoulder." Prim Care 23(2): 365-88.

Bigliani, L. U., D. S. Morrison, et al. (1986). "The morphology of the acromion and its relationship to rotator cuff tears." Orthop. Trans. 10.

Bongers, P. M. (2001). "The cost of shoulder pain at work." BMJ 322(7278): 64-65.

Borich, M. R., J. M. Bright, et al. (2006). "Scapular angular positioning at end range internal rotation in cases of glenohumeral internal rotation deficit." J Orthop Sports Phys Ther 36(12): 926-34.

Borstad, J. D. and P. M. Ludewig (2005). "The effect of long versus short pectoralis minor resting length on scapular kinematics in healthy individuals." J Orthop Sports Phys Ther 35(4): 227-38.

Borstad, J. D. and P. M. Ludewig (2006). "Comparison of three stretches for the pectoralis minor muscle." J Shoulder Elbow Surg 15(3): 324-30.

Burkhart, S. S., C. D. Morgan, et al. (2003). "The disabled throwing shoulder: spectrum of pathology Part I: pathoanatomy and biomechanics." Arthroscopy 19(4): 40420.

Chang, W. K. (2004). "Shoulder impingement syndrome." Phys Med Rehabil Clin N Am 15(2): 493-510.

Cissik, J. (2002). "Basic Principles of Strength Training and Conditioning." NSCA's Performance Training Journal 1(4): 7-11.

Cohen, R. B. and G. R. Williams, Jr. (1998). "Impingement syndrome and rotator cuff disease as repetitive motion disorders." Clin Orthop Relat Res(351): 95-101.

Cools, A. M., G. A. Declercq, et al. (2007). "Trapezius activity and intramuscular balance during isokinetic exercise in overhead athletes with impingement symptoms." Scand J Med Sci Sports 17(1): 25-33.

Cools, A. M., V. Dewitte, et al. (2007). "Rehabilitation of scapular muscle balance: which exercises to prescribe?" Am J Sports Med 35(10): 1744-51. 
Cools, A. M., E. E. Witvrouw, et al. (2003). "Scapular muscle recruitment patterns: trapezius muscle latency with and without impingement symptoms." Am J Sports Med 31(4): 542-9.

Cordasco, F. A., I. N. Wolfe, et al. (1996). "An electromyographic analysis of the shoulder during a medicine ball rehabilitation program." Am J Sports Med 24(3): $386-92$.

Costill, D. L., W. J. Fink, et al. (1985). "Metabolic characteristics of skeletal muscle during detraining from competitive swimming." Med Sci Sports Exerc 17(3): 33943.

Costill, D. L., J. Kovaleski, et al. (1985). "Energy expenditure during front crawl swimming: predicting success in middle-distance events." Int J Sports Med 6(5): 266-70.

Crockett, H. C., L. B. Gross, et al. (2002). "Osseous adaptation and range of motion at the glenohumeral joint in professional baseball pitchers." Am J Sports Med 30(1): 206.

Culham, E. and M. Peat (1993). "Functional anatomy of the shoulder complex." J Orthop Sports Phys Ther 18(1): 342-50.

Decker, M. J., R. A. Hintermeister, et al. (1999). "Serratus anterior muscle activity during selected rehabilitation exercises." Am J Sports Med 27(6): 784-91.

Deutsch, A., D. W. Altchek, et al. (1996). "Radiologic measurement of superior displacement of the humeral head in the impingement syndrome." J Shoulder Elbow Surg 5(3): 186-93.

Dines, J. S., J. B. Frank, et al. (2009). "Glenohumeral internal rotation deficits in baseball players with ulnar collateral ligament insufficiency." Am J Sports Med 37(3): 566-70.

Donatelli, R. A. (2004). Physical Therapy of the Shoulder, Churchill Livingstone.

Ekstrom, R. A., R. A. Donatelli, et al. (2003). "Surface electromyographic analysis of exercises for the trapezius and serratus anterior muscles." J Orthop Sports Phys Ther 33(5): 247-58. 
Endo, K., T. Ikata, et al. (2001). "Radiographic assessment of scapular rotational tilt in chronic shoulder impingement syndrome." J Orthop Sci 6(1): 3-10.

Falla, D., D. Farina, et al. (2007). "Experimental muscle pain results in reorganization of coordination among trapezius muscle subdivisions during repetitive shoulder flexion." Exp Brain Res 178(3): 385-93.

Farina, D., L. Arendt-Nielsen, et al. (2004). "Effect of experimental muscle pain on motor unit firing rate and conduction velocity." J Neurophysiol 91(3): 1250-9.

Finley, M. A. and R. Y. Lee (2003). "Effect of sitting posture on 3-dimensional scapular kinematics measured by skin-mounted electromagnetic tracking sensors." Arch Phys Med Rehabil 84(4): 563-8.

Fu, F. H., C. D. Harner, et al. (1991). "Shoulder impingement syndrome. A critical review." Clin Orthop Relat Res(269): 162-73.

Graichen, H., H. Bonel, et al. (1999). "Three-dimensional analysis of the width of the subacromial space in healthy subjects and patients with impingement syndrome." AJR Am J Roentgenol 172(4): 1081-6.

Greenfield, B., P. A. Catlin, et al. (1995). "Posture in patients with shoulder overuse injuries and healthy individuals." J Orthop Sports Phys Ther 21(5): 287-95.

Grossman, M. G., J. E. Tibone, et al. (2005). "A cadaveric model of the throwing shoulder: a possible etiology of superior labrum anterior-to-posterior lesions." J Bone Joint Surg Am 87(4): 824-31.

Hardy, D. C., J. B. Vogler, 3rd, et al. (1986). "The shoulder impingement syndrome: prevalence of radiographic findings and correlation with response to therapy." AJR Am J Roentgenol 147(3): 557-61.

Harryman, D. T., 2nd, J. A. Sidles, et al. (1990). "Translation of the humeral head on the glenoid with passive glenohumeral motion." J Bone Joint Surg Am 72(9): 133443.

Hawkins, R. J., G. W. Misamore, et al. (1985). "Surgery for full-thickness rotator-cuff tears." J Bone Joint Surg Am 67(9): 1349-55. 
Hawley, J. A. and M. M. Williams (1991). "Relationship between upper body anaerobic power and freestyle swimming performance." Int J Sports Med 12(1): 1-5.

Hayes, K., J. R. Walton, et al. (2002). "Reliability of 3 methods for assessing shoulder strength." J Shoulder Elbow Surg 11(1): 33-9.

Hebert, L. J., H. Moffet, et al. (2002). "Scapular behavior in shoulder impingement syndrome." Arch Phys Med Rehabil 83(1): 60-9.

Hernigou, P., F. Duparc, et al. (2002). "Determining humeral retroversion with computed tomography." J Bone Joint Surg Am 84-A(10): 1753-62.

Hintermeister, R. A., G. W. Lange, et al. (1998). "Electromyographic activity and applied load during shoulder rehabilitation exercises using elastic resistance." Am J Sports Med 26(2): 210-20.

Huisstede, B. M., A. Feleus, et al. (2009). "Is the disability of arm, shoulder, and hand questionnaire (DASH) also valid and responsive." Spine 34(4): E130-8.

Illyes, A. and R. M. Kiss (2006). "Kinematic and muscle activity characteristics of multidirectional shoulder joint instability during elevation." Knee Surg Sports Traumatol Arthrosc 14(7): 673-85.

Inman, V. T., J. B. Saunders, et al. (1996). "Observations of the function of the shoulder joint. 1944." Clin Orthop Relat Res(330): 3-12.

Izumi, T., M. Aoki, et al. (2008). "Stretching positions for the posterior capsule of the glenohumeral joint: strain measurement using cadaver specimens." Am J Sports Med 36(10): 2014-22.

Johnson, D. (1988). "In Swimming, shoulder the burden

." Sportcare Fitness May-June: 24-30.

Johnson, J. N., J. Gauvin, et al. (2003). "Swimming Biomechanics and Injury Prevention." Physcian and Sports Medicine 31(1): 41-48.

Karduna, A. R., P. J. Kerner, et al. (2005). "Contact forces in the subacromial space: effects of scapular orientation." J Shoulder Elbow Surg 14(4): 393-9. 
Karduna, A. R., P. W. McClure, et al. (2000). "Scapular Kinematics: Effects of altering the Euler Angle of sequence of rotations." J Biomech 33: 1063-1068.

Karduna, A. R., P. W. McClure, et al. (2001). "Dynamic measurements of threedimensional scapular kinematics: a validation study." J Biomech Eng 123(2): 18490 .

Kebaetse, M., P. McClure, et al. (1999). "Thoracic position effect on shoulder range of motion, strength, and three-dimensional scapular kinematics." Arch Phys Med Rehabil 80(8): 945-50.

Kendall, F. P., E. K. McCreary, et al. (2005). Muscle Testing and Function, with Posture and Pain. Baltimore, Lippincott Williams \& Wilkins.

Kibler, W. B. (1998). "The role of the scapula in athletic shoulder function." Am J Sports Med 26(2): 325-37.

Kibler, W. B. and J. McMullen (2003). "Scapular dyskinesis and its relation to shoulder pain." J Am Acad Orthop Surg 11(2): 142-51.

Kibler, W. B., A. D. Sciascia, et al. (2008). "Electromyographic analysis of specific exercises for scapular control in early phases of shoulder rehabilitation." Am J Sports Med 36(9): 1789-98.

Kluemper, M., T. L. Uhl, et al. (2006). "Effect of Stretching and Strengthening Shoulder Muscles on Forward Shoulder Posture in Competitive Swimmers." J Sport Rehabil 15: 58-70.

Ko, J. Y., C. C. Huang, et al. (2006). "Pathogenesis of partial tear of the rotator cuff: a clinical and pathologic study." J Shoulder Elbow Surg 15(3): 271-8.

Krupp, R. J., M. A. Kevern, et al. (2009). "Long head of the biceps tendon pain: differential diagnosis and treatment." J Orthop Sports Phys Ther 39(2): 55-70.

Kumar, V. P., K. Satku, et al. (1989). "The role of the long head of biceps brachii in the stabilization of the head of the humerus." Clin Orthop Relat Res(244): 172-5. 
Laudner, K. G., R. C. Sipes, et al. (2008). "The acute effects of sleeper stretches on shoulder range of motion." J Athl Train 43(4): 359-63.

Layton, J., C. A. Thigpen, et al. (2005). A Comparison Between Swimmers and NonSwimmers on Posture, Range of Motion, Strength, and Scapular Motion. National Athletic Training Association Annual Meeting. Indianapolis.

Leggin, B. G., R. M. Neuman, et al. (1996). "Intrarater and interrater reliability of three isometric dynamometers in assessing shoulder strength." J Shoulder Elbow Surg 5(1): 18-24.

Lewis, J. S., A. Green, et al. (2005). "Subacromial impingement syndrome: the role of posture and muscle imbalance." J Shoulder Elbow Surg 14(4): 385-92.

Lin, J. J., W. P. Hanten, et al. (2005). "Functional activity characteristics of individuals with shoulder dysfunctions." J Electromyogr Kinesiol 15(6): 576-86.

Lin, J. J., H. K. Lim, et al. (2006). "Effect of shoulder tightness on glenohumeral translation, scapular kinematics, and scapulohumeral rhythm in subjects with stiff shoulders." J Orthop Res 24(5): 1044-51.

Lintner, D., M. Mayol, et al. (2007). "Glenohumeral internal rotation deficits in professional pitchers enrolled in an internal rotation stretching program." Am J Sports Med 35(4): 617-21.

Ludewig, P. M., S. A. Behrens, et al. (2004). "Three-dimensional clavicular motion during arm elevation: reliability and descriptive data." J Orthop Sports Phys Ther 34(3): 140-9.

Ludewig, P. M. and T. M. Cook (2000). "Alterations in shoulder kinematics and associated muscle activity in people with symptoms of shoulder impingement." Phys Ther 80(3): 276-91.

Ludewig, P. M., T. M. Cook, et al. (1996). "Three-dimensional scapular orientation and muscle activity at selected positions of humeral elevation." J Orthop Sports Phys Ther 24(2): 57-65. 
Ludewig, P. M., M. S. Hoff, et al. (2004). "Relative balance of serratus anterior and upper trapezius muscle activity during push-up exercises." Am J Sports Med 32(2): 484-93.

Ludewig, P. M. and J. E. Reynolds (2009). "The association of scapular kinematics and glenohumeral joint pathologies." J Orthop Sports Phys Ther 39(2): 90-104.

Lukasiewicz, A. C., P. McClure, et al. (1999). "Comparison of 3-dimensional scapular position and orientation between subjects with and without shoulder impingement." J Orthop Sports Phys Ther 29(10): 574-83; discussion 584-6.

Lysholm, J. and J. Gillquist (1982). "Evaluation of knee ligament surgery results with special emphasis on use of a scoring scale." Am J Sports Med 10(3): 150-4.

MacGillivray, J. D., S. Fealy, et al. (1998). "Multiplanar analysis of acromion morphology." Am J Sports Med 26(6): 836-40.

McClure, P., J. Balaicuis, et al. (2007). "A randomized controlled comparison of stretching procedures for posterior shoulder tightness." J Orthop Sports Phys Ther 37(3): 108-14.

McClure, P. W., J. Bialker, et al. (2004). "Shoulder function and 3-dimensional kinematics in people with shoulder impingement syndrome before and after a 6week exercise program." Phys Ther 84(9): 832-48.

McClure, P. W., L. A. Michener, et al. (2006). "Shoulder function and 3-dimensional scapular kinematics in people with and without shoulder impingement syndrome." Phys Ther 86(8): 1075-90.

McFarland, E. G. and M. Wasik (1996). "Injuries in female collegiate swimmers due to swimming and cross training." Clin J Sport Med 6(3): 178-82.

McLeod, I. (2010). Swimming Anatomy. Champaign, Human Kinetics.

McMaster, W. C. (1994). "Assessment of the rotator cuff and a remedial exercise program for the aquatic athlete." Med Sci Sports Exerc 39: 213-217. 
McMaster, W. C. (1999). "Shoulder injuries in competitive swimmers." Clin Sports Med 18(2): 349-59, vii.

McMaster, W. C., A. Roberts, et al. (1998). "A correlation between shoulder laxity and interfering pain in competitive swimmers." Am J Sports Med 26(1): 83-6.

Meskers, C. G., H. M. Vermeulen, et al. (1998). "3D shoulder position measurements using a six-degree-of-freedom electromagnetic tracking device." Clin Biomech (Bristol, Avon) 13(4-5): 280-292.

Meyer, A. (1931). "The minuter anatomy of attrition lesions." J Bone Joint Surg Am 13.

Michener, L. A., P. W. McClure, et al. (2003). "Anatomical and biomechanical mechanisms of subacromial impingement syndrome." Clin Biomech (Bristol, Avon) 18(5): 369-79.

Monod, H. (1985). "Contractility of muscle during prolonged static and repetitive dynamic activity." Ergonomics 28(1): 81-9.

Moore, K. and A. Dalley (2006). Clinically Oriented Anatomy. Philadelphia, Lipincott Williams \& Wilkins.

Moraes, G. F., C. D. Faria, et al. (2008). "Scapular muscle recruitment patterns and isokinetic strength ratios of the shoulder rotator muscles in individuals with and without impingement syndrome." J Shoulder Elbow Surg 17(1 Suppl): 48S-53S.

Moseley, J. B., Jr., F. W. Jobe, et al. (1992). "EMG analysis of the scapular muscles during a shoulder rehabilitation program." Am J Sports Med 20(2): 128-34.

Myers, J., J. Jolly, et al. (2006). "Reliability and Precision of in Vivo Scapular Kinematic Measurements Using an Electromagnetic Tracking Device." J Sport Rehabil(15): $125-43$.

Myers, J. B., J. H. Hwang, et al. (2009). "Rotator cuff coactivation ratios in participants with subacromial impingement syndrome." J Sci Med Sport 12(6): 603-8.

Myers, J. B., K. G. Laudner, et al. (2005). "Scapular position and orientation in throwing athletes." Am J Sports Med 33(2): 263-71. 
Myers, J. B., K. G. Laudner, et al. (2006). "Glenohumeral range of motion deficits and posterior shoulder tightness in throwers with pathologic internal impingement." Am J Sports Med 34(3): 385-91.

Myers, J. B., S. Oyama, et al. ((In Press)). "Influence of humeral torsion on interpretation of posterior shoulder tightness measures in overhead athletes." Clin J Sport Med.

Myers, J. B., M. R. Pasquale, et al. (2005). "On-the-Field Resistance-Tubing Exercises for Throwers: An Electromyographic Analysis." J Athl Train 40(1): 15-22.

Neer, C. S., 2nd (1983). "Impingement lesions." Clin Orthop Relat Res(173): 70-7.

Neer, C. S., 2nd (2005). "Anterior acromioplasty for the chronic impingement syndrome in the shoulder. 1972." J Bone Joint Surg Am 87(6): 1399.

Neufer, P. D. (1989). "The effect of detraining and reduced training on the physiological adaptations to aerobic exercise training." Sports Med 8(5): 302-20.

Opsha, O., A. Malik, et al. (2008). "MRI of the rotator cuff and internal derangement." Eur J Radiol 68(1): 36-56.

Osbahr, D. C., D. L. Cannon, et al. (2002). "Retroversion of the humerus in the throwing shoulder of college baseball pitchers." Am J Sports Med 30(3): 347-53.

Oyama, S. and J. B. Myers (In Review). "Effectiveness of Scapular Stabilizing Musculature During Common Rehabilitation Exercises."

Pagnani, M. J., X. H. Deng, et al. (1996). "Role of the long head of the biceps brachii in glenohumeral stability: a biomechanical study in cadavera." J Shoulder Elbow Surg 5(4): 255-62.

Peat, M. and R. E. Grahame (1977). "Electromyographic analysis of soft tissue lesions affecting shoulder function." Am J Phys Med 56(5): 223-40.

Pezzullo, D. J., S. Karas, et al. (1995). "Functional Plyometric Exercises for the Throwing Athlete." J Athl Train 30(1): 22-26. 
Pieper, H. G. (1998). "Humeral torsion in the throwing arm of handball players." Am J Sports Med 26(2): 247-53.

Pink, M., J. Perry, et al. (1991). "The normal shoulder during freestyle swimming. An electromyographic and cinematographic analysis of twelve muscles." Am J Sports Med 19(6): 569-76.

Pink, M. M. and J. E. Tibone (2000). "The painful shoulder in the swimming athlete." Orthop Clin North Am 31(2): 247-61.

Ramsi, M., K. Swanik, et al. (2004). "Shoulder-Rotator Strength of High School Swimmers Over the Course of a Competitive Season." J Sport Rehabil 13(1): 918.

Reagan, K. M., K. Meister, et al. (2002). "Humeral retroversion and its relationship to glenohumeral rotation in the shoulder of college baseball players." Am J Sports Med 30(3): 354-60.

Reinold, M. M., L. C. Macrina, et al. (2007). "Electromyographic analysis of the supraspinatus and deltoid muscles during 3 common rehabilitation exercises." $\underline{\mathrm{J}}$ Athl Train 42(4): 464-9.

Richardson, A. R. (1986). "The biomechanics of swimming: the shoulder and knee." $\underline{\text { Clin }}$ Sports Med 5(1): 103-13.

Rodosky, M. W., C. D. Harner, et al. (1994). "The role of the long head of the biceps muscle and superior glenoid labrum in anterior stability of the shoulder." $\mathrm{Am} \mathrm{J}$ Sports Med 22(1): 121-30.

Sabick, M. B., Y. K. Kim, et al. (2005). "Biomechanics of the shoulder in youth baseball pitchers: implications for the development of proximal humeral epiphysiolysis and humeral retrotorsion." Am J Sports Med 33(11): 1716-22.

Sabick, M. B., M. R. Torry, et al. (2004). "Humeral torque in professional baseball pitchers." Am J Sports Med 32(4): 892-8.

Santos, M. J., W. D. Belangero, et al. (2007). "The effect of joint instability on latency and recruitment order of the shoulder muscles." J Electromyogr Kinesiol 17(2): $167-75$. 
Scovazzo, M. L., A. Browne, et al. (1991). "The painful shoulder during freestyle swimming. An electromyographic cinematographic analysis of twelve muscles." Am J Sports Med 19(6): 577-82.

Solem-Bertoft, E., K. A. Thuomas, et al. (1993). "The influence of scapular retraction and protraction on the width of the subacromial space. An MRI study." Clin Orthop Relat Res(296): 99-103.

Solway, S., D. Beaton, et al. (2002). The DASH Outcome Measure User's Manual. Toronto, Institute for Work \& Health.

Soslowsky, L. J., S. Thomopoulos, et al. (2002). "Rotator cuff tendinosis in an animal model: role of extrinsic and overuse factors." Ann Biomed Eng 30(8): 1057-63.

Stewart, A. M. and W. G. Hopkins (2000). "Seasonal training and performance of competitive swimmers." J Sports Sci 18(11): 873-84.

Stocker, D., M. Pink, et al. (1995). "Comparison of shoulder injury in collegiate- and master's-level swimmers." Clin J Sport Med 5(1): 4-8.

Stokdijk, M., J. Nagels, et al. (2000). "The glenohumeral joint rotation centre in vivo." $\underline{\mathrm{J}}$ Biomech 33(12): 1629-36.

Stratford, P. W. and B. E. Balsor (1994). "A comparison of make and break tests using a hand-held dynamometer and the Kin-Com." J Orthop Sports Phys Ther 19(1): 2832.

Su, K. P., M. P. Johnson, et al. (2004). "Scapular rotation in swimmers with and without impingement syndrome: practice effects." Med Sci Sports Exerc 36(7): 1117-23.

Swanik, K., C. Swanik, et al. (2002). "The effect of functional training on the incidence of shoulder pain and strength in intercollegiate swimmers." J Sport Rehabil 11(2): 142-154.

Swanik, K. A., S. M. Lephart, et al. (2002). "The effects of shoulder plyometric training on proprioception and selected muscle performance characteristics." J Shoulder Elbow Surg 11(6): 579-86. 
Tegner, Y. and J. Lysholm (1985). "Rating systems in the evaluation of knee ligament injuries." Clin Orthop Relat Res(198): 43-9.

Thigpen, C. A., M. T. Gross, et al. (2005). "The repeatability of scapular rotations across three planes of humeral elevation." Res Sports Med 13(3): 181-98.

Thigpen, C. A., D. A. Padua, et al. (2006). "Scapular kinematics during supraspinatus rehabilitation exercise: a comparison of full-can versus empty-can techniques." Am J Sports Med 34(4): 644-52.

Tovin, B. J. (2006). "Prevention and Treatment of Swimmer's Shoulder." North American Journal of Sports Physical Therapy 1(4): 166-175.

Troup, J. P. (1999). "The physiology and biomechanics of competitive swimming." Clin Sports Med 18(2): 267-85.

Tsai, N. T., P. W. McClure, et al. (2003). "Effects of Muscle Fatigue on 3-Dimensional Scapular Kinematics." Arch Phys Med Rehabil 84: 1000-1005.

Tuite, M. J., J. R. Turnbull, et al. (1998). "Anterior versus posterior, and rim-rent rotator cuff tears: prevalence and MR sensitivity." Skeletal Radiol 27(5): 237-43.

Tyler, T. F., A. Cuoco, et al. (2009). "The effect of scapular-retractor fatigue on external and internal rotation in patients with internal impingement." J Sport Rehabil 18(2): 229-39.

Tyler, T. F., S. J. Nicholas, et al. (2000). "Quantification of posterior capsule tightness and motion loss in patients with shoulder impingement." Am J Sports Med 28(5): $668-73$.

Urwin, M., D. Symmons, et al. (1998). "Estimating the burden of muscuolskeletal disorders in the community: The comparative prevalence of symptoms at different antatomical sites and the relation to social deprivation." Ann Rheum Dis 57(11): $649-655$

Voight, M. L. and B. C. Thomson (2000). "The Role of the Scapula in the Rehabilitation of Shoulder Injuries." J Athl Train 35(3): 364-372. 
von Eisenhart-Rothe, R., F. A. Matsen, 3rd, et al. (2005). "Pathomechanics in atraumatic shoulder instability: scapular positioning correlates with humeral head centering." Clin Orthop Relat Res(433): 82-9.

Wadsworth, D. J. and J. E. Bullock-Saxton (1997). "Recruitment patterns of the scapular rotator muscles in freestyle swimmers with subacromial impingement." Int J Sports Med 18(8): 618-24.

Wang, C. H., P. McClure, et al. (1999). "Stretching and strengthening exercises: their effect on three-dimensional scapular kinematics." Arch Phys Med Rehabil 80(8): 923-9.

Warner, J. J., A. A. Allen, et al. (1997). "Arthroscopic release of postoperative capsular contracture of the shoulder." J Bone Joint Surg Am 79(8): 1151-8.

Warner, J. J. and G. M. Beim (1997). "Combined Bankart and HAGL lesion associated with anterior shoulder instability." Arthroscopy 13(6): 749-52.

Warner, J. J., L. J. Micheli, et al. (1990). "Patterns of flexibility, laxity, and strength in normal shoulders and shoulders with instability and impingement." Am J Sports Med 18(4): 366-75.

Weldon, E. J., 3rd and A. B. Richardson (2001). "Upper extremity overuse injuries in swimming. A discussion of swimmer's shoulder." Clin Sports Med 20(3): 423-38.

Wilk, K. E., P. Obma, et al. (2009). "Shoulder injuries in the overhead athlete." J Orthop Sports Phys Ther 39(2): 38-54.

Williams, G. N., T. J. Gangel, et al. (1999). "Comparison of the Single Assessment Numeric Evaluation method and two shoulder rating scales. Outcomes measures after shoulder surgery." Am J Sports Med 27(2): 214-21.

Williams, G. N., D. C. Taylor, et al. (2000). "Comparison of the single assessment numeric evaluation method and the Lysholm score." Clin Orthop Relat Res(373): $184-92$.

Worland, R. L., D. Lee, et al. (2003). "Correlation of age, acromial morphology, and rotator cuff tear pathology diagnosed by ultrasound in asymptomatic patients." $\underline{\mathrm{J}}$ South Orthop Assoc 12(1): 23-6. 
Wu, G., F. C. van der Helm, et al. (2005). "ISB recommendation on definitions of joint coordinate systems of various joints for the reporting of human joint motion--Part II: shoulder, elbow, wrist and hand." J Biomech 38(5): 981-992.

Yanai, T. and J. G. Hay (2000). "Shoulder impingement in front-crawl swimming: II. Analysis of stroking technique." Med Sci Sports Exerc 32(1): 30-40.

Yanai, T., J. G. Hay, et al. (2000). "Shoulder impingement in front-crawl swimming: I. A method to identify impingement." Med Sci Sports Exerc 32(1): 21-9.

Zemek, M. J. and D. J. Magee (1996). "Comparison of glenohumeral joint laxity in elite and recreational swimmers." Clin J Sport Med 6(1): 40-7. 